\title{
A Game-theoretic Analysis of Link Adaptation in Cellular Radio Networks
}

\author{
Samir V. Ginde
}

Thesis submitted to the faculty of the Virginia Polytechnic Institute and State University in partial fulfillment of the requirements for the degree of

\author{
Master of Science \\ In \\ Electrical Engineering
}

Dr. R. Michael Buehrer, Chair

Dr. Scott F. Midkiff

Dr. Robert P. Gilles

May 7, 2004

Blacksburg, Virginia

Keywords: Radio resource management, Power control, Link adaptation, Game theory, Nash equilibrium 


\title{
A Game-theoretic Analysis of Link Adaptation in Cellular Radio Networks
}

\author{
Samir V. Ginde
}

\begin{abstract}
In recent years, game theory has emerged as a promising approach to solving the power control problem in wireless networks. This thesis extends the reach of game-theoretic analysis to embrace link adaptation, thereby constituting a generalization of the power control problem. A realistic and natural problem formulation is attempted, wherein transmitter power and a discrete-valued Adaptable Link Parameter (ALP), e.g. code rate, constitute the action set of a player in this game. The dual goals of maximizing throughput and minimizing power consumption are reflected in the utility function selection, which uses the accurate sigmoid model for approximating throughput. The discrete action space makes it difficult to verify the existence of a Nash Equilibrium (NE) in this game using standard techniques. To circumvent this limitation, a heuristic algorithm is proposed. This algorithm is analytically shown to always converge to a NE. The subsequent results probe its validity and sensitivity. Favorable comparisons are drawn between these game-theoretic results and those arising from parallel systems techniques. A linear programming system optimization that exploits properties of the dominant eigenvalue of the system gain matrix is also presented in a comparative context.
\end{abstract}




\section{Acknowledgements}

This thesis is by no means entirely the work of a single person. I would, therefore, like to express my sincerest gratitude to several people, each of whom has had an impact on my life as a graduate at Virginia Tech.

I would first like to acknowledge the contribution of my advisor, Dr. Mike Buehrer, whose guidance, support, insight and leadership is reflected in all my work; my thesis committee, Dr. Gilles and Dr. Midkiff, for their suggestions; our research sponsor, the Motorola University Partnership in Research, who provided the all-important financial backing; and Jody Neel, my friend and collaborator at MPRG, whose hard work and enterprise brought the Motorola Project amongst others, to MPRG.

I would like to thank several people who perhaps will never read this thesis, but have yet contributed to it! Words are insufficient to express my gratitude towards my parents, sister and grandmother, all in India, who are and always have been a source of infinite love and support for me. I also thank my relatives in the US and my host family in Blacksburg for making the States "home" for me; my flat mates for making the small moments fun, Kuntal for "always" being there, and numerous friends who wish the best for me.

MPRG is a truly great research group! I thank ALL the students, faculty and staff who have made my life for the last two years exciting and fulfilling. I would like to especially mention James Hicks for several stimulating discussions, Dr. Allen MacKenzie for checking a key proof, Dr. Jeff Reed for his encouragement and generosity, and the members of the Game Theory Group at MPRG for their collective inputs.

The people behind the scenes have contributed no less - the ECE department, the Graduate School, the Cranwell International Center, and Virginia Tech; their efficient and tireless contributions go a long way in creating a pleasant and successful student experience. I thank them all.

Finally, I thank God, for giving me the strength and self-belief to accomplish and enjoy this challenge, from which I believe I have emerged as a better human being. 


\section{Table of Contents}

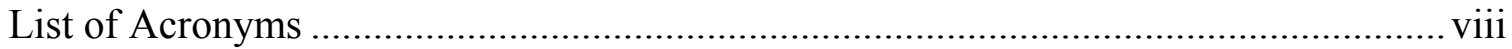

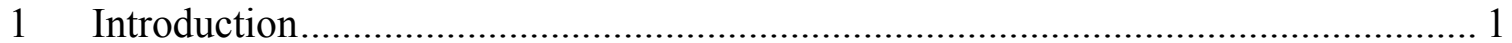

$1.1 \quad$ Third Generation (3G) Wireless Communications ............................................ 1

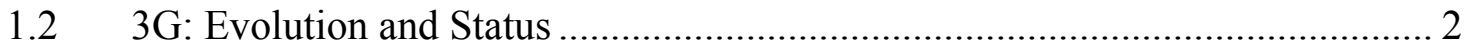

1.3 Radio Resource Management (RRM) for 3G Networks.................................... 3

2 Power Control in RRM ................................................................................ 7

$2.1 \quad$ Introduction to Power Control ....................................................................... 7

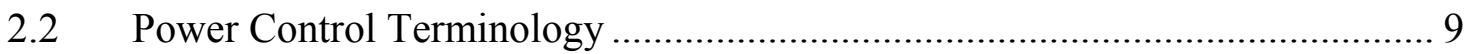

2.2.1 CDMA Power Control .................................................................... 10

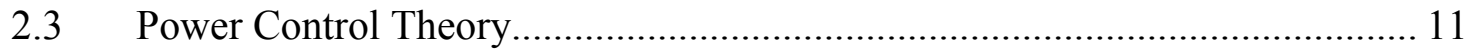

$2.4 \quad$ Multi-rate Power Control …………........................................................... 14

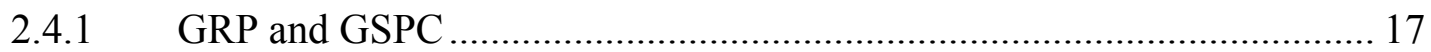

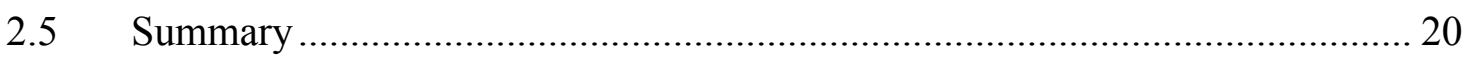

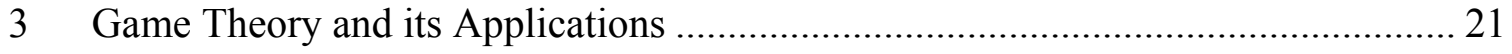

$3.1 \quad$ Introduction to Game Theory........................................................................ 21

3.2 Applications of Game Theory .................................................................. 22

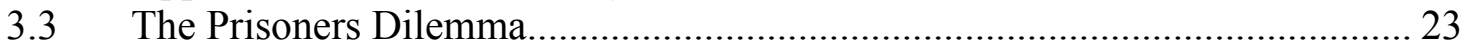

3.4 A Brief History of Modern Game Theory …………....................................... 23

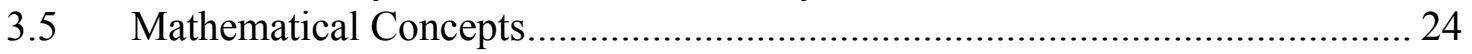

3.6 Application of Game Theory to Power Control............................................. 30

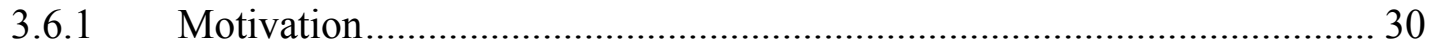

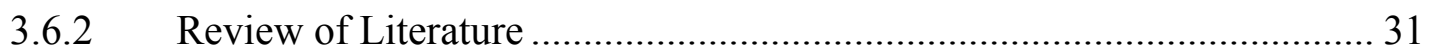

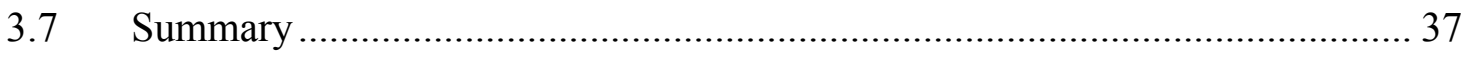

4 A Game-theoretic Approach to Link Adaptation................................................... 38

$4.1 \quad$ Link Adaptation .................................................................................... 38

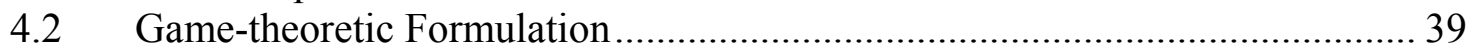

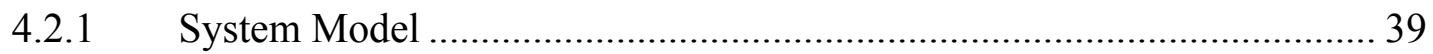

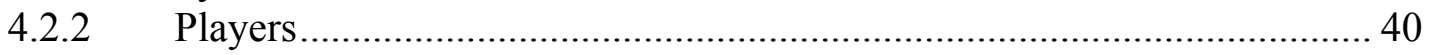

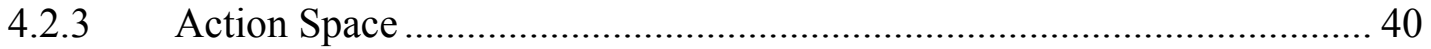

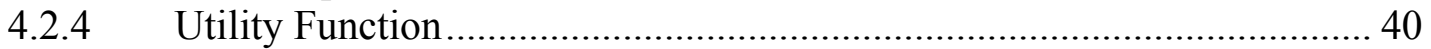

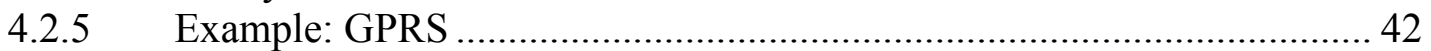

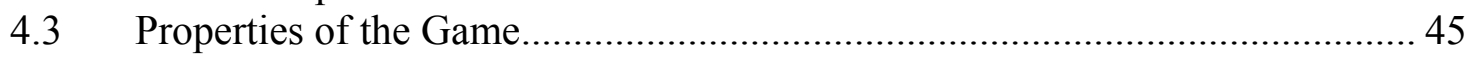

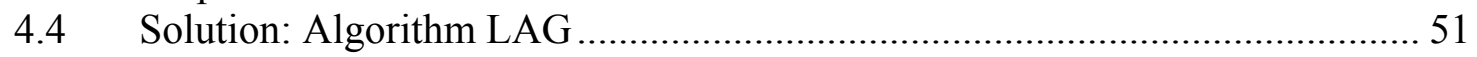

4.4.1 Convergence of Algorithm LAG .......................................................... 53

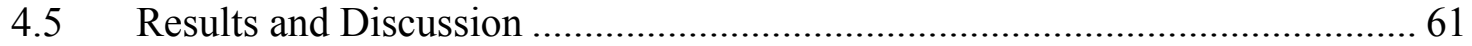

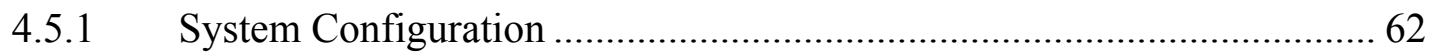

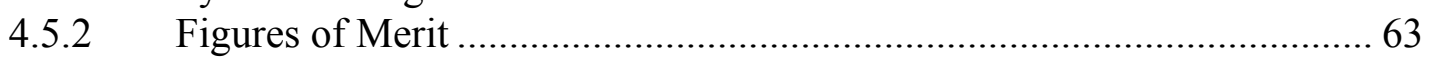

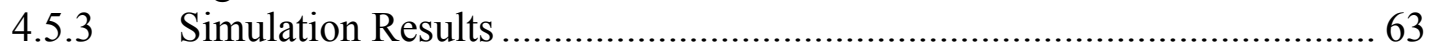

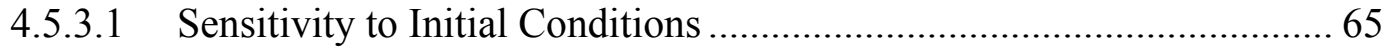

4.5.3.2 Impact of Penalty Function Parameters ................................................ 66

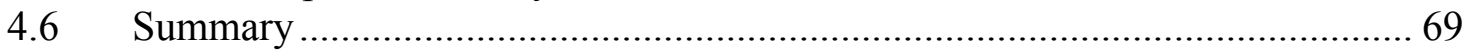


5 A Comparison of Game and System-theoretic Results........................................... 70

5.1 System Model and Problem Definition....................................................... 70

5.2 Optimum Target Assignment (OTA) …………….................................... 71

5.3 Stepwise Rate Removals Algorithm …………….......................................... 75

5.4 Simulation Results and Comparison ............................................................. 77

5.4.1 Interference-limited Scenario............................................................... 78

5.4.2 Noise-limited Scenario..................................................................... 79

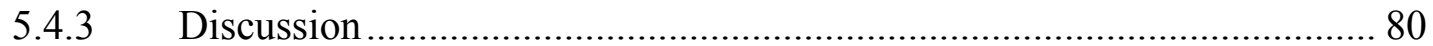

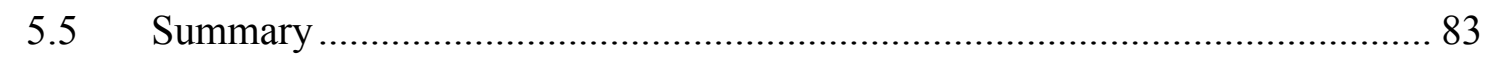

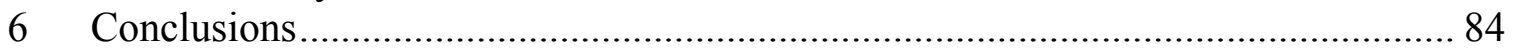

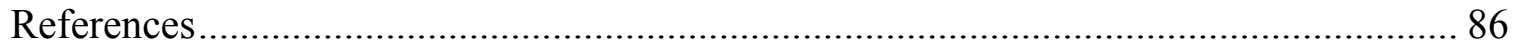




\section{List of Figures}

FIGURE 1-1 EVOLUTION OF GLOBAL CELLULAR STANDARDS.

FIGURE 1-2 EXAMPLE GPRS THROUGHPUT VERSUS SINR SIMULATION CURVES [73] SHOWING COMPARISON OF PERFORMANCE USING DIFFERENT CODING SCHEMES (CS1 - CS-4). THE Y-AXIS IS IN UNITS OF KILOBYTES PER SECOND (KBPS). (TU-50: TYPICAL URBAN FADING CHANNEL, MOBILE SPEED $=50 \mathrm{KM} / \mathrm{HR} . \mathrm{FH}=$ FREQUENCY HOPPING.).

FIGURE 2-1 SYSTEM MODEL OF A TYPICAL NON-CDMA CELLULAR SYSTEM, EMPLOYING SEVEN-CELL FREQUENCY RE-USE. THE SHADED CELLS ARE CO-CHANNEL INTERFERERS

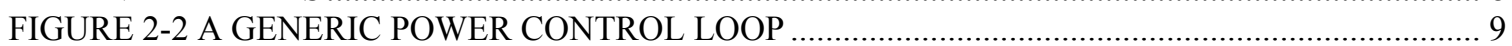

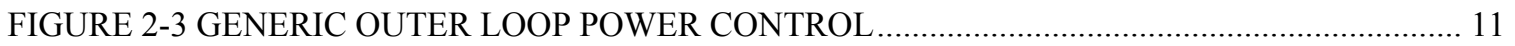

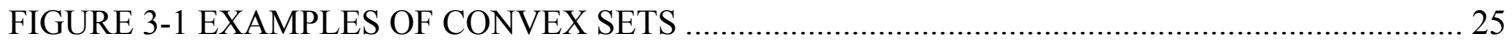

FIGURE 3-2 EXAMPLES OF NON-CONVEX SETS …….............................................................. 26

FIGURE 3-3 EXAMPLES OF CONCAVE, QUASI-CONCAVE AND NON-CONCAVE FUNCTIONS 27

FIGURE 3-4 SHAPE OF UTILITY FUNCTION IN [51] ......................................................................... 32

FIGURE 3-5 INFORMATION-THEORETIC CAPACITY $[F(X)]$ VERSUS SINR $[X]$ OF A BSC

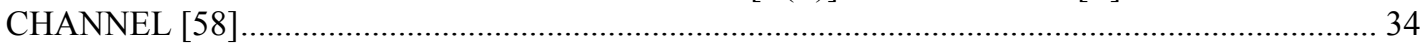

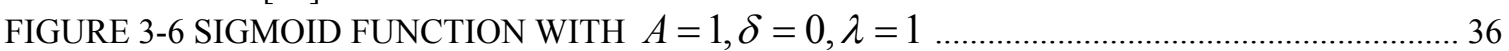

FIGURE 4-1 PENALTY FUNCTION AS A FUNCTION OF POWER FOR DIFFERENT INDICES $Q . .41$

FIGURE 4-2 EXAMPLE GPRS THROUGHPUT VERSUS SINR SIMULATION CURVES [73] SHOWING COMPARISON OF PERFORMANCE USING DIFFERENT CODE RATES. THE YAXIS IS IN UNITS OF KILOBYTES PER SECOND (KBPS). (TU-50: TYPICAL URBAN FADING CHANNEL, MOBILE SPEED $=50 \mathrm{KM} / \mathrm{HR}$. FH = FREQUENCY HOPPING.) …........... 43

FIGURE 4-3 RESULTS OF SIGMOIDAL CURVE-FITTING TO SIMULATION DATA FOR CS-3. THE DASHED CURVE IS THE APPROXIMATION

FIGURE 4-4 UTILITY FUNCTION IN A 2-PLAYER GAME, AS A FUNCTION OF POWER, FOR DIFFERENT RATES

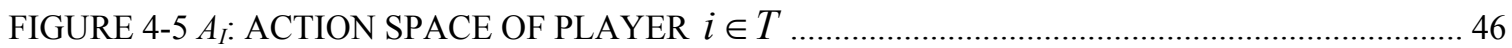

FIGURE 4-6 DEMONSTRATION OF QUASI-CONCAVITY OF THE UTILITY FUNCTION.............. 48

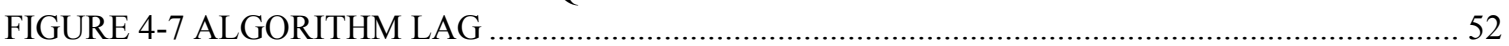

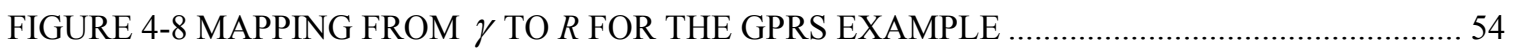

FIGURE 4-9 SEVEN-PLAYER GAME, RE-USE FACTOR = 3. A SET OF CO-CHANNEL CELLS IS

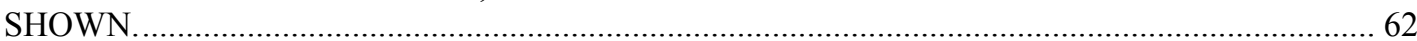

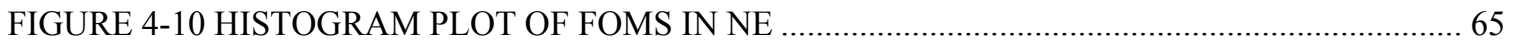

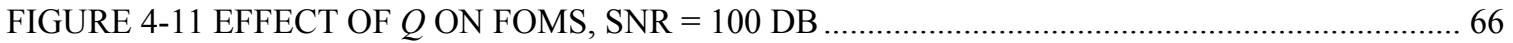

FIGURE 4-12 CLOSE-UP OF SYSTEM THROUGHPUT OF FIGURE 4-11 ……................................. 67

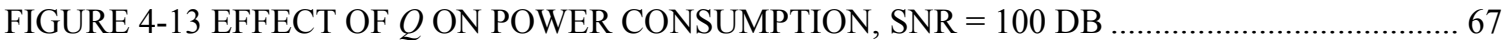

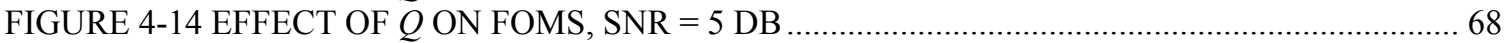

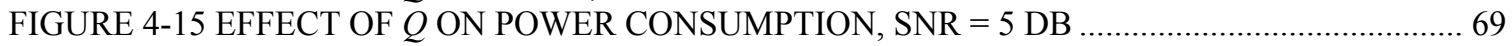

FIGURE 5-1 PERFORMANCE COMPARISON OF GSPC-GRR, GRP, SRR, OTA AND ALGORITHM LAG USING CDF OF SYSTEM THROUGHPUT (KBPS) FOR THE INTERFERENCE-LIMITED SCENARIO (SNR $=100$ DB). NOTE THAT THE PERFORMANCES OF SRR AND OTA ARE NEARLY IDENTICAL. 


\section{List of Tables}

TABLE 2-1 QUALITATIVE COMPARISON OF POWER CONTROL ALGORITHMS ....................... 14

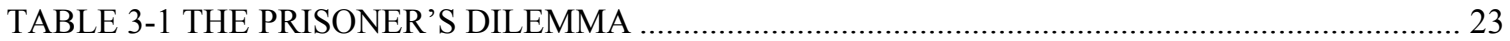

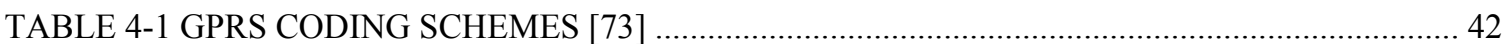

TABLE 4-2 PARAMETERS OF SIGMOID MODELS FOR THROUGHPUT VS. SINR CURVES ........ 42

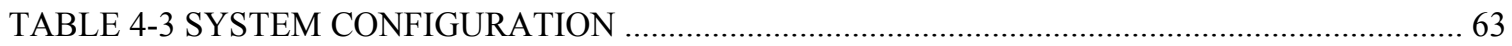

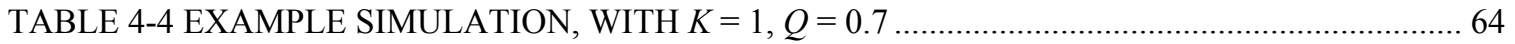

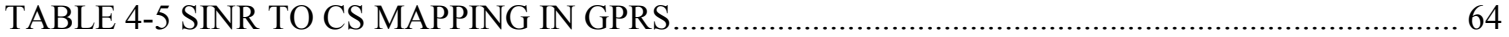

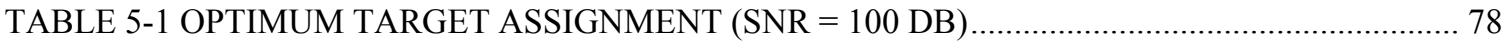

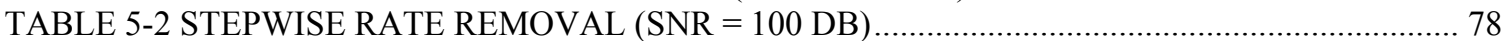

TABLE 5-3 GENERALIZED SELECTIVE POWER CONTROL (SNR = 100 DB) $\ldots \ldots \ldots \ldots \ldots \ldots \ldots \ldots \ldots \ldots \ldots \ldots . . . . . . . . . . . . . . . .78$

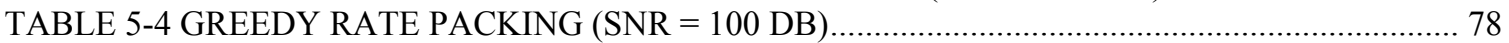

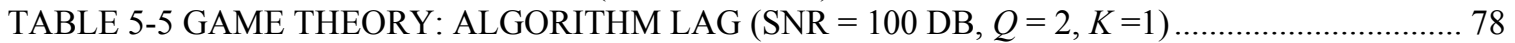

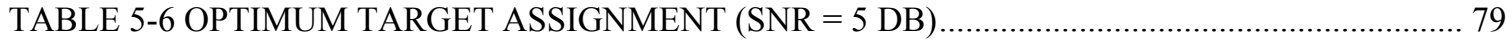

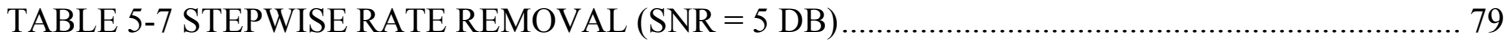

TABLE 5-8 GENERALIZED SELECTIVE POWER CONTROL (SNR = 5 DB) ……........................... 79

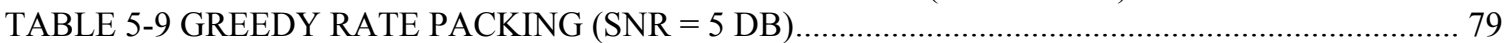

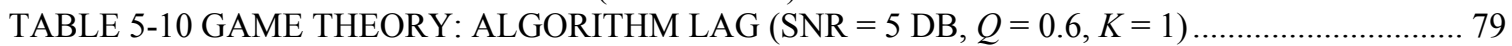

TABLE 5-11 COMPARISON OF RESULTS BASED ON FOM 1 AND FOM 2 VALUES ..................... 80

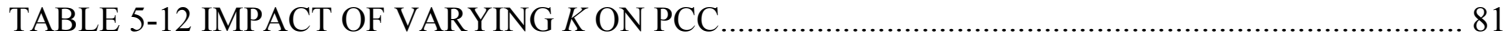

TABLE 5-13 GSPC WITH TARGET LIMITATION $(\mathrm{SNR}=100 \mathrm{DB}, \mathrm{MAX}$. TARGET $=30 \mathrm{DB}) \ldots \ldots \ldots . .82$

TABLE 5-14 PERCENTAGE SYSTEM CAPACITY IMPROVEMENT OBTAINED BY ALGORITHM

LAG 83 


\section{List of Acronyms}

\begin{tabular}{|l|l|}
\hline $2 \mathrm{G}$ & Second Generation \\
\hline 3G & Third Generation \\
\hline ALP & Adaptable Link Parameter \\
\hline AWGN & Additive White Gaussian Noise \\
\hline BER & Bit Error Rate \\
\hline BS & Base Station \\
\hline C/I & Carrier to Interference Ratio \\
\hline CDMA & Code Division Multiple Access \\
\hline CS & Coding Scheme \\
\hline DBA & Distributed Balancing Algorithm \\
\hline DCPC & Distributed Constrained Power Control \\
\hline EDGE & Enhanced Data Rates for Global Evolution \\
\hline FER & Frame Error Rate \\
\hline FDMA & Frequency Division Multiple Access \\
\hline FOM & Figure of Merit \\
\hline GMSK & Gaussian Minimum Shift Keying \\
\hline GPRS & General Packet Radio Service \\
\hline GRP & Greedy Rate Packing \\
\hline GRR & Gradual Rate Removals \\
\hline GSM & Global System for Mobile Communications \\
\hline GSPC & Generalized Selective Power Control \\
\hline IMT & International Mobile Telecommunications \\
\hline ITU & International Telecommunications Union \\
\hline LAG & Link Adaptation Game \\
\hline MS & Mobile Station \\
\hline NE & Nash Equilibrium \\
\hline OL & Outer Loop \\
\hline OLPC & Outer Loop Power Control \\
\hline OTA & Optimum Throughput Analysis \\
\hline PC & Power Control \\
\hline PCC & Power Consumption Coefficient \\
\hline PSK & Phase Shift Keying \\
\hline QoS & Quality of Service \\
\hline RRM & Radio Resource Management \\
\hline SINR & Signal to Interference and Noise Ratio \\
\hline SNR & Signal to Noise Ratio \\
\hline SRR & Successive Rate Removals \\
\hline TDMA & Time Division Multiple Access \\
\hline TD-SCDMA & Time-Division Synchronous CDMA \\
\hline UL & Uplink \\
\hline & \\
\hline
\end{tabular}




\begin{tabular}{|l|l|}
\hline UMTS & Universal Mobile Telecommunications System \\
\hline WCDMA & Wideband CDMA \\
\hline
\end{tabular}




\section{Introduction}

\subsection{Third Generation (3G) Wireless Communications ${ }^{1}$}

At the time of writing, cellular operators are continuing to roll out Third Generation (3G) services in several markets worldwide. Historically, the earliest analog systems, e.g. AMPS, are referred to as First Generation systems. These were superseded by a plethora of Second Generation (2G) standards, which employ digital communications technology. The proven "killer application" for mobile telephony has been and continues to be voice. However, starting with $2 \mathrm{G}$ systems, rudimentary data services, such as text messaging, have proved to be feasible. $3 \mathrm{G}$ wireless systems constitute a significant technological advancement over existing $2 \mathrm{G}$ systems. They differ from $2 \mathrm{G}$ systems in that they combine voice and data services, signifying a convergence of wireless technology with the Internet and computing. 3G is expected to be driven by applications as diverse as multimedia messaging, email, video telephony, music, Internet gaming, and other data services. 3G standards are, therefore, designed to support traffic classes with differing characteristics. For such an ambitious catalog of services to be realizable, several exciting new technologies that are unheard of in $2 \mathrm{G}$ standards are being deployed. These technologies include turbo coding, link adaptation, which is studied in this thesis, packet scheduling, multiuser detection and antenna diversity, to name a few.

As per the requirements set forth by the ITU's IMT-2000 recommendations [3], in order for a network to be classified as a $3 \mathrm{G}$ network, it must be able to transmit wireless data at

- $144 \mathrm{kbps}$ to mobile users,

- $384 \mathrm{kbps}$ to pedestrian users, and

- 2 Mbps to fixed locations.

In addition, $3 \mathrm{G}$ networks must be able to achieve the following.

- Provide equally effective symmetrical and asymmetrical data transfer capability

- Improve the voice quality over what $2 \mathrm{G}$ networks offer

- Increase the capacity for a given bandwidth

- Simultaneously support multiple classes of traffic with differing quality of service (QoS) requirements

- Permit global roaming for users

Requirements such as these have ensured that $3 \mathrm{G}$ technologies are far more sophisticated than $2 \mathrm{G}$ technologies. The performance analysis of $3 \mathrm{G}$ systems is likely to be complicated by a vast number of system variables, whose impact on system performance will be better understood with experience.

\footnotetext{
${ }^{1}$ The acronyms used in this chapter are defined in the List of Acronyms in the front matter.
} 


\subsection{G: Evolution and Status}

Figure 1-1 illustrates the evolutionary path followed by $2 \mathrm{G}$ standards to $3 \mathrm{G}$. The $2.5 \mathrm{G}$ standards are an intermediate step in this evolution.
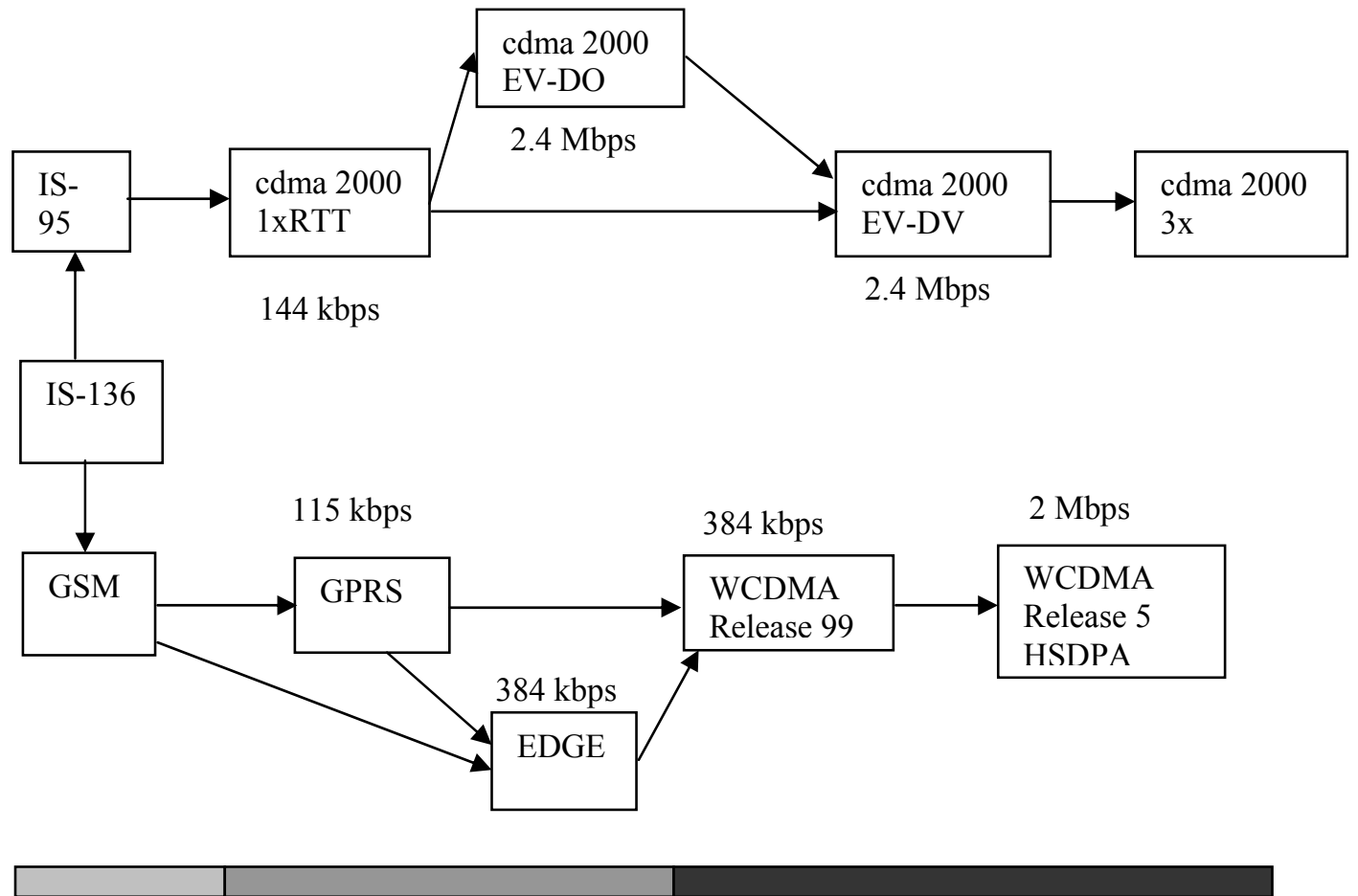

$2 \mathrm{G} \quad 2.5 \mathrm{G} \quad 3 \mathrm{G}$

Figure 1-1 Evolution of global cellular standards ${ }^{2}$

The enormous expenditure on spectrum licenses for $3 \mathrm{G}$ and the lukewarm response received from the consumer market have served to delay the deployment of $3 \mathrm{G}$ systems. Market research by Ovum, the largest European-headquartered advisor on telecoms, software and IT services, published in 2003, shows that, today, there are approximately 1.1 billion cellular subscribers worldwide [1]. Of these, less than 30 million subscribe to a $2.5 / 3 \mathrm{G}$ service. This statistic is an indicator that we are only in the nascent stage of $3 \mathrm{G}$ growth.

It is interesting to note that the ultimate $3 \mathrm{G}$ technologies will revolve around CDMA, of which cdma 2000 and WCDMA form two mutually incompatible standards (China's inhouse TD-SCDMA deserves mention as a domestic if not global contender). In general, the legacy $2 \mathrm{G}$ standard at the root of the $3 \mathrm{G}$ evolution path has dictated the direction adopted by migrating operators. $3 \mathrm{G}$ made its first appearance in the trendsetting Japanese market, where NTT's UMTS faced off with rival KDDI's cdma 2000 1x for the first

${ }^{2}$ Opinion on the classification of technology into the $2.5 \mathrm{G}$ or $3 \mathrm{G}$ categories is divided. In some quarters, cdma $20001 x$ RTT is regarded as being a 3G technology. It also remains unlikely that cdma $20003 x$ will ever be deployed. 
time. In GSM-dominated Europe, there has been a delay in the deployment of true $3 \mathrm{G}$ UMTS networks but GPRS and EDGE networks have proven to be a stopgap measure. For the carriers offering CDMA (Verizon and Sprint PCS) in the United States, upgrading to cdma $20001 \mathrm{x}$ has been an automatic choice. The TDMA carriers (AT\&T and Cingular Wireless) have decided to revamp their networks to GSM/GPRS/EDGE. The South Korean market is dominated by CDMA, hence an upgrade to cdma 2000 1x was the inevitable decision. Quoting an iGilliot report, dated April 2003 [2], "most of the $3 \mathrm{G}$ networks commercially operating are CDMA $1 \mathrm{x}-46$ networks worldwide and 21 more expected by the end of 2003. There are also 4 commercial CDMA 1x EV-DO networks operating and 3 more expected by the end of the year. While lagging behind on commercial network launches to date, by the end of 2003 there will be at least 12 UMTS commercial network launches". Interesting developments in the cellular industry can be expected in forthcoming years.

\subsection{Radio Resource Management (RRM) for 3G Networks}

Radio resource management [4] is the field of systems engineering which relates to the optimum utilization of the limited resources available in a wireless network. The performance of a wireless network depends on several parameters, including, but not limited to, bandwidth, load, frequency re-use, maximum power allocation, topography, and terminal speed. The impact of different technologies must often be factored into a performance study. The ultimate allocation of resources must be satisfactory for the user as well as the provider of the services. For example, CDMA networks present unique challenges not found in TDMA or FDMA. Often several resources must be traded off against each other, such as loading, measured by the number of users in the system or cell, versus link performance, measured by the bit error rate (BER). System performance can be evaluated using performance metrics such as system throughput, power consumption, and coverage probability. RRM involves the utilization of mathematical analysis and simulation to study the impact of the aforementioned parameters on system performance, as measured by the performance metrics. The results of such analysis and simulation translate into the implementation of favorable RRM schemes in network design. The areas of power control, handoff, admission control, and scheduling can be classified as belonging to the rather broad field of RRM.

In $2 \mathrm{G}$ networks, voice is the predominant application (circuit-switched data is the only other option). As a result, variable QoS need not be provisioned. Of greatest interest is the number of users that can be supported at an acceptable voice quality in a given bandwidth and coverage area [5]. Since voice is circuit-switched, for TDMA and FDMA based technologies, a fixed number of channels are available in a given bandwidth. The design parameters that affect average signal to interference and noise ratio ${ }^{3}$ (SINR), a measure of link quality, need to be optimized. In addition, the ability to provide an acceptable SINR to all the users in the network - in other words, the coverage probability

\footnotetext{
${ }^{3}$ Other common terms substitutable for SINR are signal to interference ratio (SIR) and carrier to interference ratio (C/I). In this thesis, we distinguish between SNR and SINR; the former incorporates only noise while the latter, both interference and noise.
} 
- must be evaluated. Of further interest are the call blocking and call dropping probabilities, which are functions of the number of available circuit-switched connections, i.e., the load [5]. In CDMA networks, there is the capability to increase capacity at the cost of degradation to voice quality. Achieving a delicate balance between these two requirements, where the first satisfies the provider of the service and the second the end-user, is an important network design challenge.

RRM of $3 \mathrm{G}$ networks is vastly different from that of $2 \mathrm{G}$ networks. There are several options available in $3 \mathrm{G}$ in terms of data rates and QoS. Some services, such as web browsing are delay tolerant, whereas others, such as voice and even more so, videoconferencing are delay intolerant. Thus, the homogeneity in service requirements for voice-only systems no longer exists for $3 \mathrm{G}$ systems. Moreover, system performance is now a function of a greater number of variables. Since $3 \mathrm{G}$ services will use a combination of packet-switched and circuit-switched channels, throughput and delay are more relevant metrics for common types of packet-switched data services, such as file transfer and web browsing. Technologies unique to $3 \mathrm{G}$ systems, such as scheduling and link adaptation, create new challenges in RRM. The techniques used in the analysis of voice networks need to be modified or supplemented by newer ones to address these challenges.

In this thesis, traditional analytical approaches to solving problems in RRM are categorized under "systems theory." In Chapter 2, we focus on the important RRM area of transmitter power control. A useful review of power control literature from a systemtheoretic perspective is provided. It includes a review of significant research on multi-rate power control. Systems techniques involve the application of linear algebra, queuing theory, and optimization to RRM problems. These techniques have found successful application in the analysis of $2 \mathrm{G}$ networks and are evolving to find application to the new needs of RRM of $3 \mathrm{G}$ networks.

Recently, new techniques utilizing game theory have come to light, especially in their application to the power control problem. Game theory is heretofore little understood or utilized by wireless systems engineers. It is the subject of extensive research in economics and the study of human interaction. Chapter 3 contains a self-contained introduction to game theory and the mathematical foundations of the game-theoretic ideas that are relevant to this work. The recent applications of game theory to the RRM of cellular systems that have shown it to be a promising and novel alternative to systems theory are also reviewed therein.

This thesis attempts to further contribute to existing work on RRM for $3 \mathrm{G}$ networks using game theory and systems theory as parallel, but contrasting approaches to the same end. We have selected a problem pertinent to a generic $3 \mathrm{G}$ network, that of multi-rate power control or link adaptation and power control. Our literature review in Chapter 3 reveals that this problem has not been studied sufficiently using game-theoretic models. While the approach adopted parallels earlier approaches to the power control problem, the problem we solve is a generalization of these other works. To support users with variable link quality, $2.5 \mathrm{G}$ and $3 \mathrm{G}$ systems permit switching between the members of a set of 
multiple code rates, modulation schemes, or spreading factors. The switching is conducted as a function of link quality and, hence, is appropriately called link adaptation. Considering, as an example, the next-generation standards evolving from GSM [6], we observe that in GPRS there is a choice of four coding schemes. In EDGE, there is the added choice of 8-PSK modulation over the existing GMSK of GSM/GPRS. In WCDMA [7], there exists the ability to adjust the spreading factor of a physical channel in response to varying link conditions. The different degrees of coding gain or, more generally, energy efficiency afforded by multiple rates and modulation schemes affect the average transmitter power consumption of the link, which suggests that power control and link adaptation can be carried out jointly. While decreasing the code rate improves energy efficiency, it might also result in lower throughput when channel conditions improve to the extent that the extra redundancy becomes unnecessary. However, further degradation may mean that the degree of redundancy provided by the code is insufficient to balance the losses of entire frames of information, which once again results in lower throughput. A typical set of link adaptation simulation profiles [73], shown for GPRS in Figure 1-2, illustrates the impact of varying code rate over various received SINR values, which are an indicator of link quality. The advantages of link adaptation over using a fixed code rate are evident from these curves.

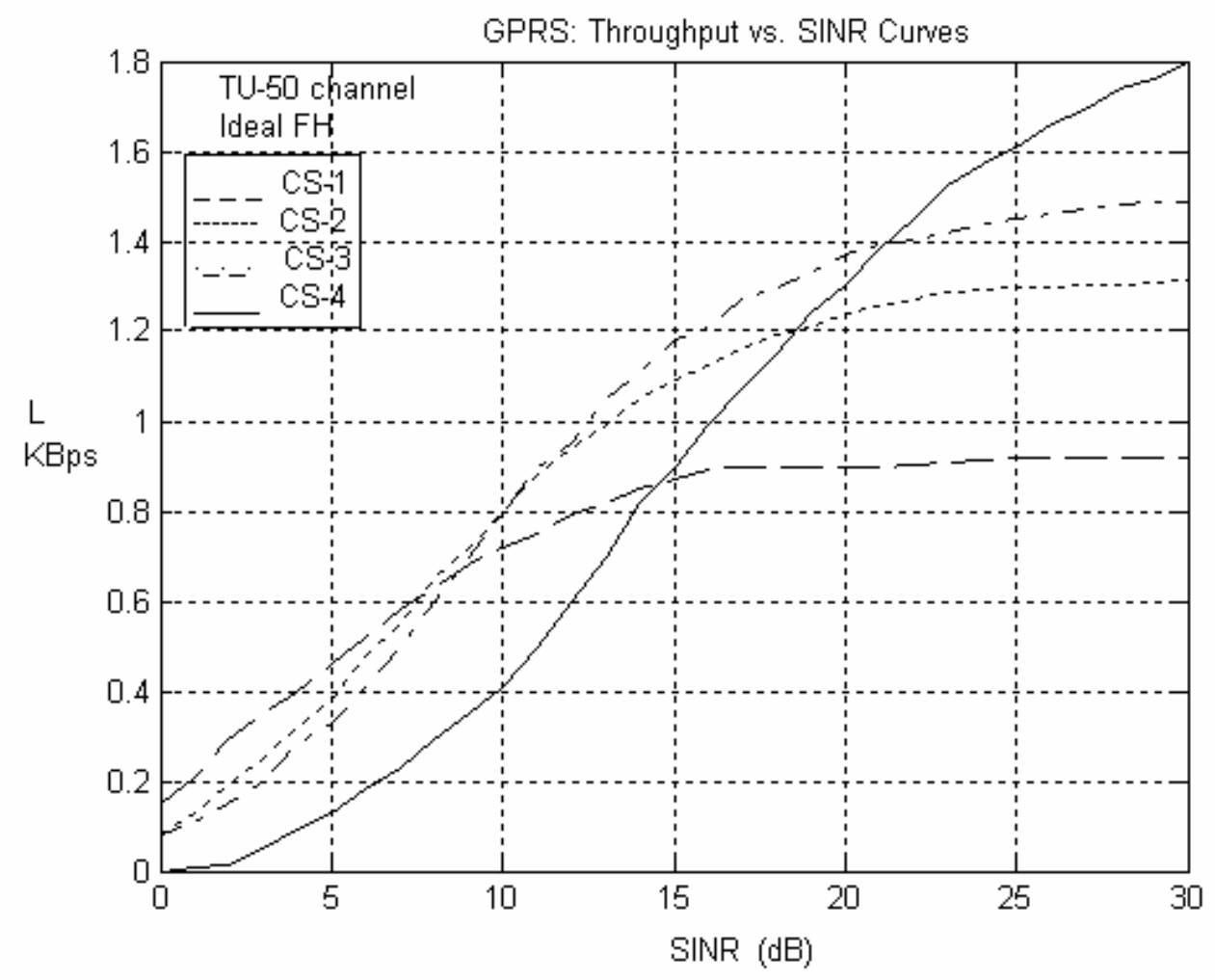

Figure 1-2 Example GPRS throughput versus SINR simulation curves [73] showing comparison of performance using different coding schemes (CS-1 - CS-4). The y-axis is in units of kilobytes per second (KBps). (TU-50: Typical Urban fading channel, mobile speed $=50 \mathrm{~km} / \mathrm{hr}$. FH $=$ Frequency Hopping.)

As a running example in this work, we study a GPRS network. The existence of a discrete set of code rates disturbs the otherwise well-behaved nature of this problem. 
Elementary results from game theory are applied to construct a model for the problem at hand and derive a solution in Chapter 4. In the next chapter, a novel solution to the same problem using systems theory is presented. This approach is not distributed and frames our problem as a system optimization. The two approaches are compared with each other and other system-theoretic solutions from the literature. We believe that that this often ignored exercise is important in validating game-theoretic analyses.

This thesis provides evidence that game theory is a viable alternative for solving RRM problems. Its usefulness will be evidenced by the emergence of new applications that are amenable to analysis using techniques from game theory. In Chapter 6, we conclude with some thoughts and observations on the utility of game theory within the scope and limitations of our research. 


\section{Power Control in RRM}

\subsection{Introduction to Power Control}

In Chapter 1, we introduced the subject of radio resource management (RRM), mentioned several important areas in RRM, and identified some of the RRM challenges presented by $3 \mathrm{G}$ networks. One ubiquitous and important area within RRM is that of transmitter power control or simply power control (PC). Power control has been studied for the last four decades. The earliest work on power control appears to be by Axelby and Osborne [8].

Transmitter power control is the adjustment of the transmitter power to achieve a desired link quality, e.g. receiver power level or signal to interference ratio. There are two factors that make power control necessary in cellular systems:

- The time-varying wireless propagation environment, which includes path loss, shadowing and small scale fading, and

- Co-channel interference

In cellular systems, several cells in a coverage area re-use frequencies and, hence, the transmissions in these co-channel cells interfere with each other. Co-channel interference is arguably the biggest impediment to increasing system capacity. In Figure 2-1, a sevencell frequency reuse scheme, in which the cells are represented by hexagons, is illustrated. A subset $C$ of one group of co-channel cells is shaded. A close-up of cell $i \in C$ is also shown in Figure 2-1. Let there be $N$ users in the system. Since each of the users is part of a wireless link, which in turn belongs to a cell, we use the terms users, cells and links interchangeably depending on the context. We focus on the downlink case in the following discussion. The link gain on a typical link is a positive real number less than one that includes both large-scale and small-scale fading. The link gain from transmitter or interferer (in this case the base station) $j$ to the receiver (in this case the mobile) in cell $i$, is denoted by $G_{i j}$. $G_{i i}$ is the link gain from the transmitter to receiver in cell $i \in C$. If the base-station (BS) transmitter power on the link in cell $i \in C$ is denoted by $\mathrm{P}_{i}$, and the thermal noise power at the receiving mobile station (MS) in cell $i \in C$ by $n_{i}$, the signal to interference and noise ratio (SINR) at MS $i, \gamma_{i}$ is given by the relation in Equation (2-1). A similar expression can be derived for the uplink.

$$
\gamma_{i}=\frac{G_{i i} P_{i}}{\sum_{j=1, j \neq i}^{N} G_{i j} P_{j}+n_{i}}
$$

It is convenient to use matrix notation to describe the link gains $G$. Let $\mathbf{G}=\left\{G_{i j}\right\}$ be the link gain matrix of the system described in Figure 2-1. Each element $(i, j)$ of the matrix simply represents the link gain from BS $i$ to MS $j$. The value of SINR that a link $i \in C$ strives to achieve is referred to as an SINR target and is denoted by $\gamma_{i}^{t}$. The matrix 
$\mathbf{H}$, defined in Equation (2-2) in terms of the targets and $\mathbf{G}$, is called the normalized link gain matrix.

$$
\begin{aligned}
H_{i j} & =\left\{\gamma_{i}^{t} G_{i j} / G_{i i}\right\}, \quad i \neq j \\
& =\{0\} \quad, i=j
\end{aligned}
$$

The link gain matrix and its normalized form will be used extensively throughout this thesis. The transmitter powers can be expressed in the form of a vector $\mathbf{P}=\left[P_{1}, P_{2}, \ldots, P_{N}\right]^{T}$, where the $P_{i}$ 's are defined as in Equation (2-3).

$$
P_{i} \in\{p \mid p \in \mathbb{R}, p>0\}, i \in C
$$

We similarly define the normalized noise vector $\boldsymbol{\eta}=\left[\eta_{1}, \eta_{2}, \ldots, \eta_{N}\right]^{T}$ and define its component $\eta_{i}$ in Equation (2-4). The normalized noise vector also appears later in this thesis.

$$
\eta_{i}=\left\{\frac{\gamma_{i}^{t} n_{i}}{G_{i i}}\right\}, n_{i}>0, \quad i \in C
$$

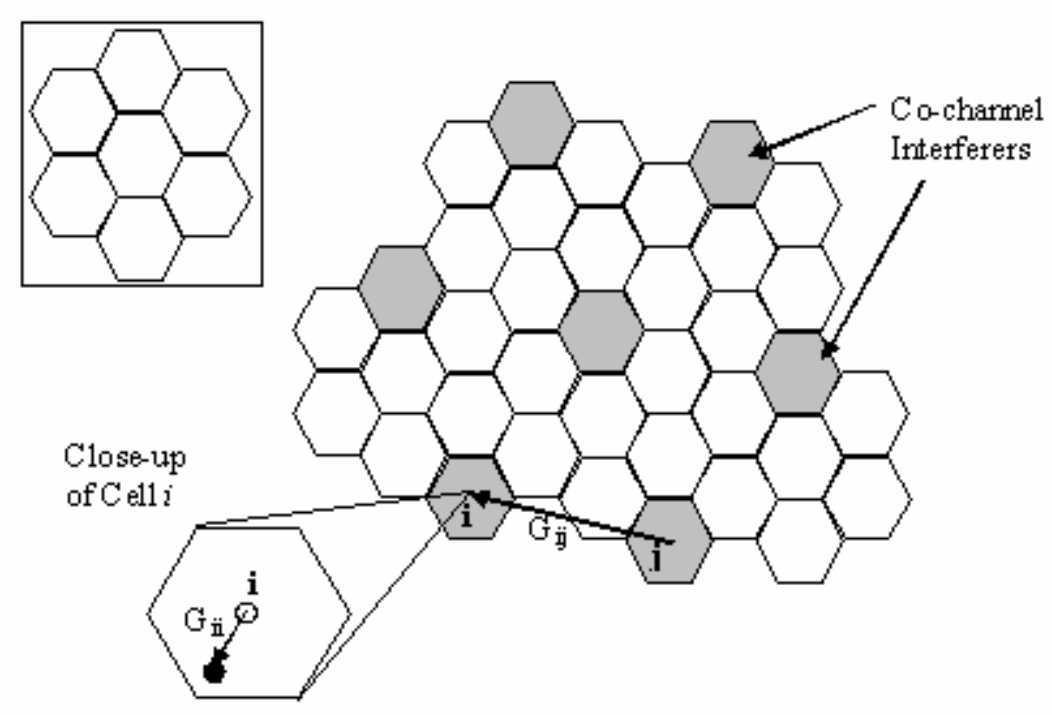

Figure 2-1 System model of a typical non-CDMA cellular system, employing seven-cell frequency re-use. The shaded cells are co-channel interferers

To achieve satisfactory quality on a wireless link, a certain SINR must be maintained. The quality of service (QoS) requirement for voice is uniform throughout the network, i.e. all voice links must achieve the same BER. The same may not be the case for data networks. In CDMA networks the requirements of power control differ for the downlink and uplink. However, an ideal power control algorithm will adjust transmitter power $P$ such that the exact SINR requirement, or some other link quality measure, is achieved, if at all possible. In our work, we consider receiver SINR to be the link quality measure. Increasing the power causes unnecessary interference to other co-channel links and 
wastes capacity. However, an inadequate amount of power will result in the deterioration of link quality.

The nature of the wireless propagation environment results in fluctuations of both the average signal strength, due to slow large-scale fading, as well as the instantaneous signal strength due to fast small-scale fading. The link path gain coefficients $G$ vary as a function of the MS position and movement in a coverage area. The power control algorithm must respond to these fluctuations in link quality by adjusting $P$ in a timely manner so that SINR remains at the target value that ensures satisfactory link quality.

\subsection{Power Control Terminology}

Based on this understanding of the requirements of power control, a block diagram of a power control loop is shown in Figure 2-2. The implementation of the functionality in the blocks depends on the details of the cellular system or standard. However, a channel quality estimate is compared with a desired target value of that estimate and the new power level is determined accordingly.

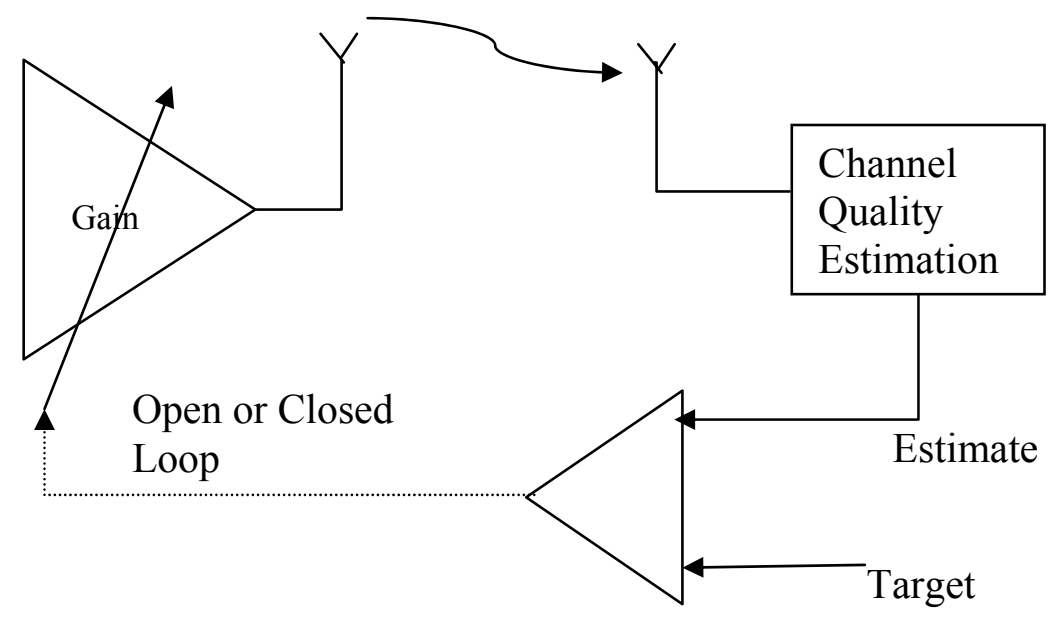

Figure 2-2 A generic power control loop

Depending on where the estimate is obtained, we can have open loop or closed loop power control. In an open loop power control scheme, a channel quality measurement made at the transmitter, assuming similar fading characteristics at the transmitter to those found at the receiver, is used to drive the transmitter power. However, while this reciprocity assumption is likely to be valid in forming an estimate of the path loss and shadowing, it is unlikely to be able to compensate for small scale fading [9]. The reason for this is that the frequency separation between the uplink and the downlink exceeds the coherence bandwidth [5] of the fading channel, rendering their small-scale fading components uncorrelated. The advantage of open loop power control is that it obviates 
feedback from the receiver and the delay associated with it. It also tends to mitigate fast fading by averaging it out. A closed loop power control scheme involves communication between the receiver, where the channel quality estimation occurs, and the transmitter, where the power level is appropriately adjusted. In slow fading this is necessary, since deep fades tend to be much longer than the duration of a symbol. However, the inherent feedback delay means that in a fast fading channel the channel quality may change before the new power level takes effect, rendering the closed loop ineffective.

Typically, for reasons mentioned in the preceding paragraph, the closed loop algorithm needs to operate at a much greater frequency than the open loop algorithm. Thus, a classification of power control based on the frequency of power control loop operation is possible. However, in general, fast power control is used to combat instantaneous fast fading effects, while slow power control is used to counteract large-scale propagation effects such as path loss and shadowing. A fast power control loop typically updates at intervals that are comparable to the coherence time [5] of a fading channel.

Channel quality estimation is essential for the successful operation of power control. Channel quality can be estimated from received signal strength, received SINR, or a moving average BER. In our work, the received SINR is the channel quality metric of choice. We further assume that SINR (we drop the "received" prefix henceforth) is estimated perfectly at the receiver.

\subsubsection{CDMA Power Control}

In CDMA, power control is slightly different on the uplink and downlink [10]. On the uplink of CDMA voice systems, there is a possibility that distant users' transmissions may be much weaker than those of near users in what is known as the near-far effect. It is shown in [11] that the performance of a multiple-access spread spectrum link using nonorthogonal codes is optimized when all users are received with equal power. The main purpose of power control on the UL of CDMA voice systems is to alleviate the near-far effect.

On the CDMA downlink, users are separated by orthogonal Walsh (or similar) codes. However, power control is necessary to reduce interference caused to other cells which share the same code set. With universal frequency reuse normally being implemented, neighboring cells might interfere with each other. Multipath propagation delays also result in the codes of different users no longer being time-synchronous at the MS. This makes it necessary to control downlink power and the result is an increase in network capacity.

Two terms, inner loop and outer loop power control (OLPC), have been popularized in CDMA parlance. The inner loop was described earlier in Figure 2-2. The outer loop [12] is a target adjustment algorithm in which the cyclic redundancy check (CRC) carried out on each frame determines whether that frame was in error or not. The target referred to here is the set-point of the closed inner loop. If in error, the target is increased by a particular up-step; if not, it is decreased by a down-step. These steps are typically 
measured in decibels $(\mathrm{dB})$. If an $x \%$ FER is desired, the relation between the up-step and the down-step is given by Equation (2-5).

$$
U p=(100-x) \text { Down }
$$

For example, an FER of 1\% would mean that, on average a frame error can be expected to occur once in every 100 frames. Hence, there should be 100-1 = 99 correct frames, corresponding to down-steps, for each erroneous frame.

In general, an outer-loop can be described as being any algorithm that finds the target utilized by the inner loop. A logical representation of an OL algorithm appears in Figure $2-3$.

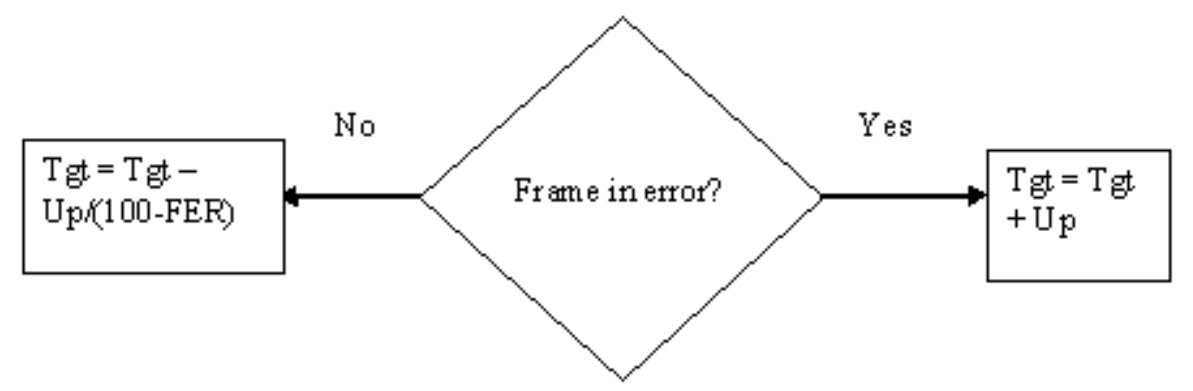

Figure 2-3 Generic outer loop power control

In a cellular system that supports packet-switched connections, as was mentioned in Chapter 1, it is often feasible to support users at different throughputs. This usually depends on the rate assigned to a user. Different rates often have different optimum targets. This thesis is related to OLPC in that it focuses on algorithms and analytical techniques that determine the selection of the rates in packet-switched data networks that optimize network performance based on some performance criteria. It is assumed that fast fading can be counteracted by a fast power control inner loop.

\subsection{Power Control Theory}

Power control is recognized as a vital RRM technique to counteract fading and cochannel interference and in the process, improve system capacity. Early work in this area understandably focused on voice systems, to be later extended and adapted to data systems. We now provide a brief overview of significant contributions to the literature on the theory of power control. Power control algorithms can be categorized as being centralized or distributed. In centralized schemes, signaling within the BS system is required to exchange system-wide power allocation information. This permits a single entity in the BS system to execute power control. In distributed schemes, each link consisting of a BS and MS can independently compute the appropriate amount of power required without externally supplied knowledge of the same process being executed in other links. Centralized schemes are impractical to implement but theoretically useful in that they can provide bounds on the achievable SINR in every member belonging to a set 
of co-channel links. Distributed schemes form the basis of practical power control algorithms. An overview of work in this area can be found in Chapter 4 of [13].

A notable early effort [14] proposed keeping received power at a fixed level. This scheme was subsequently outperformed by other schemes. One of these is described in [15], in which the author approaches the problem of the optimum achievable SINR in a purely interference-limited system.

\section{Definition 2-1 Achievable Signal to Interference and Noise Ratio}

A particular SINR $\gamma^{t}$ is said to be achievable if every link $i \in C$ can achieve an SINR equal to or in excess of it. Mathematically, this condition translates to Equation (2-6).

$$
\gamma_{i}>\gamma^{t}, \forall i \in C
$$

In [15], the author proposes a "C/I balancing" scheme in which he shows that the maximum achievable downlink SINR in the network is given by Equation (2-7).

$$
\gamma_{\max }=\frac{1}{\lambda_{\max }}
$$

Here $\lambda_{\max }$ is the largest real eigenvalue [16] of the matrix $\mathbf{Z}$ defined in Equation (2-8).

$$
Z_{i j}=\frac{G_{i j}}{G_{i i}}
$$

The links are said to be balanced when the condition in Equation (2-9) is satisfied.

$$
\gamma_{i}=\gamma_{j}, \quad \forall i, j \in C
$$

The same paper [15] asserts that C/I balancing can be extended to the uplink. Then, the author proposes an iterative Stepwise Removal Algorithm (SRA) in which cells that do not exceed the SINR target are made to stop transmitting. The iterations continue until all such cells have been silenced and $\gamma_{\max }$ for the remaining cells exceed the target SINR. In other words, the condition of Equation (2-10) must be satisfied, where $C^{\prime}$ is the set obtained by iteratively applying SRA starting with $C$.

$$
\gamma_{i}=\gamma_{j}=\gamma_{\max }, \quad \forall i, j \in C^{\prime}
$$

A distributed version of this algorithm, called the Distributed Balancing Algorithm (DBA) was suggested in [17]. The algorithm starts with an arbitrary initial approximation to $\mathbf{P}$, and uses the update Equation (2-11).

$$
P_{i}^{n+1}=\beta P_{i}^{n}\left[1+\frac{1}{\gamma_{i}^{n}}\right], \beta>0
$$

Here $n$ is the iteration count and as $n \rightarrow \infty$, it is shown that $\gamma_{i} \rightarrow \gamma_{\max }, \forall i \in C$. This algorithm has been shown to converge by an application of the power method for finding the dominant eigenvalue of a matrix [16]. Convergence proves to be slow in the case of a high target $\mathrm{C} / \mathrm{I}$. A practical limitation of this method is that an appropriate value of $\beta$ 
cannot be computed in a distributed manner. A Limited Information SRA algorithm based on the DBA is also proposed in [17].

The schemes mentioned here consider cellular systems to be strictly interference-limited, but in [18], a distributed algorithm that considers noise in the system is proposed. This work suggests that user $i$ change its SINR in proportion to its offset from a predefined SINR target. Mathematically, this is equivalent to the update iteration of Equation (2-12). As before, $n$ is the iteration count.

$$
\gamma_{i}^{n+1}-\gamma_{i}^{n}=-\beta\left(\gamma_{i}^{n}-\gamma^{t}\right)
$$

Here, $\beta>0$ is an arbitrary positive constant. Assuming that the interference remains constant during the power update interval, the update equation can be shown to be equivalent to Equation (2-13).

$$
P_{i}^{n+1}=P_{i}^{n} \frac{\gamma^{t}}{\gamma_{i}^{n}}
$$

Written in matrix form using Equation (2-1), Equation (2-2), Equation (2-3) and Equation (2-4) in Equation (2-13), we arrive at Equation (2-14) below.

$$
\mathbf{P}^{n+1}=\mathbf{H} \mathbf{P}^{n}+\boldsymbol{\eta}
$$

Provided the target $\gamma^{t}$ is achievable as per Definition 2-1, the algorithm is shown to converge to the following solution, obtained by setting $\mathbf{P}^{n+1}=\mathbf{P}^{n}$ in Equation (2-14), since convergence occurs when the powers are the same between two consecutive iterations.

$$
\mathbf{P}=(\mathbf{I}-\mathbf{H})^{-1} \boldsymbol{\eta}
$$

Here, I is the identity matrix. The proof of convergence follows from the Jacobi relaxation method (see [4] for a description and the proof). The power-update scheme proposed at the time was synchronous between users, but subsequent research in [19] has shown that the same result can be achieved asynchronously.

In all the work summarized until now, power is assumed to be an infinite resource. Hence, the next logical step was the development of algorithms for constrained power control. Perhaps the most renowned of these is the Distributed Constrained Power Control (DCPC) algorithm [20]. The necessary modification to the update Equation $(2-13)$ is shown in Equation (2-16).

$$
P_{i}^{n+1}=\min \left\{P_{\max }, P_{i}^{n} \frac{\gamma^{t}}{\gamma_{i}^{n}}\right\}
$$

Here, $P_{\max }$ is the peak power available to that link. Grandhi, et al. [20] prove that DCPC converges to a fixed point provided that $\gamma^{t}$ is achievable (Definition 2-1).

We now summarize the goals of power control algorithms. A list of desirable properties of power control algorithms should include the following.

- $\quad$ They should be distributed 
- They should be optimum with respect to some criterion

- They should converge rapidly in a wide variety of cases

- They should avoid the "party effect" in which convergence does not take place and power on each co-channel link continuously increases up to its maximum

- They must support use of constrained power

- Ideally, they should be amenable to implementation in an actual network

In summary, the algorithms discussed in this section, when evaluated in accordance with these criteria, compare as shown in Table 2-1.

Table 2-1 Qualitative Comparison of Power Control Algorithms

\begin{tabular}{|l|l|l|l|}
\hline Property & C/I Balancing & DBA & DCPC \\
\hline Distributed & No & Not strictly & Yes \\
\hline Avoids Party Effect? & Yes & No & No \\
\hline Constrained Power & No & No & Yes \\
\hline Implementation & No & Impractical & Yes \\
\hline Rate adaptive & No & No & No \\
\hline
\end{tabular}

\subsection{Multi-rate Power Control}

An interesting observation in Table 2-1 (indicated by the italicized row) is that none of the algorithms discussed so far are able to jointly carry out link adaptation and power control. The requirement that a single system-wide SINR target be attained is responsible for this limitation. Link adaptation or adaptive modulation refers to the ability to tradeoff energy efficiency for spectral efficiency as a function of link quality. It might be carried out by selecting one of a number of different modulation, coding, or spreading schemes, or some combination of these schemes. For example, GPRS [6] offers four coding schemes with Gaussian Minimum Shift Keyed (GMSK) modulation. In EDGE [6], the more spectrally-efficient 8-PSK modulation may be used in addition to multiple code rates to further boost data rates, while in WCDMA [7], several spreading codes and coding schemes are available to support an even wider variety of rates. It is, therefore, evident that in next-generation networks, adaptive modulation will be one of the means of achieving the most efficient use of spectrum. The theory of power control has been extended to involve power control of users with a number of adaptable rates, namely multi-rate power control. The SINR target and rate are directly related. Higher rates are supported at higher SINR values and vice-versa. Hence, the assignment of SINR is synonymous with the assignment of rates. We now survey some of the significant literature in this area. A more comprehensive summary appears in Berggren's dissertation [21].

Qiu and Chawla [22] address the maximization of total throughput, given a set of users and the corresponding path gain matrix $\mathbf{G}$. The total throughput is defined as the sum of 
individual user throughputs. The expression for user $i$ 's throughput, in terms of its SINR $\gamma_{i}$ and the power vector $\mathbf{P}$, is given by Equation (2-17).

$$
T\left(\gamma_{i}\right)=\log _{2}\left(1+k \gamma_{i}(\mathbf{P})\right)
$$

Here, $k$ depends on the modulation or coding scheme being used. To solve this constrained non-linear optimization problem, where constraints are on power, the authors suggest iterative non-distributed algorithms, for each of two objective functions - the first, the sum of throughputs, and the second the product of individual SINRs. Both solutions employ the method of feasible directions [23]. The first algorithm suffers from a tendency to converge to sub-optimal solutions depending on the initial guess for $\mathbf{P}$, even though it can potentially lead to optimal throughput, whereas the second is shown to converge to a unique global, albeit slightly sub-optimal, maximum. However, since this work does not address the minimization of power resources, both algorithms result in at least one user transmitting at maximum power.

In [24], Wu addresses inner loop power control in the context of CDMA systems, in which the assumption of a single system-wide SINR target is relaxed. Instead, each link can be assumed to have heterogeneous thresholds. It is assumed that the outer loop sets a feasible SINR target for the inner loop to achieve. This work neglects the effect of background noise, assuming the system to be strictly interference-limited. The proposals in [24] are essentially extensions of the algorithms suggested for C/I balancing, in either a centralized or quasi-distributed manner.

An early paper to address combined power control and link adaptation is [25]. In this work Kim, et al. relax the assumptions that the rates must be continuous. They then propose two power control algorithms. The first is an iterative algorithm that maximizes instantaneous system throughput using the method of Lagrangian multipliers [26]. This algorithm first computes target rates using the Lagrangian technique, which are then used to solve for power using DCPC [20]. In the second, called Selective Power Control (SPC), they consider the problem of throughput maximization together with minimization of energy consumption. The two algorithms are shown to perform favorably compared to results in [22].

A more recent effort [27] frames the resource allocation problem, again in the context of CDMA networks, as the following optimization problem: determine the allocation of powers $\mathbf{P}$, rates $\mathbf{r}$, and base station assignments in single cell and multi-cell scenarios that maximize the objective function $Z$ defined in Equation (2-18).

$$
Z=\sum_{i=1}^{N} \lambda_{i} r_{i}-\lambda_{h} h^{l}
$$

The terms in Equation (2-18) are defined below.

$\lambda_{i}=\gamma_{i} f\left(\tau_{i}\right)$

$\gamma_{i}=$ Target SINR for user $i$

$f\left(\tau_{i}\right)=A+B \exp \left(\frac{-\tau_{i}}{D}\right)$, where A,B,D are positive constants 
$\tau_{i}=$ Residual delay

$r_{i}=$ Rate

$\lambda_{h}=$ Cost associated with handoff

$h^{l}=$ Number of handoffs associated with BS assignment $l$

The optimization is subject to typical constraints on power and rate. Moreover, a solution is attempted for single-cells as well as multiple cells. However, the work does not explicitly focus on lowering energy consumption, since it does not include power as an optimization criterion.

In Berggren's dissertation [21], the minimization of power consumption in a CDMA system is explicitly specified as an optimization criterion. The optimization problem presented therein is as described in Equation (2-19).

$$
\begin{gathered}
\min _{P_{j}} \sum_{j=1}^{N} P_{j} \\
\text { s.t. } \sum_{j=1}^{N} R_{j}=\tilde{R}
\end{gathered}
$$

(where $\tilde{\mathrm{R}}$ is the desired throughput for the cell)

$$
0 \leq P_{j} \leq P_{\max }
$$

In Equation (2-19), $R_{j}$ is the throughput of user $j$. The minimum power solution of Equation (2-15) is then derived for the uplink of a CDMA system, as shown in Equation (2-20).

$$
P_{i}^{*}=\frac{I+n_{i}}{1-\sum_{j=1}^{N} \frac{\gamma_{j}}{1+\gamma_{j}}} \cdot \frac{\gamma_{i}}{G_{i}\left(1+\gamma_{i}\right)}
$$

In Equation (2-20) $G_{i}$ is the link gain from $\mathrm{MS} i$ to its $\mathrm{BS}$, and $I$ is the inter-cell interference. The aforementioned optimization problem and its constraints are suitably modified using Equation (2-20). This leads to a heuristic solution algorithm for the same, called Greedy Rate Packing (GRP) [21]. We show how GRP may be adapted to nonCDMA systems in Section 2.4.1. In GRP, the maximum feasible rate is allocated to each mobile, starting with the one having the best channel (hence the name "greedy"). Furthermore, GRP is shown to minimize total power by assigning SINR in proportion to the prevailing channel conditions. In other words, if $G_{i}>G_{j}$ then $\gamma_{i}>\gamma_{j}$. In [21], GRP is also extended to the CDMA downlink.

The same work [21] also generalizes the SPC algorithm of [25] and calls it Generalized Selective Power Control (GSPC). GSPC is shown to be optimal, given feasibility, i.e. every mobile can be supported at its maximum rate within the feasible range of powers. In the case of infeasibility, a technique of gradual rate removals (GRR) is suggested. In GRR, the rate of the user with the minimum channel gain, amongst those that do not meet their SINR targets, is reduced. This procedure is repeated until the system becomes feasible. GSPC and GRR are described in greater detail in Section 2.4.1. 
Yet more related literature exists, for example, [28], [29] and [30]. In [28], a distributed algorithm that discovers the throughput-maximizing $\mathbf{P}$ and $\mathbf{r}$ vectors is proposed, and the network is shown to have a Nash Equilibrium. The concept of Nash Equilibrium [33] will be studied further in Chapter 3. Furthermore, multiple Nash Equilibria are identified. In [29], the power control and rate allocation problem that maximizes throughput while meeting a specific FER target is shown to be NP-complete. A heuristic algorithm that achieves the stated objective is then proposed. In [30], the minimization of the sum of powers and maximization of the sum of rates are separately considered as objectives, and the corresponding non-linear optimization problems are formulated. Suggestions for their solution are also offered.

\subsubsection{GRP and GSPC}

An important aspect of our work is the comparison of our results with those obtained using some of the techniques described earlier. We have decided to use the following.

- Greedy Rate Packing (GRP)

- Generalized Selective Power Control with Gradual Rate Removals (GSPC-GRR)

We now describe these algorithms in greater detail and adapt them to the system model in Figure 2-1. In GRP, users are assigned rates (actually SINR, which is synonymous with rate) in an order determined by their relative signal strengths. The algorithm is greedy since it assigns SINR to the users with best link quality first, and assigns the remaining capacity to the weaker users. Equation $(2-20)$ is not valid for non-CDMA systems. Hence, it is necessary to modify GRP to suit our purposes. We do not distinguish between the uplink and downlink. Let us assume that the users are sorted such that $G_{11}>G_{22}>\ldots>G_{N N}$. Each user $i \in C$ is assigned an SINR such that $\gamma_{\min }<\gamma_{i} \leq \gamma_{\max }$. Initially $\mathbf{P}=0$. We set an iteration counter $k=1$ and count up to $N$. At each iteration $k$, the following steps are executed.

1. Assuming the worst-case interference, assign the maximum SINR within the available power $P_{k, \max }$, to user $k$. This is equivalent to selecting $\gamma_{k}$ according to Equation (2-21).

$$
\gamma_{k}=\max \left(\gamma_{\min }, \min \left(\frac{P_{k, \text { max }} G_{k k}}{\sum_{i=1}^{k-1} G_{k i} P_{i, \text { max }}+n_{k}}, \gamma_{\max }\right)\right)
$$

The reason for assuming worst-case interference is that the preceding users $i<k$ must be favored by the greedy nature of the scheme, i.e. their targets must not be compromised for succeeding users. The worst case for these users is that they attain their current targets at peak power consumption $P_{i, \max }$. This in turn leads to the upperlimit on interference received at $k$. Thus, $\gamma_{k}$ must be selected in a manner that is 
consistent with this requirement. Following this line of reasoning, we obtain Equation (2-21).

2. Calculate $P_{k}$ as follows.

$$
P_{k}=\min \left(\gamma_{k} \frac{I_{k}}{G_{k k}}, P_{k, \max }\right)
$$

Here, $I_{k}$ is the actual interference at $k$.

$$
I_{k}=\sum_{j=1, j \neq i}^{N} G_{k j} P_{j}+n_{k}
$$

Note that the manner of choosing $\gamma_{k}$ in Equation (2-21) implies that $P_{k} \leq P_{k, \max }$. Also, note that, for any user $j>k$, the power $P_{j}$ required to achieve the same $\gamma_{k}$ will be greater than $P_{k}$, since by our ordering of users, $G_{j j}<G_{k k}$.

3. Using Equation (2-22) and Equation (2-23), recalculate $P_{i}$ in descending order of $i$, where $i \in\{k-1, k-2, \ldots, 1\}$. This order is important since any user $j$ calculates its required power based on the updated powers of all lower priority users $i>j$. Due to the addition of user $k, P_{i}$ will have increased for all users.

4. Recalculate $\gamma_{i}$ for $i \in\{1,2, \ldots, k\}$ using Equation (2-1). This reflects the latest power updates. Note that $\gamma_{1}$ will be unchanged, for the highest priority user. However, for all other $i, \gamma_{i}$ will have decreased from its value at the last iteration. The decrease follows by noting that in Step 3, the recalculation of power for user $j$ does not take into account the increased interference caused by the increasing powers of users $i<j$, i.e. the higher priority users. Hence, the degradation of $\gamma_{i}$ will be most severe for the users experiencing poor channel conditions.

Note that, at any iteration $k$, the power increase $\Delta P_{i}(k)$ experienced by user $i$, which follows from step 3, is shown in Equation (2-24). The summation is over the set of all lower priority users.

$$
\Delta P_{i}(k)=\sum_{j=i+1}^{k}\left(\frac{\gamma_{i}(k-1) G_{i j}}{G_{i i}}\right) \cdot \Delta P_{j}(k)=\sum_{j=i+1}^{k} H_{i j} \Delta P_{j}(k)
$$

From Equation (2-24) it is apparent that by assigning rates (SINR) in the prescribed order, i.e. $G_{11}>G_{22}>\ldots>G_{N N}$, the power increases are more likely to be minimized for a given target assignment, since $H_{i j}$ is more likely to be reduced in the case of a higher $G_{i i}$. However, unlike in the case of the CDMA uplink, the power is not guaranteed to be minimized. This is contingent on the ratios $G_{i j} / G_{i i}$ and not $G_{i i}$ alone. However, using $G_{i i}$ for the ordering is simple and more practical.

GSPC works in a different manner. In this algorithm, it is assumed that each user has a number of different available rates in bits per second. We assume that a user $i \in C$ has 
$K_{i}$ different rates, chosen from a set of rates $R=\left\{r^{1}, r^{2}, \ldots, r^{\left(\max _{i \in C} K_{i}\right)}\right\}$ such that its set of rates $R_{i}$ can be written in ascending order as $R_{i}=\left\{r^{1}, r^{2}, \ldots, r^{K_{i}}\right\}$. Let the rate assigned for any user $i \in C$ from $R_{i}$ be denoted by $r_{i}$. We define the shorthand for a rate increment as follows: if $r_{i}=r^{m}$, then $r_{i}+1=r^{m+1}$. Similarly, a rate decrement involves a simple sign change. The target that link $i$ is required to achieve is a function of $r_{i}$ and is denoted by $\gamma_{i}^{t}\left(r_{i}\right)$. Corresponding to the set of rates $R_{i}$, we define a set of targets $\Gamma_{i}^{t}=\left\{\gamma_{i}^{t}\left(r^{m}\right) \mid r^{m} \in R_{i}, m=1,2, \ldots, K_{i}\right\}$. Hence, $\gamma_{i}^{t}\left(r_{i}\right) \in \Gamma_{i}^{t}$.

Initially, each user is assigned its lowest rate, i.e. $r_{i}=r^{1} \forall i \in C$. The GSPC algorithm then proceeds as follows. Until the powers of all the users have converged, the algorithm described in the following two steps, A and B, is iterated. Step B is known as GRR. It is required if any users have been assigned a rate that they might not be able to achieve after the assignment of every user has been accomplished by GSPC (step A). GRR works by penalizing the weakest users by decrementing their maximum rates.

Thus, at any iteration $k$, if $\exists i$ such that $\left|P_{i}(k+1)-P_{i}(k)\right|>\varepsilon$, where $\varepsilon>0$ is an arbitrarily small tolerated error, perform the following steps:

A. For all $i \in C$

1. $r_{i}(k)=r^{1} \forall i \in C$

2. While $r_{i}(k)<r^{K_{i}}$

a. If the rate $r_{i}(k)$ is achievable within the maximum power, or, mathematically,

$$
\frac{\gamma_{i}^{t}\left(r_{i}(k)\right) P_{i}(k)}{\gamma_{i}(k)}<P_{i, \max }
$$

increment to the next higher rate, as shown in Equation (2-26). Here, $\gamma_{i}$ is defined in Equation (2-1).

$$
r_{i}(k)=r_{i}(k)+1
$$

b. Else go to step 3 .

3. Compute the power required to achieve the highest achievable $r_{i}(k)$ resulting from step 2.

$$
P_{i}(k+1)=\frac{\gamma_{i}^{t}\left(r_{i}(k)\right) P_{i}(k)}{\gamma_{i}(k)}
$$

B. For all $i \in C$

1. If $\gamma_{i}(k+1)<\gamma_{i}^{t}\left(r_{i}(k)\right)$

a. Find the weakest user $j$. 


$$
j=\arg \min _{i \in C}\left(G_{i i}\right)
$$

b. Reduce maximum rate of $j$ by a single rate decrement.

$$
K_{j}(k+1)=K_{j}(k)-1
$$

c. Return to step A.

Thus, the two part procedure described in steps A and B is repeated until the powers of all users remain constant between any two iterations of the algorithm. The performances of GRP and GSPC are compared with our own contributions in Chapter 5.

\subsection{Summary}

This chapter presented a self-contained introduction to power control in cellular networks. A basic system model of a non-CDMA cellular system was presented in Section 2.1, and the importance of power control in RRM was clearly outlined. Additionally, key system concepts such as the link gain matrix and SINR were introduced for the first time in this thesis. These concepts will be repeatedly encountered throughout this document. In Section 2.2, important power control terminology was clearly spelt out, and an introduction to CDMA power control was provided. The underlying mathematics from linear algebra, which facilitates the system-level analysis of power control problems, was presented in Section 2.3. Several important examples from early power control literature were cited with intent to assist the reader in following the development of power control theory. The evolution of analytical techniques, from the early methods used for voice-only systems, to the more recent ones used to study data systems, was traced. The subject of multi-rate power control, which is the area of interest in this thesis, was given due attention in Section 2.4, accompanied once again by several examples. Detailed algorithmic implementations of two techniques, GRP and GSPC-GRR, were explained in the same section. Their performances form a basis for comparison with the new results presented later in this thesis. The following chapter introduces a new view of power control - one that uses game theory. 


\section{Game Theory and its Applications}

\subsection{Introduction to Game Theory}

To the unfamiliar reader, the term "game theory" might sound rather frivolous. In truth it is a vast and growing body of knowledge that is as applicable to markets as it is to describing animal behavior. Expressed succinctly, game theory is a set of tools designed to analyze the interactions between interdependent decision-making entities. Quoting from [31], "A game is a description of strategic interaction that includes the constraints on the actions that the players can take and the players' interests, but does not specify the actions that the players do take. A solution is a systematic description of the outcomes that may emerge in a family of games. Game theory suggests reasonable solutions for classes of games and examines their outcomes."

A more rudimentary description of game theory, offered by [32], portrays it as the scientific study of conflict of interest. Indeed, it is possible to imagine several such situations in which more than one individual's actions may determine their outcomes, and in which different individuals have differing preferences amongst the outcomes. Game theory enhances the understanding of conflict by devising theories, mathematical models and abstractions that serve to explain the nature and results of conflict. Several applications are listed later in this chapter.

There are a few basic assumptions underpinning game theory that facilitate the construction of tractable models for real situations whose complete details would perhaps be impossible to capture. The existence of such assumptions casts a shadow of doubt on the efficacy of game theory, but debating their limitations is beyond the scope of this thesis. A complete discussion can be found in [32], but here we will only state some of these assumptions.

First, it is assumed that each individual involved in a game, in other words, each player, has a definite ordering of preferences over all outcomes of a given situation. These preferences, when expressed numerically, take the form of a utility function. Each player wishes to maximize his (or her) utility function.

Second, it is assumed that the variables upon which the outcomes of a situation depend and the values that they can assume can be precisely characterized. Further, the decisionmakers in the game can be assumed to have complete knowledge of the actions and even the preferences of their peers.

These characteristics are often described by the term "rational." Thus, one way in which we can informally formulate a game is as a situation where there exist $n$ decision-making entities or players, whose choices or actions influence the outcomes of that situation. A player attaches a utility to each one of these outcomes and is motivated to act in a manner 
that maximizes his utility, given the choices of his peers. This characterization of an $n$ player game is known as the normal or strategic form of a game. In this form, each player makes his choices at the outset of the game without knowing the choices of the other players. There is no scope for a player to revise his strategy after making his choice. A contrasting model of a game is the extensive form. In the extensive form, it is possible to specify an order of play in which a player, at any stage of the game is able to assess the impact of previous actions before formulating his present strategy.

\subsection{Applications of Game Theory}

We now enumerate some applications of game theory to appreciate its usefulness in the analysis and solution of a diverse collection of problems. Although developed by mathematicians, game theory finds ready application to problems in the social sciences, especially economics, politics and military strategy. Several applications, both traditional and modern, are discussed in [33]. Some of these applications are elaborated upon in the following.

- Auctions - In an auction, a group of bidders place an ascending sequence of bids on a commodity until one bidder acquires some or the entire commodity. The ultimate going price of an item on auction arrives when all but the winning bidder are no longer willing to bid further. Each bidder is faced with the dilemma of selecting a price which ensures that he does not end up empty-handed and also does not simultaneously expend all his resources. Commodities that are traded at auctions include art, securities, and even wireless spectrum!

- Elections - In a typical election, several parties with selfish interests vote in an attempt to elect their preferred candidate into a position of authority, or vote for or against a statute. The preferences of any one party are likely to influence the decision making of one or more other parties. This makes an election a very interesting game.

- Markets - A company in a competitive market is confronted by several challenging questions - how much to produce, how much to invest in the creation of a new product, how to price the product, and so on. Often the answer to these questions depends on factors that are not entirely in control of the company. Inevitably, a company pays close attention to the business activities of its competitors in its endeavor to stay at the forefront of competition. At the same time, it must not ignore the expectations of its clients and shareholders. The analysis of economic markets is a traditional topic for game-theoretic analysis.

- Animal behavior - Recently, the competitive behavior among animals has been subjected to game-theoretic analysis. Competition arises on account of the scarce resources available to the animal population, including but not restricted to feeding, shelter and mating. 
- Random drug testing of sportspersons - To catch drug users in competitive sport, the international and domestic sporting governing bodies conduct random drug tests. Cheating athletes stand to improve their chances of victory at the cost of being banned from their sport, while the drug-testing agencies must deter athletes from taking drugs by conducting tests to trap cheaters on one hand and balance their budgets on the other.

\subsection{The Prisoners Dilemma}

In addition to the real-world examples mentioned above, several unrealistic examples can be constructed for pedagogical purposes. These help to capture the essence and nature of games and game theory. Perhaps, none is more famous than the Prisoners' Dilemma, which was invented by A.W. Tucker. In this simple game, two criminals A and B have been arrested in connection with a crime. The authorities possess sufficient evidence to convict both criminals with one-year sentences. However, both criminals are offered an escape clause. Each is told that if he confesses to the crime and the other does not, he will go free while the other is assigned a fifteen-year sentence. However, if both do end up confessing, they shall both be imprisoned for five years. This simple game is summarized in Table 3-1.

Table 3-1 The Prisoner's Dilemma

\begin{tabular}{c|cc} 
& 'A' Confess & 'A' Not Confess \\
\hline 'B' Confess & 5,5 & 15,0 \\
'B' Not Confess & 0,15 & 1,1
\end{tabular}

The players in this game are A and B. The strategies or actions available to them are \{Confess, Not Confess\}. The payoffs or utilities are the durations of their prison sentences, displayed as pairs in the cells of Table 3-1. Every utility pair consists of A's utility followed by that of B. Note that a lower utility is preferable.

The analysis of this game proceeds as follows. Both players know that by not confessing they can come away with light sentences. However, for either of them there is the attraction of getting away with no sentence by confessing, provided the other does not do likewise. Subsequently, both players end up confessing, and receive sentences of five years each. The final outcome of the game is known as the Nash Equilibrium. It is the most ubiquitous solution concept in game theory. A subsequent section of this chapter will formally present this key concept in game theory.

\subsection{A Brief History of Modern Game Theory}

We now trace the development of modern game theory through the years. The seminal work of Von Neumann and Morgenstern, encompassed in their masterwork Theory of Games and Economic Behavior [34] (1944), is regarded as the genesis of modern game theory. Their work essentially contained an axiomatic treatment of utility, detailed 
solutions of zero-sum games, and introduced cooperative game theory. John Nash then made perhaps the most influential contribution to game theory by introducing the idea of a Nash Equilibrium [35] (1950). In a Nash Equilibrium, no action in any player's action set can yield a superior utility to the current choice. As explained later, since this applies to all players, no player finds any means to improve his/her utility by unilaterally deviating from his/her equilibrium action. Further important contributions in the late 1960's and early 1970's were made by Reinhard Selten who applied the Nash Equilibrium concept to what are known as dynamic games [37]. Dynamic games involve time as a parameter, which implies that the timing of a player's action is as important as the actions itself. Of equal importance was John Harsanyi's extension of Nash's work to the domain of games with incomplete information [36]. In games with incomplete information, a player may have only partial or no information about the game situation.

Thus far, much attention has been devoted to non-cooperative game theory. There also exists a less popular field called cooperative game theory. The differences between the two can be encapsulated in the following [31]. When individual players make strategic decisions autonomously, we can classify the situation under non-cooperative game theory. When groups of players jointly make a strategic decision, we consider the situation to be a problem in cooperative game theory. Thus, cooperative game theory admits to the possibility of coalition formation amongst groups of players.

\subsection{Mathematical Concepts}

A few fundamental ideas from game theory are employed in this thesis. They can only be precisely described in mathematical terms. For completeness, we first review some preliminary results, which we shall refer to on several occasions in this thesis. Most of these results are basic to set theory and real analysis. All sets are assumed to belong to an $n$-dimensional Euclidean space, denoted by $E_{n}$.

\section{Definition 3-1 Bounded Set [23]}

A set $S$ in $E_{n}$ is said to be bounded if it can be contained within a ball of finite radius.

\section{Definition 3-2 Closed Set [23]}

A set $S$ in $E_{n}$ is said to be closed if it contains all points that are arbitrarily close to it. The set of all such arbitrarily close points is called the closure of $\mathrm{S}$ and is denoted by $\mathrm{cl} S$. A point $\mathbf{x} \in \operatorname{cl} S$ if, for each $\varepsilon>0, S \bigcap N_{\varepsilon}(\mathbf{x}) \neq \varnothing$, where $N_{\varepsilon}(\mathbf{x})=\{\mathbf{y}:\|\mathbf{y}-\mathbf{x}\| \leq \varepsilon\}$. Thus, a set is said to be closed if $S=\operatorname{cl} S$. 


\section{Definition 3-3 Compact Set [23]}

A compact set $S$ in $E_{n}$ is both closed and bounded. For every sequence in a compact set $S$, there must be a convergent subsequence with a limit in $S$.

A discrete-valued finite set is always closed and bounded. Hence, it is compact. A segment of the real line that includes its endpoints is also closed and bounded. Hence, it too is compact. The set of natural numbers is not bounded, and, hence, it is not compact. For the same reason, the real line is not compact either.

\section{Definition 3-4 Convex Set [23]}

A set $S$ in $E_{n}$ is convex if a line segment joining any two points in the set, lies entirely within that set.

Applying Definition 3-4, we conclude that the sets in Figure 3-1 are convex, whereas the sets in Figure 3-2 are not.

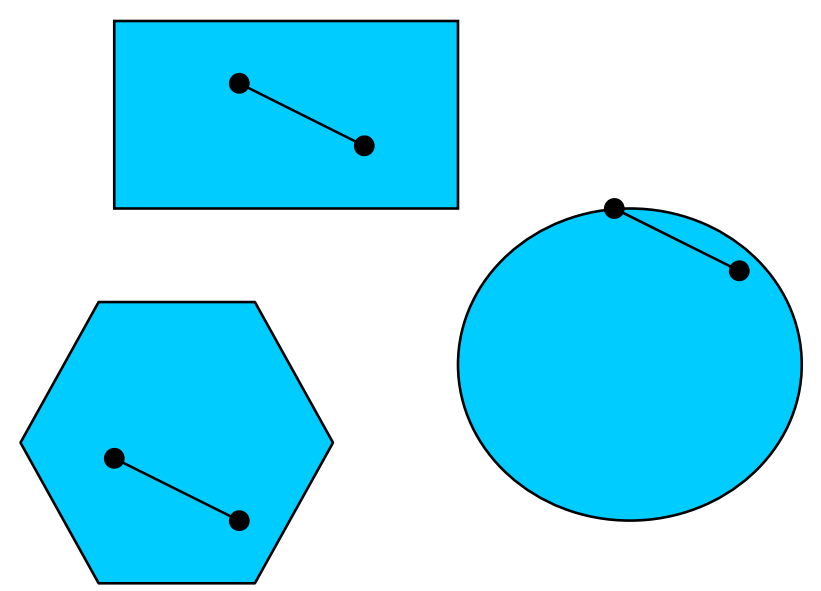

Figure 3-1 Examples of convex sets 


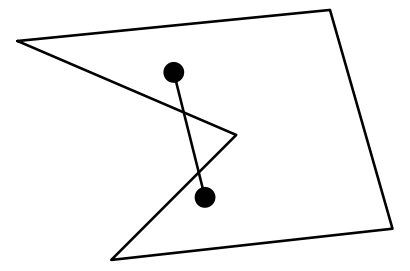

Figure 3-2 Examples of non-convex sets

\section{Definition 3-5 Concave Function [23]}

A function $f: S \rightarrow E_{1}$, where $S$ is a convex set in $E_{n}$, is said to be concave on $S$ if

$$
f\left(\lambda \mathbf{x}_{1}+(1-\lambda) \mathbf{x}_{2}\right) \geq \lambda f\left(\mathbf{x}_{1}\right)+(1-\lambda) f\left(\mathbf{x}_{2}\right)
$$

for each $\mathbf{x}_{1}, \mathbf{x}_{2} \in S$ and $\lambda \in(0,1)$. If the inequality in Equation (3-1) holds as a strict inequality, then $f$ is said to be strictly concave.

\section{Definition 3-6 Quasi-concave Function [23]}

A function $f: S \rightarrow E_{1}$, where $S$ is a convex set in $E_{n}$, is said to be quasi-concave on $S$ if

$$
f\left(\lambda \mathbf{x}_{1}+(1-\lambda) \mathbf{x}_{2}\right) \geq \min \left\{f\left(\mathbf{x}_{1}\right), f\left(\mathbf{x}_{2}\right)\right\}
$$

for each $\mathbf{x}_{1}, \mathbf{x}_{2} \in S$ and $\lambda \in(0,1)$.

If $-f$ is concave (quasi-concave), then $f$ is said to be convex (quasi-convex). In Figure 3-3, three functions are depicted. Here (a) is a concave function, (b) is convex, while (c) is quasi-concave.

\section{Definition 3-7 Strongly Quasi-concave Function [23]}

A function $f: S \rightarrow E_{1}$, where $S$ is a convex set in $E_{n}$, is said to be strongly quasi-concave on $S$ if

$$
f\left(\lambda \mathbf{x}_{1}+(1-\lambda) \mathbf{x}_{2}\right)>\min \left\{f\left(\mathbf{x}_{1}\right), f\left(\mathbf{x}_{2}\right)\right\}
$$

for each $\mathbf{x}_{1}, \mathbf{x}_{2} \in S, \mathbf{x}_{1} \neq \mathbf{x}_{2}$ and $\lambda \in(0,1)$. 


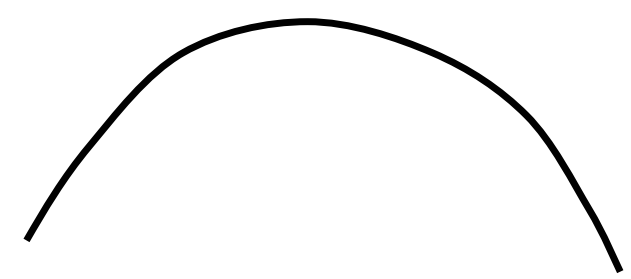

(a)

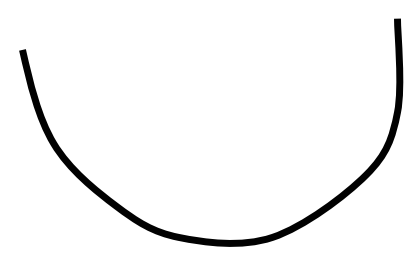

(b)

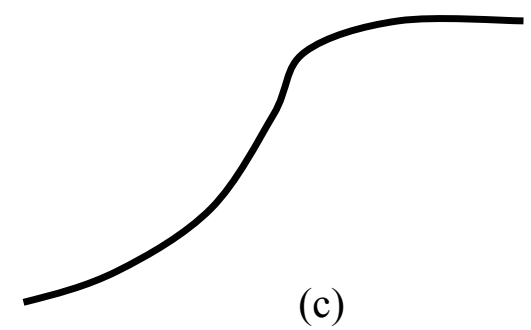

Figure 3-3 Examples of concave, quasi-concave and non-concave functions

\section{Definition 3-8 Continuous Function [23]}

A real-valued function $f: S \rightarrow E_{1}$, where $S$ is a convex set in $E_{n}$, is said to be continuous at $\overline{\mathbf{x}} \in S$ if, for any given $\varepsilon>0$, there exists $\delta>0$ such that, $\mathbf{x} \in S$ and $\|\mathbf{x}-\overline{\mathbf{x}}\|<\delta$ imply that $|f(x)-f(\overline{\mathbf{x}})|<\varepsilon$.

In this thesis, we apply the concept of a game in the strategic form. The definitions that follow pertain to strategic form games and will be utilized throughout the thesis.

\section{Definition 3-9 Strategic Form Game [31]}

A game $G$ in the strategic form consists of the following.

- A finite set of players, denoted by $N$.

- For each player $i \in N$, a non-empty set of actions, denoted by $A_{i}$. The action space of the game is given by $A=\times_{j \in N} A_{j}$, the Cartesian cross product of the action sets of individual players.

- For each player $i \in N$, a preference relation $R_{i}(a, b)$ is defined on $A$. The relation $R_{i}(a, b)$ is read as the action-tuple $a$ is preferable to another action-tuple $b$, for 
player $i \in N$. An action-tuple ${ }^{4}$, denoted by the shorthand, $\left(a_{i}\right)_{i \in N}$ can be considered to be the vector (or profile) of actions (or strategies) chosen by the players, one action per player. The notation $a_{-i}$ is used to indicate the profile of actions taken by all players except player $i$. Thus, it is possible to express an action tuple using the shorthand $a=\left(a_{i}, a_{-i}\right)$. The preference relation is usually represented in terms of a utility function $U_{i}: A \rightarrow \mathbb{R}$, where the relation $R_{i}(a, b)$ implies that $U_{i}(a)>U_{i}(b)$.

Recall that in the discussion of the Prisoner's Dilemma (Section 3.3), the solution was called the Nash Equilibrium (NE) of the game. This solution idea is the most ubiquitous in all game theory. From an intuitive standpoint, it is not possible for any player in equilibrium to profit by unilaterally deviating from his or her equilibrium action. However, it might be possible for all players to select new strategies such that a new NE is attained wherein all players benefit. In fact, the earlier NE is then said to be Pareto inefficient. It is possible for a game to possess more than one NE; hence, a NE need not be unique.

\section{Definition 3-10 Nash Equilibrium [31]}

The NE of a strategic game $G=\left\langle N, A, R>\right.$ is an action-tuple $a^{\prime}$ which satisfies the following property for all $i \in N$

$$
U_{i}\left(a_{i}^{\prime}, a_{-i}^{\prime}\right) \geq U_{i}\left(a_{i}, a_{-i}^{\prime}\right) \quad \forall a_{i} \in A_{i}
$$

\section{Definition 3-11 Nash Equilibrium (2) [31]}

An alternative but equivalent definition of a NE is possible in terms of the best-response function $B$. The best-response function of a player $i \in N$, given the set of actions $a_{-i}^{\prime}$ chosen by the other players, is the set of actions

$$
B_{i}\left(a_{-i}^{\prime}\right)=\left\{\hat{a}_{i} \in A_{i}: U_{i}\left(\hat{a}_{i}, a_{-i}^{\prime}\right) \geq U_{i}\left(a_{i}, a_{-i}^{\prime}\right), \forall a_{i} \in A_{i}\right\}
$$

A NE is the action-tuple $a_{i}^{\prime}$ for which $a_{i}^{\prime} \in B\left(a_{-i}^{\prime}\right), \forall i \in N$.

A NE represents the situation where each player is playing its best-response action. This latter definition of the NE points to a method of solving a game. If it is possible to compute the best-response function of each player, the simultaneous solution of the set of best response functions will be the NE. Note that a NE does not exist for every game. Before undertaking an attempt at solving the game, it is important to verify the existence of a NE using the Nash Existence Theorem [35] [38].

\footnotetext{
${ }^{4}$ In the analysis in Chapter 4, we use boldface vector notation to denote action-tuples. This is consistent with power control literature (see Chapter 2)
} 


\section{Theorem 3-1 Nash Existence Theorem [35]}

A strategic game $G=\langle N, A, R>$ has at least one NE if, $\forall i \in N$, the following conditions hold:

- the set $A_{i}$ of actions is a non-empty, convex polyhedral set in a Euclidean space;

- the preference relation is continuous on $A$ and quasi-concave on $A_{i}$.

This theorem is based on Kakutani's fixed point theorem [38]. The terms from set theory used in Theorem 3-1 are concisely defined in Definition 3-1 to Definition 3-8. There is an equivalent version of the Nash Existence Theorem attributed to Debreu [39], Glicksberg [41] and Fan [40]. This form of the theorem, which is stated in Theorem 3-2, directly employs the utility function and is, therefore, more convenient to use. To differentiate it from Theorem 3-1, we call it the Glicksberg-Fan Theorem.

\section{Theorem 3-2 Glicksberg-Fan Theorem [39][40][41]}

A strategic game $G=\langle N, A, U>$ has at least one NE if, $\forall i \in N$, the following conditions hold:

- the set $A_{i}$ of actions is a non-empty, compact and convex subset of a Euclidean space;

- the utility function $U_{i}$ is continuous on $A$ and quasi-concave on $A_{i}$.

An important point to note here is that the failure to comply with the requirements stipulated in Theorem 3-1 or Theorem 3-2 does not preclude the existence of a NE in a game. Thus, these conditions are sufficient, but not necessary.

Finally, we mention an important property for characterizing NE, that of Pareto efficiency or superiority. Informally speaking, a NE is said to be Pareto-optimal if it is not possible to choose another action-tuple that improves the utility of at least one player, while simultaneously not reducing the utilities of the others. In mathematical terms, the concept of Pareto efficiency is defined in Definition 3-12.

\section{Definition 3-12 Pareto Superiority and Optimality [53]}

An action-tuple $a^{\prime}$ is Pareto superior to another action-tuple $a$, if,

- $U_{i}\left(a^{\prime}\right) \geq U_{i}(a)$ for all $i \in N$

- $U_{j}\left(a^{\prime}\right)>U_{j}(a)$ for some $j \in N$

An action-tuple $\bar{a}$ is said to be Pareto optimal if there exists no other tuple that is Pareto superior to it. 


\subsection{Application of Game Theory to Power Control}

\subsubsection{Motivation}

Recently game theory has no longer been restricted to strictly economic problems. A growing number of applications in fields related to electrical engineering and computer science have appeared in recent literature. Examples include robotic control [43], scheduling problems [45], optimal routing [44], flow control [46], network admission [47], contention-based multiple access [48] and power control. A good starting point is the survey paper by MacKenzie and Wicker [42].

As we have pointed out earlier in this chapter, the rationality assumptions of game theory have been subjected to attack in economic circles. Due to the greater degree of determinism in the manner in which computing machines operate, these assumptions appear more plausible when applied to engineering situations. Specifically, machines possess limited intelligence relative to human beings, permitting the engineer to isolate a few significant variables by which he may wish to characterize the problem at hand. It may also be reasonable to assume that each decision-making entity possesses sufficient intelligence to be completely aware of the characteristics of peer decision-making entities. Very often, the decision makers in an engineering problem are approximately similar to each other, if not identical. This limited variability in their characteristics can be accommodated without making the model of the game excessively complicated. In a similar manner, it might often be possible to frame realistic and mathematically tractable utility functions that may be based on theoretical or empirical findings. Thus, there is likely to be less difficulty and controversy in applying the rich set of techniques existing in game theory to engineering problems.

In the context of this thesis, power control is the most interesting engineering-specific application area in game theory. It is fairly simple to imagine why game theory is a worthwhile tool in the system-level analysis of radio resource management (RRM) problems. The nodes in a wireless network compete for the use of scarce radio resources. Obvious examples are power, access to a shared channel in multiple-access scenarios, the allocation of spreading codes from a shared pool in adaptive multi-rate systems, and so on. The wireless environment is inherently interference-limited, which results in interaction between its nodes. By adopting the view that the nodes in a wireless network wish to selfishly appropriate as much of the shared radio resources as possible, it is possible to model situations that fit this description as non-cooperative games. In this thesis, we have applied these ideas to the link adaptation problem. The link adaptation problem can be viewed as an extension of traditional power control problems (see Chapter 2) to incorporate multiple rates. Therefore, adaptations of the set of techniques applied to problems in power control for voice-only systems can be used to solve problems in link adaptation for data systems. 


\subsubsection{Review of Literature}

Game theory was first proposed as a viable alternative for solving the power control problem for wireless data networks in a series of publications by Goodman, Mandayam, et al. [49] [50] [51] [53]. There are several similarities in the approach adopted by these authors to our own, which is described in Chapter 4. In this respect, their contribution serves as a template for application of strategic form game-theoretic techniques to power control problems. The breadth of their research is captured in [51].

In [51], the authors propose the model $G=\left[N,\left\{P_{j}\right\},\left\{u_{j}().\right\}\right]$ for their non-cooperative power control game (NPG). In the model $G, N=\{1,2, \ldots, \mathrm{N}\}$ is the set of players, $P_{j}$ is the strategy set of powers of player $j$, and $u_{j}$ is the utility function of player $j$. Each player selects a power level $p_{j} \in P_{j}$. The utility function of player $j$ is defined as

$$
u_{j}\left(p_{j}, p_{-j}\right)=\frac{L R}{M p_{j}} f\left(\gamma_{j}\right) \quad \frac{\text { bits }}{\text { Joule }}
$$

Here $M$ is the total number of bits per frame, $L$ is the number of information bits per frame, and $L<M . R$ is the information rate in bits/sec. $\gamma_{j}$ is the SINR at terminal $j$, defined in Equation (3-7) in terms of channel bandwidth $W$, AWGN noise power at the receiver $\sigma^{2}$, and path gains from mobile to base stations $\left\{h_{j}\right\}$, for the uplink of a CDMA system.

$$
\gamma_{j}=\frac{W}{R} \frac{h_{j} p_{j}}{\sum_{i \neq j} h_{i} p_{i}+\sigma^{2}}
$$

The efficiency function $f(\gamma)$ is related but not identical to the probability of correctly decoding a frame and is defined in terms of the bit error rate (BER) $P_{e}$, as follows.

$$
f(\gamma)=\left(1-2 P_{e}(\gamma)\right)^{M}
$$

Note from Equation (3-6) that the utility function, illustrated in Figure 3-4, monotonically increases with SIR, and monotonically decreases with power. It thus serves as an intuitive, if not exact, representation of a user's perception of quality of service (QoS). In addition, it exhibits several desirable mathematical properties [49]. These properties are sufficient to verify the existence of NE in the NPG using Theorem 3-2. Additionally, all terminals are shown to achieve the same SINR in NE. The equilibrium of the NPG is then shown to be unique, since its best-response function is found to be a standard interference function [52]. However, the NE turns out to be Pareto inefficient (Definition 3-12), which is proved by noting the improvement in utilities of all terminals when the powers of a subset of $N$ are scaled down by a factor $0<\mu \leq 1$. 


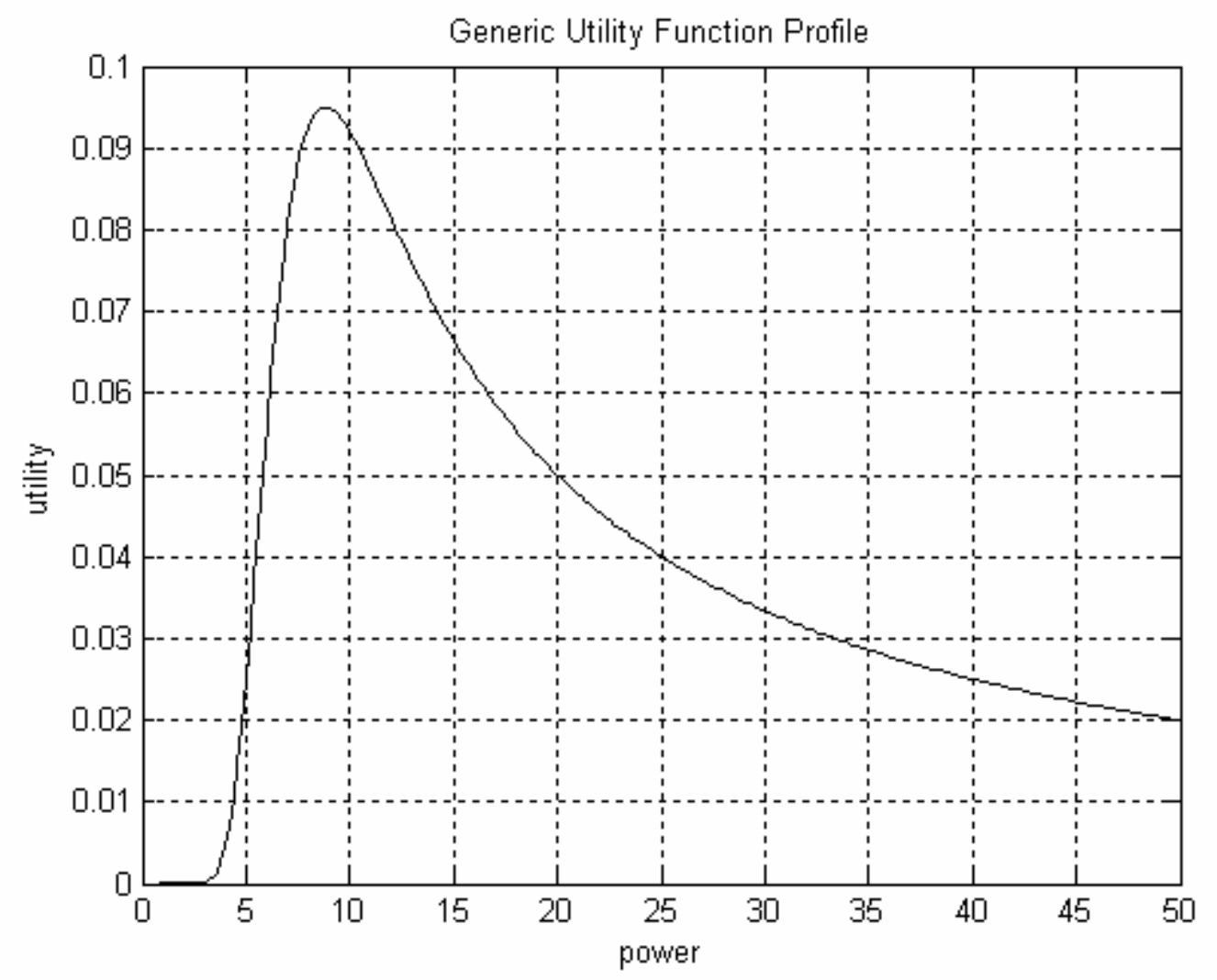

Figure 3-4 Shape of utility function in [51]

To bring about a Pareto improvement in the NE of the NPG, the authors of [51] consider pricing. This idea has its origins in economics literature (taxation) and has been applied to computer networks [54]. The pricing function selected by the authors is linear in $p_{j}$. The new NPG with pricing (NPGP) differs from the NPG in the formulation of its utility function, as stated in Equation (3-9).

$$
u_{j}^{c}\left(p_{j}, p_{-j}\right)=u_{j}-c \alpha_{j} p_{j}
$$

It turns out that in the presence of a pricing function $c \alpha_{j} p_{j}$, it is no longer possible to verify the existence of a NE in NPGP using Theorem 3-2. The authors resort to the theory of supermodular games [55], specifically those aspects that relate to supermodular games with exogenous parameters. Here, pricing factor $c$ is an exogenous parameter. Supermodular games possess a highly desirable property: they always have NE. These equilibria are usually not unique; they belong to a set called the Nash set that is bounded from above and below. The presence of the exogenous parameter requires a modification of the action sets $P_{j}$ in order for the NPGP to satisfy the conditions imposed by the definition of a supermodular game. Note that although the original formulation of the NPGP cannot be guaranteed to possess NE, it does not preclude the existence of NE in that game. 
To discover the power vector that represents an equilibrium of the modified NPGP, the authors of [51] propose a distributed and asynchronous algorithm that executes at the terminal. This algorithm uses the minimum power vector of the modified action space as an initial approximation, and is shown to generate a sequence of powers that converge to the lower bound of the Nash set. This is shown to be the Pareto-optimal equilibrium of the NPGP. At equilibrium, the SINRs are no longer equal; they tend to be higher for users in more favorable channel conditions. Thus, in general, this NE will not be a social optimum, i.e., it will not be the optimum solution of a social objective function of the form $\sum_{j \in N} \beta_{j} u_{j}$, where $\beta_{j}$ is a positive constant. A pricing function that achieves the social optimum is presented in Equation (3-10).

$$
c_{i}(\mathbf{p})=\left(-1 / \beta_{i}\right) \sum_{j=1, j \neq i}^{N} \beta_{j} u_{j}(\mathbf{p})
$$

Obviously, this pricing function is virtually impossible to realize in a distributed manner. The same work [51] investigates, through simulation, the best choice of pricing factor $c$. Further work on the optimal pricing factor selection is presented in [56], with an intent to simultaneously maximize $\sum_{j \in N} \beta_{j} u_{j}, \beta_{j}>0$, at equilibrium.

In a follow-up to [51], the NPG is extended to a multi-cell CDMA network [57]. Assuming that each terminal can only communicate with a single base station, implying that soft handoff is not permitted, the optimal assignment of terminals to base stations is an added dimension to the authors' earlier problem. Two assignment strategies are chosen. The first, based on maximum received signal strength (MRSS) simply assigns a terminal to the closest base station. This reduces the original two-dimensional problem to a single-dimensional problem in the powers only, similar in form to the single-cell system considered in [51]. Pricing may be global, implying the assignment of a single pricing factor to all base stations, or local, where each base station selects its own pricing factor. The choice of a local pricing factor that is proportional to the number of terminals assigned to a base station is investigated.

The second assignment strategy arises from the joint maximization of $u_{a_{j} j}\left(p_{j}, p_{-j}\right)$ over the assignment $a_{j}$ and power $p_{j}$. It is shown that this reduces to choosing

$$
a_{j^{\prime}}=\arg \max _{a_{j}} \gamma_{a_{j} j}
$$

This is equivalent to assigning a terminal to the base station at which its SINR is maximized (MSIR). In the absence of pricing, a unique equilibrium SINR is shown to exist. Pricing results in a different base station assignment since the SINR set is now heterogeneous.

There have been similar efforts in literature to the one described in [51], differing from each other primarily in their choices of utility function models. The nature of the results presented in most work is contingent on the choice of utility function. It is important for this function to be intuitive, accurate and mathematically tractable. In [58], the uplink of a multi-rate CDMA system is once again studied. The authors use the information- 
theoretic capacity formula [59] for a binary input Gaussian output (BIGO) channel, or a binary symmetric channel (BSC), as part of the utility function. This formula, denoted by $f(x)$, is graphically illustrated in Figure 3-5, where $x$ is the SINR. Scaling $f(x)$ by the signal transmission rate of the source $R_{i}$, we obtain an expression for the throughput of user $i$. This choice of utility function is shown to possess more suitable mathematical properties than the one proposed in [51] (see Equation (3-6)).

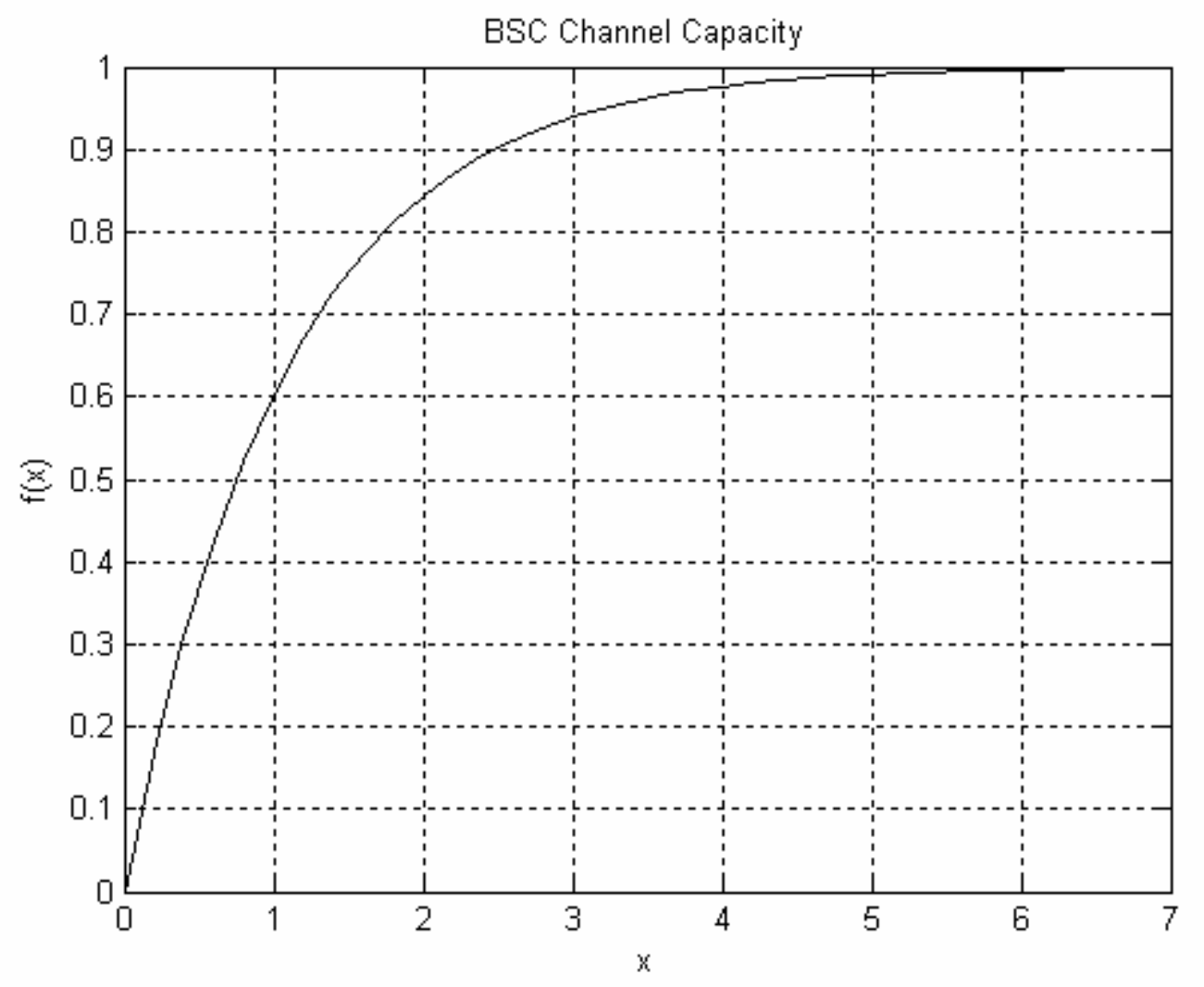

Figure 3-5 Information-theoretic capacity $[f(x)]$ versus SINR $[x]$ of a BSC channel [58]

In the absence of pricing, there will be some users that reach their received power ceilings, and this solution is shown to be Pareto-optimal. The reason for this is that $f$ is monotonically increasing in $x$. However, this kind of power saturation is inefficient from a system perspective. The solution, once again, is to introduce pricing. The choice of pricing function for a terminal $i$ is proportional to the fraction of total received power at the base station, due to $i$. This accurately describes the contribution of $i$ to the interference at the base station, and penalizes it proportionately. The modified utility function has the following form.

$$
v_{i}\left(Q_{i}, Q_{-i}\right)=R_{i} f\left(x_{i}\right)-\frac{\lambda Q_{i}}{Q_{i}+I_{i}}
$$

Here $\lambda$ is the pricing factor, $Q_{i}$ is user $i$ 's received power and $I_{i}$ is the interference caused to user $i$ by the transmissions of other users. Hence, the denominator of the pricing function is the total received power at the base station. A unique $\operatorname{NE} \mathbf{Q}(\lambda)$ is shown to exist in this game, for a reasonable choice of $\lambda$. The solution $\mathbf{Q}\left(\lambda^{*}\right)$ for a particular 
value of $\lambda=\lambda^{*}$ is claimed to be Pareto-optimal for the game without pricing. This solution once again results in some users transmitting at maximum power. The author's then claim that such a solution will maximize system throughput $\sum_{j=1}^{N} R_{j} f\left(x_{j}\right)$, for a given total received power $\sum_{j=1}^{N} Q_{j}$, since system throughput is a monotonically increasing function of SINR. However, their proof in this case assumes that the solution $\mathbf{Q}\left(\lambda^{*}\right)$ is Pareto-optimal for the game with pricing. This assumption is clearly in contradiction with the previous result for the game without pricing. Hence, it is not clear whether $\mathbf{Q}\left(\lambda^{*}\right)$ could maximize system throughput.

In [60], the utility function is the difference of a linear pricing function and a term that is proportional $-\alpha_{i}$ being the proportionality factor - to Shannon capacity, as shown in Equation (3-13).

$$
J_{i}\left(p_{i}, p_{-i}\right)=\lambda_{i} p_{i}-\alpha_{i} \ln \left(1+\gamma_{i}\right)
$$

Here, due to the formulation of the utility function, the objective is to minimize $J_{i}$. Using the standard first-order derivative condition for minima, the authors establish conditions for the existence of a unique NE in which all but a certain number of users have non-zero powers. A closed-form solution for the non-zero equilibrium power levels is also presented. The work also features two iterative algorithms for updating the power. In [61], the following form of utility function, where $a$ and $b$ are positive constants, and the rest of the terms have the same meaning as before, is shown to result in the existence of a unique NE.

$$
u_{i}\left(p_{i}, p_{-i}\right)=a\left(\frac{\gamma_{i}}{b+\gamma_{i}}\right)-\lambda_{i} p_{i}
$$

As we have already seen, it is common to encounter the use of utility functions that are monotonically increasing in SINR, priced by a term proportional to power. In our work, we employ a sigmoid model for throughput. This model, as we shall demonstrate in Chapter 4, is the closest approximation to typical simulated throughput versus SINR profiles. An intuitive and analytically tractable utility function based on this model is derived in [62]. The sigmoid function is an S-shaped curve described by Equation (3-15).

$$
f(x)=\frac{A}{1+e^{-\lambda(x-\delta)}}
$$

Here $A$ is the peak value of the sigmoid function, $\delta$ is the abscissa of the point of maximum slope, such that $f(\delta)=A / 2$, and $\lambda$ is a steepness factor. Curves of this nature can be often encountered in the neural networks literature. The sigmoid function is plotted in Figure 3-6, with $A=1, \delta=0$, and $\lambda=1$. 


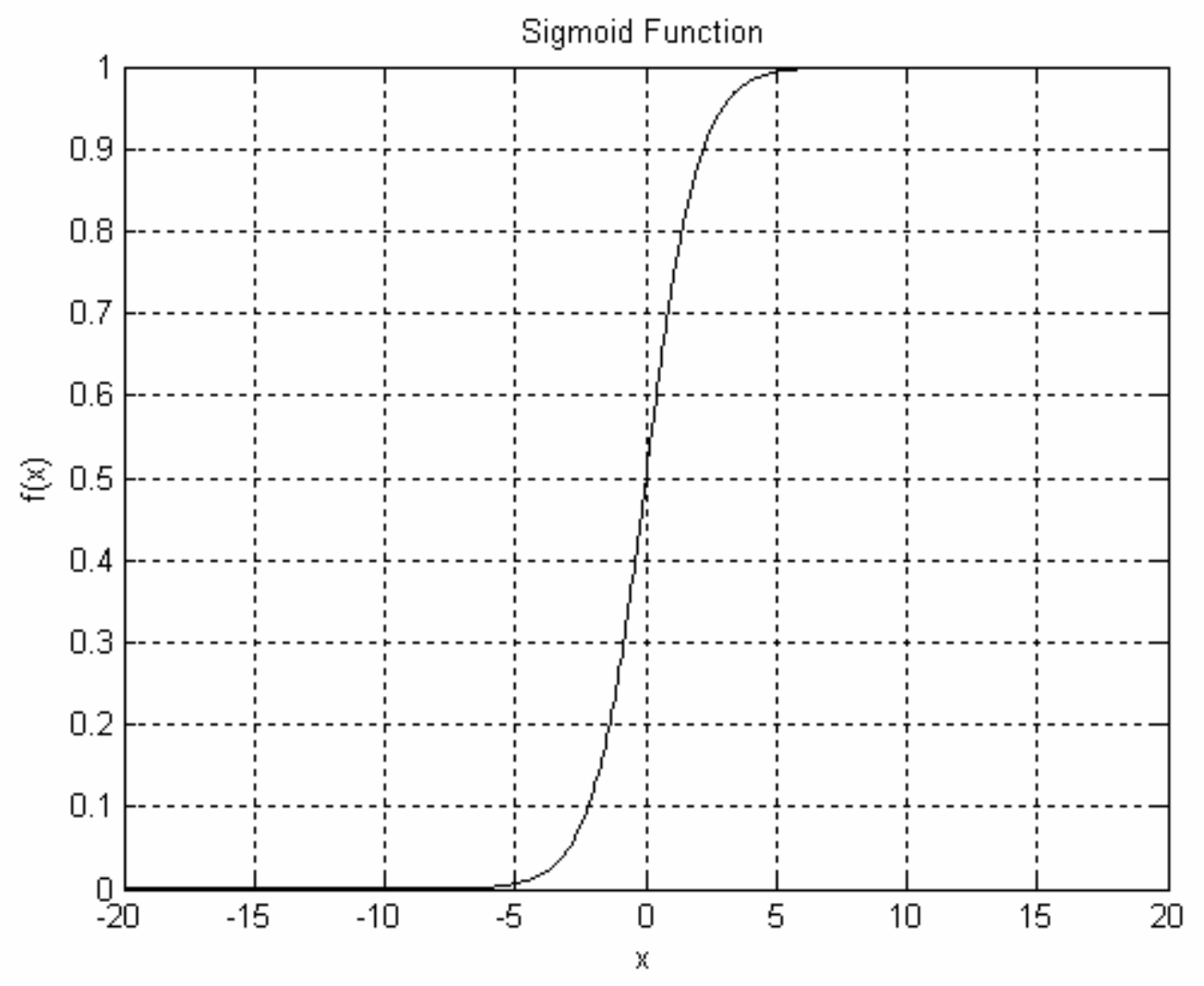

Figure 3-6 Sigmoid function with $A=1, \delta=0, \lambda=1$

The sigmoid model is employed in the utility-based power control (UBPC) scheme presented in [63]. While not strictly employing game theory, the utility-based approach bears several similarities to a rigorous game-theoretic approach. The belief that user satisfaction will depend on both QoS and power consumption forms the foundation of this work. The paper compares the merits of using UBPC to traditional distributed power control (DPC) [18] and justifies that the divergence that occurs in DPC when the system is infeasible is not an issue in UBPC. The reason offered is that the hard SINR target requirement in DPC is "softened" in UBPC. In other words, an unachievable SINR target is reduced, or, in extreme cases, a user is turned off until system feasibility is attained. A noteworthy feature of this work is the detailed analysis conducted on the choice of sigmoid function parameters for different scenarios, and the bounds imposed on the slope of the linear pricing function. Extensions such as adapting the pricing parameter as either a function of channel conditions, or to mitigate the near-far unfairness that is inherently produced by UBPC, are suggested. Integration of UBPC with admission control, dynamic base-station assignment and link adaptation are further recommendations. In fact, in Chapter 4 we address joint link adaptation and power control. In summary, the merits of [63] are applicable to other utility functions. Thus, it serves as a convincing rationale for the usefulness of utility-based approaches to power control. Another related application of this approach is the distributed multi-user power control scheme for Digital Subscriber Line (DSL) technology presented in [64]. 
Joint transmission rate adaptation and power control is the subject of [65]. This work utilizes the same form of the utility function without pricing, as proposed in [51] (see Equation (3-6)), with the distinction that the transmission rate and power are both controllable. Using first-order optimality conditions, a solution that achieves the same SINR for all users is obtained. The optimality conditions result in $N$ equations with $2 N$ variables, consisting of $N$ power-rate pairs. Therefore, according to Equation (3-7), there are infinite combinations of power and rate that accomplish this SINR. The authors assume that the rates must be fixed, and identical for all users. To maximize the processing gain, the maximum of the minimum rates of all users is selected. An intuitive justification for the multiplicity of solutions in this problem is that the ratio of power $(P)$ and transmission rate $(R)$ is the energy per bit, and infinite combinations of power and rate can solve the equation $P / R=\mathrm{E}$, where $\mathrm{E}$ is a constant. The key point is that this scheme does not classify as true link adaptation. In true link adaptation, the FER probability should be a function of rate. Then, even for the same energy per bit, different rates result in different utilities. In the following chapter, we address precisely this problem.

\subsection{Summary}

Game theory is considered to be an unfamiliar topic to the wireless engineer. Thus, the presentation in this chapter began at a fundamental level, providing a number of textbook references for further reading. Core mathematical concepts from set theory and game theory were stated in Section 3.5, the only mathematical section of this chapter. These are necessary for understanding the content of Chapter 4. The next section presented an exhaustive number of examples of game-theoretic applications to power control. A brief rationale for applying game theory to power control problems was also mentioned. It is worth contrasting game-theoretic approaches to the approaches presented in Section 2.4, which we refer to as "system-theoretic" approaches. The current chapter sets the stage for the main contribution of this thesis, i.e. our own game-theoretic technique for solving the link adaptation problem, which forms the subject of Chapter 4. 


\section{A Game-theoretic Approach to Link Adaptation $^{5}$}

In our literature review in Chapter 3, we summarized several game-theoretic approaches to the power control problem for data networks. We also observed that these approaches can be extended and generalized to incorporate schemes with multiple code rates and modulation schemes. To date, there has not been sufficient literature on this subject. In this chapter, we develop a utility-based model that is a function of two variables transmitter power and code rate. Note the distinction from the typical utility-model of the previous chapter in which the only variable was power. By keeping the code rates fixed our model collapses into a function of solely power. Thus, we claim that our model is a generalization of the models found in Chapter 3.

\subsection{Link Adaptation}

Link adaptation is a well-understood technique for exploiting the variability of a fading mobile radio channel [66]-[70]. Other terms applied to this technique are adaptive modulation and coding, rate adaptation, and variable-rate modulation. Traditional communication techniques employ fixed modulation and coding schemes. These techniques may use power control to adapt their transmit power to achieve a particular performance target in terms of bit error rate (BER). The spectral efficiency of a fixed modulation and coding scheme remains fixed. However, it is possible to achieve a greater average spectral efficiency by varying a performance-impacting link characteristic such as the modulation or coding scheme. This is essentially what link adaptation accomplishes. A generic link adaptation scheme works by forming a channel quality estimate [71] at the receiver, which is fed back to the transmitter. Assuming that the channel remains static during this period, the transmitter chooses the value of the adaptive link characteristic that maximizes the spectral efficiency whilst meeting the BER constraint. Since the transmitter power will be a function of the choice of modulation and coding, power control and link adaptation are related. It is, therefore, sensible to consider a scheme in which these two functions are jointly executed. This chapter focuses on the development of one such scheme using a game-theoretic approach.

\footnotetext{
${ }^{5}$ This chapter utilizes the mathematical concepts and notation presented in Section 3.5. It is recommended that the reader familiarize himself/herself with the contents of this section.
} 


\subsection{Game-theoretic Formulation}

\subsubsection{System Model}

We consider a set $T=\left\{T_{1}, T_{2}, \ldots, T_{N}\right\}$ of $N$ co-channel links in a cellular data system. A single transmitter-receiver pair is referred to as a link. For the purpose of our analysis, we consider a "traditional" system model of the form described in Section 2.1 (see Figure $2-1)$. Note that although this system model is typical for non-CDMA systems, the extension to CDMA systems is fairly straightforward. To construct a model that is as general as possible, we do not distinguish between the uplink and downlink. The link gain matrix of the system, described in Section 2.1, is assumed to remain constant throughout the analysis. The channel can be assumed to be static for the period of convergence of the algorithms that execute on each link $i \in T$.

It is assumed that each link $i \in T$ can adapt a particular link characteristic or parameter $r_{i}$, referred to as the adaptable link parameter (ALP). We require that $r_{i}$ be chosen from a finite set $R=\left\{r^{1}, r^{2}, \ldots, r^{|R|}\right\}$. The number of different variations of this parameter is the cardinality $|R|$ of set $R$. In addition, each link $i \in T$ is power controlled, i.e., it can adjust its power $P_{i}$. The powers are selected from a set $P=\left\{p: p \in\left[P_{\min }, P_{\max }\right]\right\}$. Thus, $P_{i}$ is lower-bounded by $P_{\min }$ and upper-bounded by $P_{\max }$.

We specify that the information that is communicated across each link is non-voice, besteffort type data. There are no pre-assigned priorities among different classes of traffic, which implies that all links can expect identical service, all other parameters being equal. The impact of the upper layers of the protocol stack (above the medium access control (MAC) layer) on performance is neglected. For this type of data service, a valid measure of link performance is the effective (net) link throughput $L$. The link throughput is essentially the "goodput." If $R_{b}$ is the transmission rate in bits/sec on the link $i \in T$ and FER is the frame error rate, as a function of Signal to Interference and Noise Ratio (SINR) $\gamma_{i}$, then we have the following expression for net throughput on link $i$, which we shall simply refer to as throughput henceforth.

$$
L_{i}=R_{b}\left(1-F E R\left(\gamma_{i}, r_{i}\right)\right)
$$

It is obvious that one objective of each link is the maximization of its throughput. However, that this is at cross-purposes with the objectives of other links is evident from Equation (4-1). The FER is a monotonically decreasing function of $\gamma_{i}$. Hence, as $\gamma_{i}$ increases, link throughput increases. We observe that the SINR of link $i$ in Equation (4-2) increases in direct proportion to its own power (numerator), but in inverse proportion to the interference (denominator). Thus, it is clear that increasing the throughput of a particular link degrades the throughput of the others. 


$$
\gamma_{i}=\frac{G_{i i} P_{i}}{\sum_{j \neq i} G_{i j} P_{j}+n_{i}}
$$

In addition, we note from Equation (4-1) that throughput is also a function of the ALP $r_{i}$. It is, therefore, possible to model this situation as a non-cooperative game, which we conveniently name the Link Adaptation Game (LAG). To construct a game-theoretic model for this situation using Definition 3-9, we must identify players, their actions and define a preference relation or utility function for each player.

\subsubsection{Players}

An obvious candidate for the set of players is $T$, the set of co-channel links. Henceforth, we use the term player and link interchangeably.

\subsubsection{Action Space}

We define the action selected by any player $i \in T$ as the pair $\left(P_{i}, r_{i}\right)$, where $P_{i} \in P$ is the power of player $i$ and $r_{i} \in R$ is the ALP of player $i$. A power vector $\mathbf{P}$ and an ALP vector $\mathbf{r}$ are defined as $\mathbf{P}=\left(P_{i}\right)_{i \in T}$ and $\mathbf{r}=\left(r_{i}\right)_{i \in T}$. The action space $A_{i}$ of any player is defined by $A_{i}=P \times R$. The action space of the game is $A=\times\left(A_{i}\right)_{i \in T}$.

\subsubsection{Utility Function}

Equation (4-1) and the discussion accompanying it suggest that the expression for throughput merits inclusion as a component in an expression for link utility. However, due to the non-cooperative nature of this game, it is easy to see that in an attempt to maximize throughput at any cost, each link is likely to consume maximum power, since throughput increases monotonically with power. This will also serve to create excessive interference, leading instead to performance degradation, since throughput decreases monotonically with interference. In the case of the uplink, there is the additional consideration of battery life, since power conservation is of paramount importance to power-limited handsets. Similar observations ${ }^{6}$ have been made in other game-theoretic analyses of power control schemes, such as those in [49] and [51]. These schemes were described in Chapter 3. The suggested solution to this problem in these instances was to introduce pricing. We adopt the same approach to penalize the use of excessive power. This strategy also induces a degree of co-operation amongst links, and can be imagined to bring about an improvement in system performance. The utility function of player $i \in T$ is defined as follows.

$$
U_{i}\left(\mathbf{P}, r_{i}\right)=L_{i}\left(\gamma_{i}, r_{i}\right)-C_{i}\left(P_{i}\right)
$$

\footnotetext{
${ }^{6}$ The analogy with the famous "Tragedy of the Commons" essay [Garrett Hardin, Science, Dec 1968] is illuminating. In this instance, the "commons" refers to the shared wireless medium. Overuse of wireless resources can be discouraged by introducing a usage cost.
} 
Here, the throughput function $L$ is described by Equation (4-1) and $C_{i}\left(P_{i}\right)$ is called the penalty or pricing or cost function. It is a function of only the power of player $i \in T$ and is defined in Equation (4-4).

$$
C_{i}\left(P_{i}\right)=K P_{i}^{q}
$$

The parameters of the cost function, $K$ and $q$, are positive constants. To restrict the range of our variables we set the weighting or scaling parameter $K$ to 1 , and permit variation in index or exponent, $q$. When $q=1$ we have linear pricing that is commonly used in the literature. However, we can shape the penalty function by using $q>1$, such that links are penalized more severely for consuming power, than they would be under linear pricing. Alternatively, if power is not critical, a more lenient $q<1$ might be preferable. The penalty function profiles for different values of $q$ are depicted in Figure 4-1.

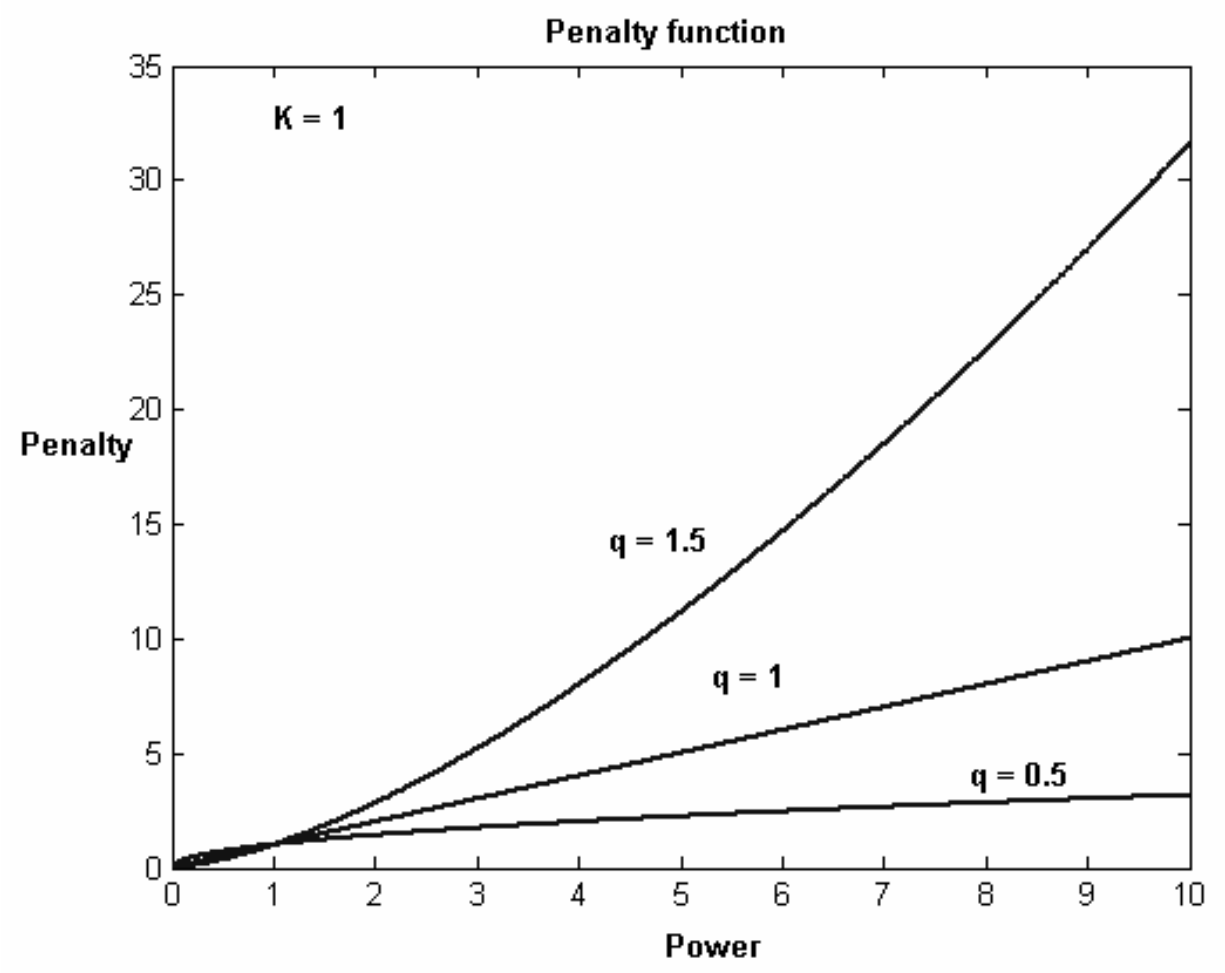

Figure 4-1 Penalty function as a function of power for different indices $q$

In Equation (3-15) and Figure 3-6, the sigmoid function was introduced. This model has been shown in [62] and [63] to be capable of accurately approximating the throughput versus SINR profile of a wireless link. The sigmoid function of SINR is presented in Equation (4-5).

$$
L_{i}\left(\gamma_{i}, r_{i}\right)=\frac{\alpha\left(r_{i}\right)}{1+e^{-\lambda\left(r_{i}\right)\left[\gamma_{i}-\delta\left(r_{i}\right)\right]}}
$$

Here, the SINR of $i \in T$ in $d B$ is defined in Equation (4-6). 


$$
\gamma_{i}=10 \log _{10}\left(\frac{G_{i i} P_{i}}{\sum_{j \neq i}^{N} G_{i j} P_{j}+n_{i}}\right)
$$

$\alpha$ is the peak value of the sigmoid function, $\delta$ is the abscissa of the point of maximum slope of the sigmoid, such that $L\left(\delta, r_{i}\right)=\alpha\left(r_{i}\right) / 2$, and $\lambda$ is the steepness factor. Figure $1-2$, justifies the need for the parameters of the sigmoid function, namely $\alpha, \lambda$, and $\delta$, to be functions of the ALP, which, in Figure 1-2, is the code rate. We demonstrate in the Section 4.2.5, how the parameters of the sigmoid function can be chosen to closely fit the simulated throughput-SINR profile of a given link.

\subsubsection{Example: GPRS}

The preceding development is rather general. However, in all our simulations and results we have exemplified our work through a typical GPRS network. GPRS supports link adaptation [6]. In GPRS, the modulation scheme is Gaussian minimum shift keying (GMSK), but four options for code rate, referred to as coding schemes (CS), are specified. Thus, the ALP of a GPRS network is its code rate. The key features of the GPRS coding schemes are summarized in Table 4-1 [73].

Table 4-1 GPRS Coding Schemes [73]

\begin{tabular}{|c|c|c|c|}
\hline Coding Scheme & Modulation & Code Rate & Data rate/ Time slot \\
\hline CS-I & GMSK & 0.49 & $9.05 \mathrm{kbps}$ \\
\hline CS-II & GMSK & 0.64 & $13.4 \mathrm{kbps}$ \\
\hline CS-III & GMSK & 0.73 & $15.6 \mathrm{kbps}$ \\
\hline CS-IV & GMSK & 1 & $21.4 \mathrm{kbps}$ \\
\hline
\end{tabular}

Simulation curves for throughput versus SINR are reproduced from [73] and shown in Figure 4-2 for a particular channel model (TU-50), with ideal frequency hopping, for all the rates listed in Table 4-1.

The sets of parameters of the sigmoid model in Equation (4-5) that best approximate the simulation curves of Figure 4-2 are selected by computer-aided search and summarized in Table 4-2.

Table 4-2 Parameters of Sigmoid Models for Throughput vs. SINR curves

\begin{tabular}{|l|l|l|l|}
\hline CS & $\boldsymbol{\alpha} \mathbf{k b p s}$ & $\boldsymbol{\lambda}$ & $\boldsymbol{\delta} \mathbf{~ d B}$ \\
\hline CS-1 & 7.36 & 0.272 & 4.75 \\
\hline CS-2 & 10.52 & 0.256 & 8.250 \\
\hline CS-3 & 11.88 & 0.256 & 9.5 \\
\hline CS-4 & 14.36 & 0.231 & 15 \\
\hline
\end{tabular}




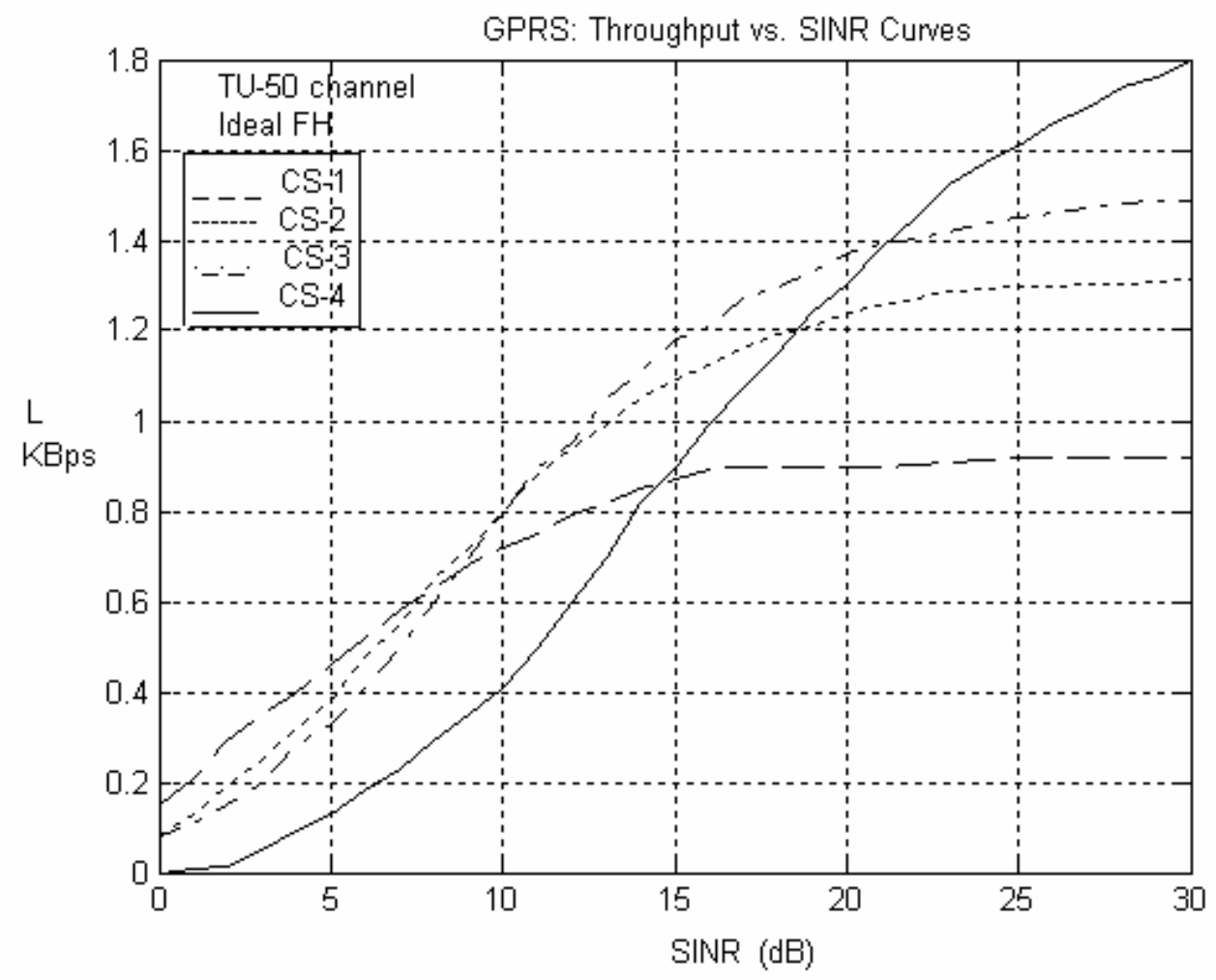

Figure 4-2 Example GPRS throughput versus SINR simulation curves [73] showing comparison of performance using different code rates. The y-axis is in units of kilobytes per second (KBps). (TU-50: Typical Urban fading channel, mobile speed $=50 \mathrm{~km} / \mathrm{hr}$. FH $=$ Frequency Hopping.)

A sample population of $n$ coordinate pairs $(\mathbf{X}, \mathbf{Y})$ on the simulation curves, selected at regular $\mathrm{dB}$ intervals of $\mathrm{SINR}$, is fed as raw data to the following procedure for deriving the sigmoid approximation parameters, given a particular coding scheme.

1. The parameter $\alpha$ is the peak throughput (since $\alpha=\lim _{\gamma_{i} \rightarrow \infty} L_{i}$ ) and is easily found by searching for the peak of the ordinates of the sample population, as stated in Equation (4-7).

$$
\alpha=\max \mathbf{Y}
$$

2. In the next step, the knowledge that $\delta$ is the abscissa of the point of maximum slope is exploited in order to arrive at a first approximation to $\delta$. This step is equivalent to first finding the approximate index $i_{\delta}$ of maximum slope, as shown in Equation (4-8).

$$
i_{\delta}=\arg \max _{i=\{1,2, \ldots, n-1\}}\left(\frac{Y_{i+1}-Y_{i}}{X_{i+1}-X_{i}}\right)
$$

Then, as shown in Equation (4-9), the mid-point of the corresponding abscissa $X_{i_{\delta}}$ and its immediate higher neighbor in $\mathbf{X}$ forms the first approximation to $\delta$. 


$$
\delta^{\prime}=\frac{X_{i_{\delta}}+X_{i_{\delta}+1}}{2}
$$

Note that the expression in the parentheses in Equation (4-8) is always positive due to the monotonic nature of the throughput-SINR profile. The paucity of points in the sample population introduces inaccuracy in the calculation of $\delta$ and motivates the next step.

3. A joint search for $\delta$ and $\lambda$ is now conducted. A range of values $\tilde{\lambda} \in[0,1]$ is used as the search space for $\lambda$ and a small search window (approximately $\Delta=10 \mathrm{~dB}$ ) in the neighborhood of $\delta^{\prime}$, denoted by $\tilde{\delta} \in\left[\delta^{\prime}-\Delta / 2, \delta^{\prime}+\Delta / 2\right]$, is used as the range for $\delta$. The $(\tilde{\lambda}, \tilde{\delta})$ pair that minimizes the mean-square error $\sigma$ in Equation (4-10) is assigned to $(\lambda, \delta)$.

$$
\sigma=\frac{1}{n} \sum_{i=1}^{n}\left(Y_{i}-\frac{\alpha}{1+e^{-\tilde{\lambda}\left(X_{i}-\tilde{\delta}\right)}}\right)^{2}
$$

The result of this curve fitting process for CS-3 is illustrated in Figure 4-3 to demonstrate the efficacy of our parameter approximation algorithm. Perhaps more importantly, it also supports our choice of the sigmoid function for throughput.

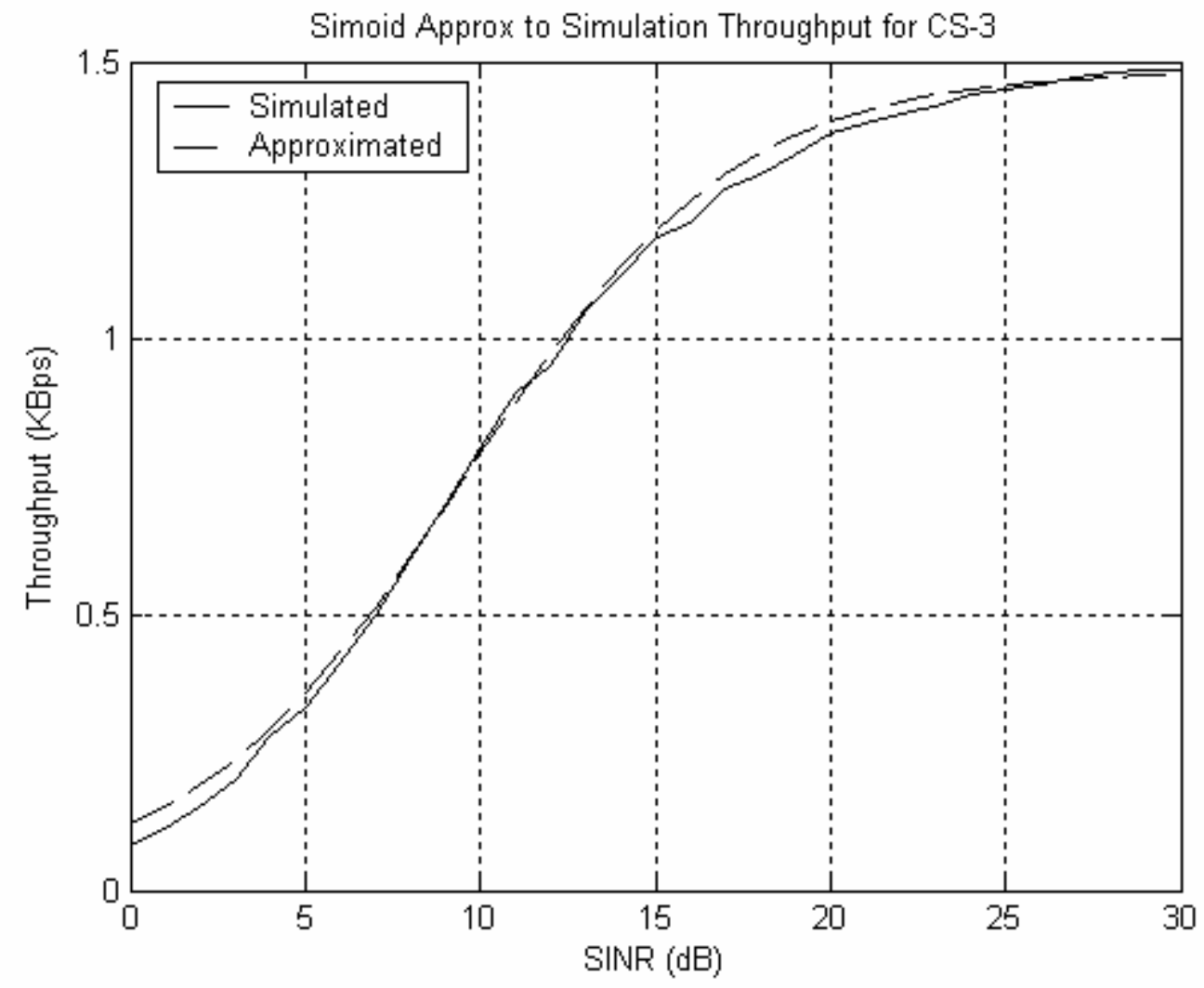

Figure 4-3 Results of sigmoidal curve-fitting to simulation data for CS-3. The dashed curve is the approximation 
In Figure 4-4, the utility function for a player involved in a 2-player game is shown. The $\mathrm{x}$-axis of the figure is the power and the y-axis, the utility. The power of the second player is 2 units. The link gains are $G_{11}=1$, and $G_{12}=0.01$. It is assumed that the signal to noise ratio $E_{b} / N_{o}$, referenced to a signal energy of 1 unit, is $20 \mathrm{~dB}$. Thus, the noise power works out to be 0.005 units. The penalty function parameters are $K=1$ and $q=2$. The utility function profiles seem to indicate the existence of a unique maximum. In the next section, we formally assess some significant properties of the LAG and its utility function.

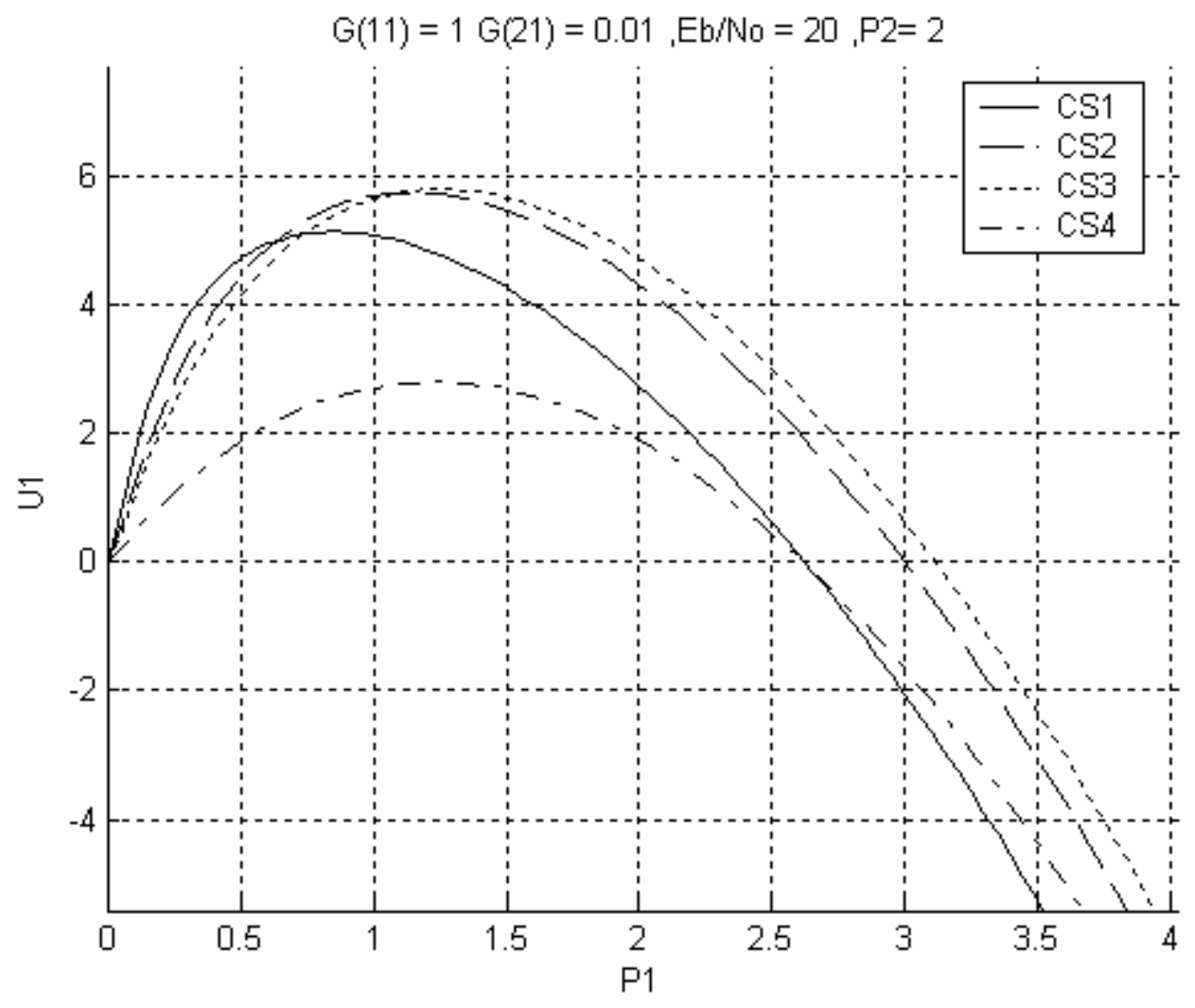

Figure 4-4 Utility function in a 2-player game, as a function of power, for different rates

\subsection{Properties of the Game}

In this section, we prove some useful properties of the game LAG, which will subsequently be used in establishing some important results.

\section{Lemma 4-1}

The action space $A_{i}$ of player $i \in T$ is non-empty and compact. 


\section{Proof:}

By definition, the action set of player $i \in T$ is non-empty. By definition (see Section 4.2.1), $P$ is closed and since $P_{\max }<\infty$, it is also bounded. The set of rates $R$ is finite; hence, $R$ is also closed and bounded. Therefore, every ordered pair $\left(P_{i}, r_{i}\right)$ belongs to a space $A_{i}$ that is closed and bounded. Therefore, by Definition 3-3, $A_{i}$ must be compact. This completes the proof.

\section{Lemma 4-2}

$A_{i}$ is not a convex set. However, under the condition that $r_{i}$ is fixed, $A_{i}$ is a convex set.

\section{Proof:}

To prove that $A_{i}$ is not convex, we simply choose two points, $A_{1}=\left(P_{1}, r^{1}\right)$ and $A_{2}=\left(P_{1}, r^{2}\right)$, that have the same power component. A line connecting these points consists of only two points, $A_{1}$ and $A_{2}$ themselves, that belong to $A_{i}$. The intervening points on this line do not belong to $A_{i}$. Hence, by Definition 3-4, the set $A_{i}$ is not convex.

However, suppose the ALP is fixed at, say $r^{1}$. We note that a convex combination $\lambda^{\prime} A_{1}+\left(1-\lambda^{\prime}\right) A_{2}, \lambda^{\prime} \in[0,1]$ - where $A_{1}=\left(P_{1}, r^{1}\right)$ and $A_{2}=\left(P_{2}, r^{1}\right)$ are any two arbitrarily selected actions in $A_{i}$, such that $P_{\min } \leq P_{1}<P_{2} \leq P_{\max }-$ belongs to the set $P^{\prime}=\left\{p: p \in\left[P_{1}, P_{2}\right]\right\}$. Clearly, $P^{\prime} \subseteq P$. Hence, applying Definition $3-4$, the set $A_{i}$ is convex when $r_{i}$ is fixed and the proof is complete.

Figure 4-5 depicts the action space of a player from the GPRS example introduced in Section 4.2.5. It is easy to see graphically that $A_{i}$ is not convex, but $P$ is. Therefore, if we maintain $r_{i}$ fixed, it follows that $A_{i}$ is convex.

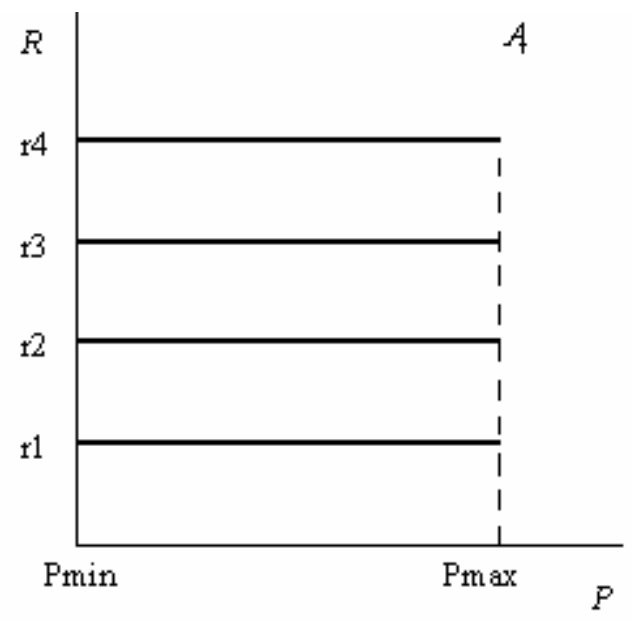

Figure 4-5 $A_{i}$ : Action space of player $i \in T$ 
Due to Lemma 4-2, the existence of a NE in game LAG is not guaranteed. However, by maintaining the ALP $r_{i}$ at a fixed value, we show that it is possible to show the existence of a NE. To this end, we present the following two lemmas. The first, Lemma 4-3, shows that the utility function of each player is continuous in the action space of the game $A$. In the second, Lemma 4-4, the utility function is shown to be quasi-concave in the power of player $i$.

\section{Lemma 4-3}

$U_{i}$ is continuously differentiable on $A$, when $\mathbf{r}$ is fixed. Hence, $U_{i}$ is continuous on A.

\section{Proof:}

Since the derivative of $U_{i}$ is undefined w.r.t. $r_{i}$, the ALP vector $\mathbf{r}$ is fixed. Then, for $U_{i}$ to be continuously differentiable, it is sufficient to show that the first-order partial derivatives of $U_{i}$ w.r.t. the components of power vector $\mathbf{P}$ are continuous [26]. Differentiating $U_{i}$ in Equation (4-5) w.r.t. $P_{i}$, we obtain

$$
\frac{\partial U_{i}}{\partial P_{i}}=\frac{\alpha \lambda e^{-\lambda\left(\gamma_{i}-\delta\right)}}{\left(1+e^{-\lambda\left(\gamma_{i}-\delta\right)}\right)^{2}} \cdot \frac{\partial \gamma_{i}}{\partial P_{i}}-K q P_{i}^{q-1}
$$

Inserting into Equation (4-11) the expression for $\gamma_{i}$ from Equation (4-6), we have after some simplification

$$
\frac{\partial U_{i}}{\partial P_{i}}=\frac{\alpha \lambda e^{-\lambda\left(\gamma_{i}-\delta\right)}}{\left(1+e^{-\lambda\left(\gamma_{i}-\delta\right)}\right)^{2}} \cdot\left(\frac{10}{\ln 10} \cdot \frac{1}{P_{i}}\right)-K q P_{i}^{q-1}
$$

Similarly, differentiating $U_{i}$ in Equation (4-5) w.r.t. $P_{j}, j \neq i$, yields

$$
\frac{\partial U_{i}}{\partial P_{j}}=\frac{\alpha \lambda e^{-\lambda\left(\gamma_{i}-\delta\right)}}{\left(1+e^{-\lambda\left(\gamma_{i}-\delta\right)}\right)^{2}} \cdot\left(\frac{-10}{\ln 10} \cdot \frac{G_{i j}}{I_{i}}\right), \quad j \neq i
$$

Here, $I_{i}$ is the interference and noise power at the receiver of link $i$. It is defined in Equation (4-14).

$$
I_{i}=\sum_{\substack{j=1, j \neq i}}^{N} G_{i j} P_{j}+n_{i}
$$

We observe that the partial derivatives in Equation (4-12) and Equation (4-13) are sums, differences, products and ratios of exponentials and polynomials. Therefore, these expressions are continuous. Hence, $U_{i}$ is continuously differentiable on $A$, which implies that $U_{i}$ is continuous on $A$ and the proof is complete.

\section{Lemma 4-4}

$U_{i}$ is quasi-concave over $A_{i}$, when $r_{i}$ is fixed. 


\section{Proof:}

Since $r_{i}$ is fixed, it remains to prove that $U_{i}$ is quasi-concave over $P_{i}$. We use graphical techniques in this proof. We consider the two-player GPRS game, described in Section 4.2.5, as an example. A series of utility function profiles for a player, say player 1, are shown in Figure 4-6. The $\mathrm{x}$-axis of the figure is the power and the y-axis the utility. Without loss of generality, the code rate of the player 1 is fixed at CS-1. The power of the second player, say player 2, is assigned in turn to each of the values in the set $\{0,3.33,6.67,10\}$ to obtain variable amounts of interference. Whether the interference is generated by one player or several players is immaterial. Hence, the two-player example is equivalent to the general $\mathrm{N}$-player case for the purpose of this proof. For each value of interference, an instance of the utility function profile of player 1 is illustrated in Figure 4-6. In this example, $P_{\min }=0$ and $P_{\max }=10$. Hence, the profiles marked by "Pmin" and "Pmax" bound the entire family of utility profiles of player 1 that are obtained by letting $P_{2}$ take every value in $\left[P_{\min }, P_{\max }\right]$. The link gains are $G_{11}=1$, and $G_{12}=0.01$. It is assumed that the signal to noise ratio $E_{b} / N_{o}$, referenced to a signal energy of 1 unit, is $100 \mathrm{~dB}$. Thus, the noise power works out to be 5e-11 units. The penalty function parameters are $K=1$, and $q=2$.

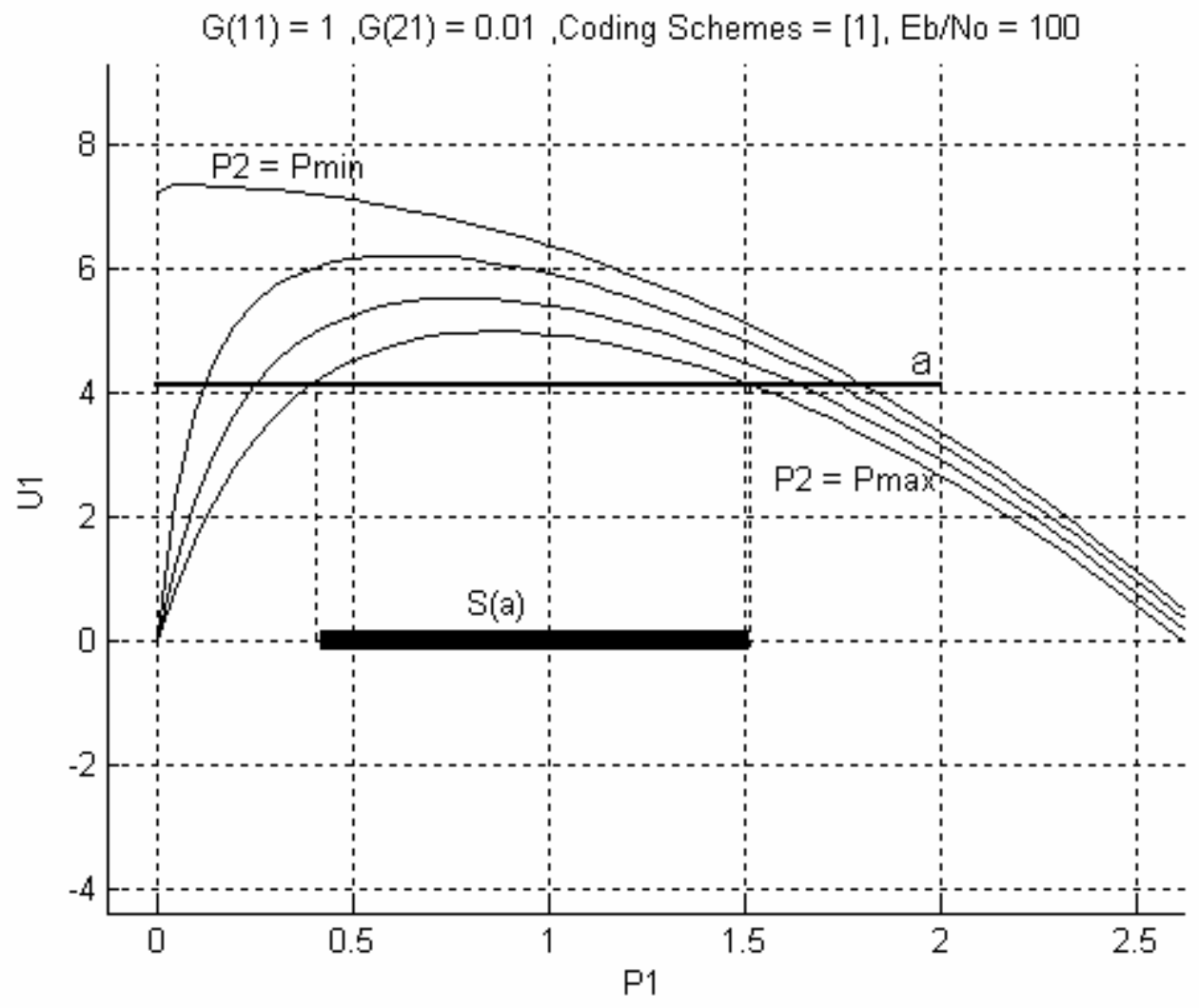

Figure 4-6 Demonstration of quasi-concavity of the utility function 
We make use of the following property of quasi-concave functions: the level-sets of quasi-concave functions are convex. This useful property is formally stated as follows [23]:

A function $f: S \rightarrow E_{1}$, where $S$ is a convex set in $E_{n}$, is said to be quasi-concave on $S$, if and only if its level set $S(a)=\{\mathbf{x} \in S: f(\mathbf{x}) \geq a\}$ is convex for each real number $a$.

In Figure 4-6, we select an arbitrary level marked "a." The level set corresponding to "a" is denoted by "S(a)." Applying Definition 3-4, we conclude that this set is convex. Therefore, using the recently defined level-set property, $U_{i}$ is quasi-concave in $P_{i}$ irrespective of the value of "a." Hence, the proof is complete.

Note that it is also possible to prove Lemma 4-4 using Definition 3-6. In fact, it is possible to apply Definition 3-7 to show that $U_{i}$ is strongly quasi-concave. This is useful in proving the existence of a unique global maximum, as shown in Proposition 4-1.

\section{Proposition 4-1}

$U_{i}$ has a unique global maximum, assuming $r_{i}$ is fixed.

\section{Proof:}

We can see in Figure 4-6 that, if we choose any open-interval $\left(P_{1}^{\prime}, P_{2}^{\prime}\right) \subset P_{i}$, any point $p$ belonging to it satisfies the condition $U_{i}(p)>\min \left\{U_{i}\left(P_{1}^{\prime}\right), U_{i}\left(P_{2}^{\prime}\right)\right\}$. Then, by Definition 3-7, $U_{i}$ is strongly quasi-concave. The proof follows from the application of the following theorem [23]:

Let $f: E_{n} \rightarrow E_{1}$ be strongly quasi-concave. Consider the problem: maximize $f(\mathbf{x})$ subject to $\mathbf{x} \in S$, where $S$ is a non-empty convex set in $E_{n}$. If $\overline{\mathbf{x}}$ is a local optimal solution, then $\overline{\mathbf{x}}$ is the unique global optimal solution.

Returning to our problem: Maximize $U_{i}\left(P_{i}\right)$ subject to $P_{i} \in P$. The set $P$ is non-empty and convex by Lemma 4-2. Further, $U_{i}$ is upper-bounded since, being a continuous image of a compact space, it is compact. Therefore, it follows that $U_{i}$ has a local maximum. Then, by the aforementioned theorem, it immediately follows that $U_{i}$ must have a unique global maximum. Hence, the proof is complete.

Finally, we prove the existence of a NE when the ALP $r_{i}$ is fixed. When the ALPs are fixed, the game LAG reduces to a power control game of the type described in Section 3.6.2. 


\section{Proposition 4-2}

A Nash Equilibrium exists in game LAG, if $\mathbf{r}$ is fixed.

Proof:

Using Lemma 4-1 to Lemma 4-4, we note that the conditions stated in Theorem 3-2 are satisfied. Hence, a NE exists in LAG, when $\mathbf{r}$ is fixed.

We have already cited examples of work where supermodularity was invoked to verify existence of NE [51]. Supermodular games always possess NE [55]. Therefore, it is worth investigating whether our game LAG is a supermodular game. The utility function of a supermodular game must demonstrate the property stated in Definition 4-1 (from Section 2.6.1 of [55]).

\section{Definition 4-1 Non-Decreasing Differences of Utility Function [55]}

If $U_{i}$ is twice differentiable on $A$, then it is said to have non-decreasing differences (NDD) in $A$, if and only if Equation (4-15) holds for all $i$ and $j$, such that $i \neq j$, and for all $a \in A$.

$$
\frac{\partial^{2} U_{i}(a)}{\partial A_{i} \partial A_{j}} \geq 0
$$

Here, $A$ is the action space of the game.

If the opposite inequality holds in Equation (4-15), then the utility function has nonincreasing differences (NID), which characterize what is known as a submodular game [55]. In fact, the following proposition shows that neither the NDD nor the NID properties hold for the game LAG.

\section{Proposition 4-3}

The game LAG is neither supermodular nor submodular.

Proof:

We show that $U_{i}$ does not satisfy the NDD property outlined in Definition 4-1. Since $U_{i}$ is not differentiable w.r.t. the ALP, we fix this parameter. This is equivalent to the special case of the game LAG in which the cardinality of the ALP set $R$ is one. If the game LAG is supermodular, then it should be so for any $|R|$. Equation (4-12) supplies the expression for $\partial U_{i} / \partial P_{i}$. This expression can be rearranged in the form shown in Equation (4-16).

$$
\frac{\partial U_{i}}{\partial P_{i}}=\left(\frac{10 \alpha \lambda}{\ln 10}\right) \cdot \frac{1}{P_{i}} \cdot\left(\frac{1}{e^{\frac{\lambda}{2}\left(\gamma_{i}-\delta\right)}+e^{-\frac{\lambda}{2}\left(\gamma_{i}-\delta\right)}}\right)^{2}-K q P_{i}^{q-1}
$$


Noticing that the term in the exponentials is the sech function, we can further simplify Equation (4-16).

$$
\frac{\partial U_{i}}{\partial P_{i}}=\left(\frac{10 \alpha \lambda}{\ln 10}\right) \cdot \frac{1}{P_{i}} \cdot \operatorname{sech}^{2}\left(\frac{\lambda}{2}\left(\gamma_{i}-\delta\right)\right)-K q P_{i}^{q-1}
$$

Differentiating Equation (4-17) w.r.t. $P_{j}, j \neq i$, we obtain after simplification

$$
\frac{\partial U_{i}}{\partial P_{i} \partial P_{j}}=\left(\frac{5 \lambda}{\ln 10}\right)^{2} \cdot\left(\frac{\alpha G_{i j}}{P_{i} I_{i}}\right) \cdot \operatorname{sech}^{2}\left(\frac{\lambda}{2}\left(\gamma_{i}-\delta\right)\right) \tanh \left(\frac{\lambda}{2}\left(\gamma_{i}-\delta\right)\right)
$$

$I_{i}$ is defined in Equation (4-14). In Equation (4-18), we notice that all terms are positive real numbers except the tanh term, which is an odd function of $\gamma_{i}-\delta$. Thus, we have the following result.

$$
\begin{array}{rlr}
\frac{\partial U_{i}}{\partial P_{i} \partial P_{j}} & \geq 0 & \\
& \gamma_{i} \geq \delta \\
& <0 & \gamma_{i}<\delta
\end{array}
$$

Applying Definition 4-1, we conclude that $U_{i}$ is neither supermodular nor submodular.

Thus far, we have not been able to confirm the existence of NE in the game LAG. However, as pointed out in Section 3.5, it is still possible for a NE to exist in this game. To discover the NE, we propose, in the following section, an algorithm based on intuitive premises.

\subsection{Solution: Algorithm LAG}

At the outset of a play of game LAG, it is not possible that a player $i \in T$ knows the complete channel gain matrix $\mathbf{G}$, and the utility functions of all other players. Hence, it is not immediately possible for a set of links in a realistic distributed environment to discover a NE immediately. However, suppose each player $i$ makes a guess, denoted by the ordered pair $\left(P_{i}, r_{i}\right)$, regarding what its equilibrium power and choice of ALP, denoted by $\left(\bar{P}_{i}, \bar{r}_{i}\right)$ should be. Then, assuming that the interference, i.e. $P_{-i}$, remains fixed while it makes a decision, player $i$ improves its guess by selecting a new combination $\left(P_{i}, r_{i}\right)$ that maximizes $U_{i}$. This results in a new approximation to $\left(\bar{P}_{i}, \bar{r}_{i}\right)$. It is logical for each player to repeat the same process of utility-maximizing unilateral deviations from the previous action, so as to obtain further refinements of the approximation to $\left(\bar{P}_{i}, \bar{r}_{i}\right)$. In game-theoretic vocabulary, the player is said to be playing a best-response. When the deviations in all players' actions become negligibly small, the algorithm can be assumed to have converged to a NE. We use the index variable $k$ to represent an iteration of the algorithm or, in general, a time index. Then, the flowchart in Figure 4-7 summarizes this algorithm, referred to as Algorithm LAG. Notice how, in the main step, we keep $r_{i}$ constant while maximizing $U_{i}$ as a function of $P_{i}$ only, and vice-versa. 


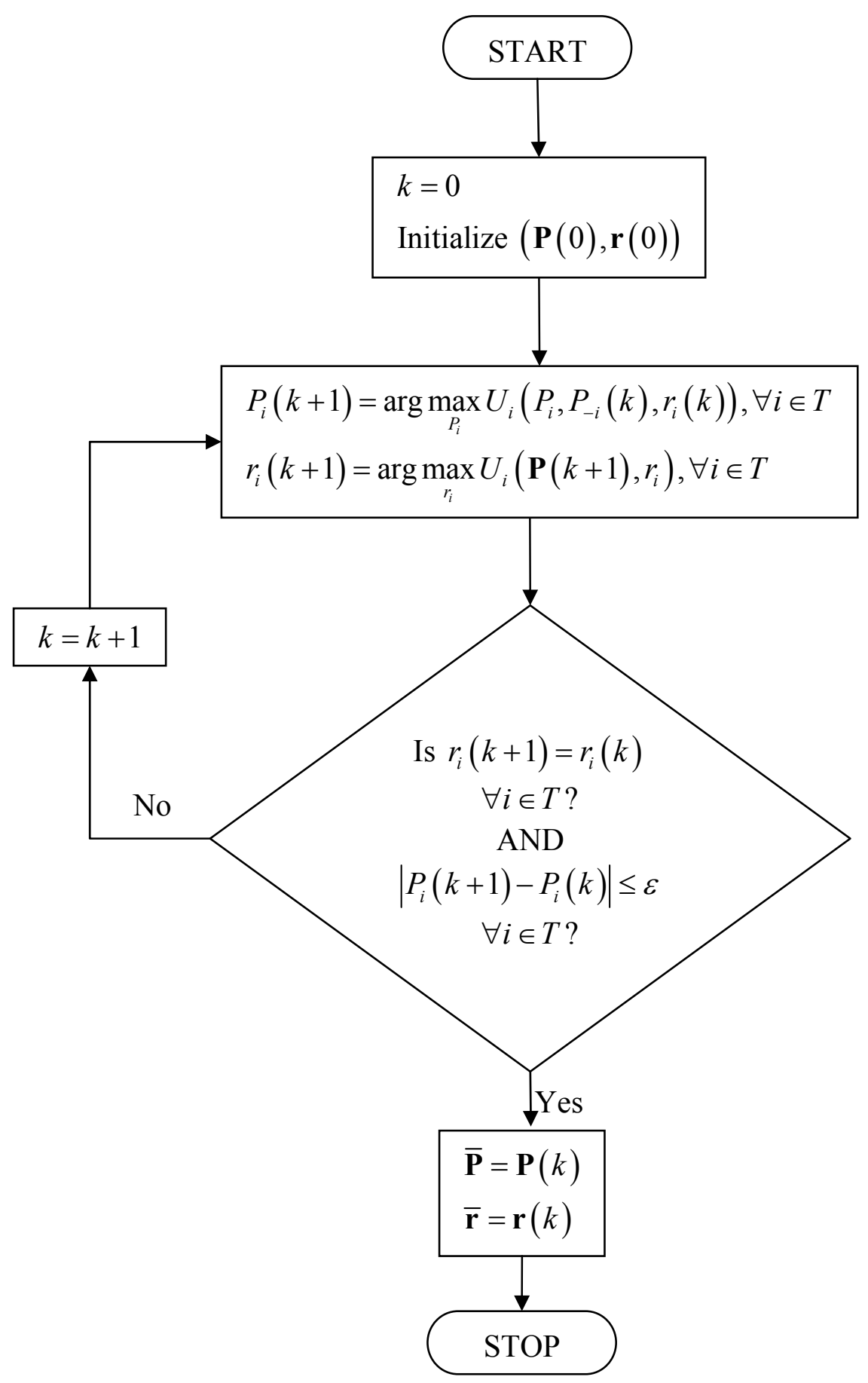

Figure 4-7 Algorithm LAG 


\subsubsection{Convergence of Algorithm LAG}

An important property of a distributed, iterative algorithm is its convergence. From the preceding development of Algorithm LAG, it becomes apparent that proof of its convergence is equivalent to proof that it will always result in the discovery of a Nash Equilibrium. To elaborate, when Algorithm LAG converges, no player can profitably deviate from its present action. All deviations would lead to a reduction in utility. By Definition 3-11, this must be a NE.

To demonstrate convergence of Algorithm LAG, we follow the approach outlined in Chapter 7 of [23]. In this approach, an algorithm is regarded as being a point-to-set map, say $\mathbf{A}$, that assigns a subset of the domain $X$ to each point $\mathbf{x}_{k} \in X$. An iteration of $\mathbf{A}$ is represented by the equation $\mathbf{x}_{k+1} \in \mathbf{A}\left(\mathbf{x}_{k}\right)$. We define the algorithmic map $\mathbf{M}$ for Algorithm LAG in Definition 4-2.

\section{Definition 4-2 Algorithmic Map M}

In our work, we define the algorithmic map $\mathbf{M}: A \rightarrow A$ to describe Algorithm LAG (Figure 4-7) as a sequence consisting of the following two interchangeable steps.

1. At iteration $k$, for each $i \in T$,

$$
\begin{aligned}
r_{i}(k+1) & =r^{l}, \text { iff. } U_{i}\left(\mathbf{P}(k), r^{l}\right)>U_{i}\left(\mathbf{P}(k), r^{j}\right), \forall r^{j} \in R-\left\{r^{l}\right\} \\
& =\min \left(r^{j}, r^{l}\right), \text { if } U_{i}\left(\mathbf{P}(k), r^{l}\right)=U_{i}\left(\mathbf{P}(k), r^{j}\right)
\end{aligned}
$$

2. At iteration $k$, for each $i \in T$,

$$
P_{i}(k+1)=\underset{P_{i} \in P}{\arg \max } U_{i}\left(P_{i}, P_{-i}(k), r_{i}(k+1)\right)
$$

From Proposition 4-1, $U_{i}$ has a single global maximum in its argument $P_{i}$ as long as $r_{i}$ is fixed, which, in this step, is indeed the case. This ensures that the left-hand side of Equation (4-21) is single-valued.

Since the penalty function $C_{i}$ is not a function of $r_{i}$, it is easy to show that step 1 of Definition 4-2 results in selection of ALP $r^{j}$ if and only if $L\left(\gamma, r^{j}\right)>L\left(\gamma, r^{l}\right), r^{l} \neq r^{j}$. If, for a particular $r^{l}, L\left(\gamma, r^{j}\right)=L\left(\gamma, r^{l}\right), r^{l} \neq r^{j}$, the lower of the two ALP values is chosen. Note that this choice is arbitrary. A mapping from SINR to $r$ is possible, based on the ranges of SINR where one value of the parameter $r$ produces greater throughput than the other. This is illustrated in Figure 4-8 for the GPRS example described in Section 4.2.5. 


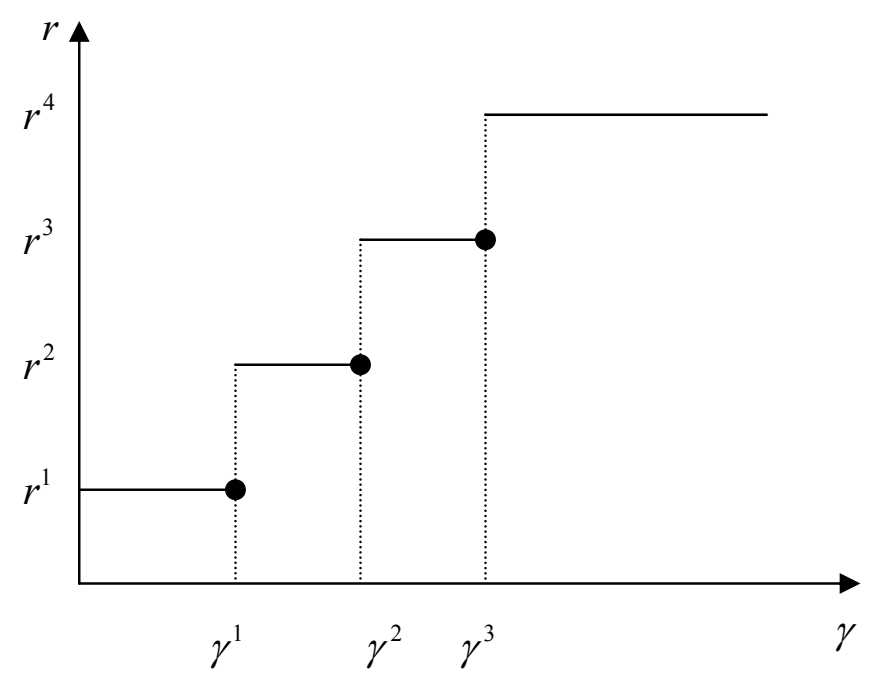

Figure 4-8 Mapping from $\gamma$ to $r$ for the GPRS example

In Figure 4-8, the points $\gamma^{1}, \gamma^{2}$, and $\gamma^{3}$ are defined as follows. $\gamma_{i}=\gamma^{j}, j \in\{1,2,3\}$, when $L_{i}\left(\mathbf{P}, r^{j}\right)=L_{i}\left(\mathbf{P}, r^{j+1}\right)$. At these points, by Equation (4-20), $r_{i}=r^{j}$ since the lower of the two values of $r_{i}$ is selected. The labeling of discontinuities in Figure 4-8 illustrates this. This relationship can also be equivalently defined in more general terms, as a mapping from $\mathbb{R} \rightarrow R$, as shown in Equation (4-22).

$$
r_{i}(k+1)=r^{n}, \quad \gamma^{n-1}<\gamma_{i} \leq \gamma^{n}
$$

where

$$
\begin{aligned}
& (i) \gamma^{0}=-\infty \\
& (\text { ii }) \gamma^{|\mathrm{R}|}=\infty \\
& (\text { iii }) \gamma^{n}=\left\{\gamma_{i}: L_{i}\left(\mathbf{P}, r^{n}\right)=L_{i}\left(\mathbf{P}, r^{n+1}\right)\right\} \\
& \forall n \in\{1,2, \ldots,|R|\}
\end{aligned}
$$

$\gamma_{i}(\mathrm{~dB})$ is defined in Equation (4-6).

Step 2 of Definition 4-2 assumes that $r_{i}$ is fixed during the course of the computation. Recalling Proposition 4-1, this step will discover the unique global maximum. In subsequent proofs, we make the following important assumption (Assumption 4-1) regarding the computation of Step 2 of Definition 4-2. A plethora of non-linear programming techniques are available to solve this sub-problem. Detailed descriptions of these techniques are available in [23]. In all examples presented in this thesis, we have allowed our simulation package $\mathrm{MATLAB}^{\mathrm{TM}}$ to select an appropriate approach. In all cases, a Successive Quadratic Programming algorithm with Quasi-Newton approximations produced satisfactory results for a seven-player game. 


\section{Assumption 4-1}

The numerical algorithm used to solve the non-linear program:

$$
\text { Find } P_{i} \text { to maximize } U_{i}\left(\mathbf{P}, r_{i}\right) \text { subject to } P_{i} \in P \text {, }
$$

always converges to a solution in $A$.

We now describe Algorithm LAG in Figure 4-7, utilizing the map $\mathbf{M}$ described in Definition 4-2.

\section{Definition 4-3 Algorithm LAG}

1. Initialization: Let $\mathbf{a}_{1} \in A$ be the starting point of Algorithm LAG. Set iteration count $k=1$.

2. Iterate: $\mathbf{a}_{k+1}=\mathbf{M}\left(\mathbf{a}_{k}\right)$.

3. Stop, if $\mathbf{a}_{k+1} \in \Omega(k)$, where $\Omega(k)$ is the solution set at iteration $k$. The solution set at any iteration $k$ is defined in Equation (4-23).

$$
\Omega(k)=\left\{(\mathbf{P}, \mathbf{r}):\left|P_{i}-P_{i}(k)\right| \leq \varepsilon, \varepsilon>0, \forall i, \text { and } \mathbf{r}=\mathbf{r}(k)\right\}
$$

4. Else, set $k=k+1$ and return to step 2 .

We now turn our attention to the convergence analysis of Algorithm LAG. In the following Lemma, we prove that the utility function $U_{i}$ is continuous over $A_{i}$. This result will be used in subsequent proofs.

\section{Lemma 4-5}

The utility function $U_{i}$ is continuous over $A_{i}$.

Proof:

Continuity is defined in Definition 3-8. Consider any two arbitrarily selected points $A_{i}^{0}=\left(P_{i}, r_{i}\right)$ and $A_{i}^{1}=\left(P_{i}^{\prime}, r_{i}^{\prime}\right)$, in $A_{i}$. Since both $P$ and $R$ are bounded, it is possible to find $\delta>0$, such that $\left|A_{i}^{1}-A_{i}^{0}\right|<\delta$. It is easy to see that $0 \leq L_{i} \leq \max _{r_{i} \in R} \alpha\left(r_{i}\right)$ and $-K P_{\max }^{q} \leq-C_{i} \leq 0$. Hence, $-K P_{\max }^{q} \leq U_{i} \leq \max _{r_{i} \in R} \alpha\left(r_{i}\right)$. Then, given $\varepsilon>0$, we must have $\left|U_{i}\left(A_{i}^{1}\right)-U_{i}\left(A_{i}^{0}\right)\right|<\varepsilon$. Since $U_{i}$ is bounded, it is possible to make $\varepsilon$ as large as necessary so that, for any $\delta>0,\left|A_{i}^{1}-A_{i}^{0}\right|<\delta$. Alternatively, lets suppose we shrink $\varepsilon$, such that $\varepsilon \rightarrow 0$. Then, as we correspondingly shrink $\delta$, we must have $r_{i}^{\prime}=r_{i}$, since the set of ALP $R$ is discrete, and the $\delta$-neighborhood of any $r_{i}$ contains only a single point, i.e. $r_{i}$ 
itself. Hence, $\left|U_{i}\left(A_{i}^{1}\right)-U_{i}\left(A_{i}^{0}\right)\right|=\left|\Delta U_{i}\right| \approx\left|\frac{\partial U_{i}}{\partial P_{i}}\right| \Delta P_{i}$. Since we now have, $\Delta U_{i}=\varepsilon, \Delta P_{i}=\delta$ and $\frac{\partial U_{i}}{\partial P_{i}}$ is continuous for any $A_{i}^{0}=\left(P_{i}, r_{i}\right)$ (as shown in Lemma 4-3), choosing $\delta \leq \frac{\varepsilon}{\left|\frac{\partial U_{i}\left(A_{i}^{0}\right)}{\partial P_{i}}\right|}$ results in $\left|U_{i}\left(A_{i}^{1}\right)-U_{i}\left(A_{i}^{0}\right)\right|<\varepsilon$. Hence, the proof is complete.

The idea of a closed map is central to proving the convergence of algorithms using the algorithmic map approach. This concept is often one requirement amongst others in proving the convergence of algorithms. We present the definition of a closed map given in [23].

\section{Definition 4-4 Closed Map [23]}

Let $X$ and $Y$ be non-empty closed sets in Euclidean spaces. Let $\mathbf{A}: X \rightarrow Y$ be a point-toset map. The map A is said to be closed at $\mathbf{x} \in X$ if

$$
\begin{array}{ll}
\mathbf{x}_{k} \in X, & \mathbf{x}_{k} \rightarrow \mathbf{x} \\
\mathbf{y}_{k} \in \mathbf{A}\left(\mathbf{x}_{k}\right), & \mathbf{y}_{k} \rightarrow \mathbf{y}
\end{array}
$$

imply that $\mathbf{y} \in \mathbf{A}(\mathbf{x})$. The map $\mathbf{A}$ is said to be closed on $Z \subseteq X$ if it is closed at each point in $\mathrm{Z}$.

We now show that the algorithmic map $\mathbf{M}$ is not closed. We shall eventually demonstrate that this property is not strong enough to preclude convergence of Algorithm LAG.

\section{Proposition 4-4}

The algorithmic map $\mathbf{M}$ is not closed.

Proof:

To investigate whether the map $\mathbf{M}$ is closed, we directly apply Definition 4-4. It is sufficient to find a single point at which $\mathbf{M}$ is not closed to prove this assertion. We first define a converging sequence $\{\mathbf{X}(k)=(\mathbf{P}(k), \mathbf{r}(k))\}$ in $A$. Let the limiting point of this sequence be denoted by the vector $\mathbf{X}=\left(\mathbf{P}_{x}, \mathbf{r}_{x}\right)$. Since the set of ALP is discrete, a converging sequence of ALPs implies that there exists $K$ such that, for all $k \geq K, \mathbf{r}(k)=\mathbf{r}(K)=\mathbf{r}_{x}$.

The image produced by mapping $\{\mathbf{X}(k)\}$ under $\mathbf{M}$ is the sequence $\left\{\mathbf{Y}(k)=\mathbf{M}(\mathbf{X}(k))=\left(\mathbf{P}^{*}(k), \mathbf{r}^{*}(k)\right)\right\}$. Does this sequence converge? The sequence 
$\{\mathbf{X}(k)=(\mathbf{P}(k), \mathbf{r}(k))\}$ produces a sequence of powers that converge to $\mathbf{P}_{x}$ and ALPs that converge to $\mathbf{r}_{x}$. Since the SINR $\gamma$ is continuous over $A,\{\mathbf{X}(k)\}$ generates a converging sequence of SINR vectors $\{\gamma(k)\} \rightarrow \gamma_{x}$. We are free to select $\{\mathbf{X}(k)\}$ in an arbitrary manner. Suppose, we specifically select $\{\mathbf{X}(k)\}$ such that, $\gamma_{x, 1}=\gamma^{1}$ and $\left\{\gamma_{1}(k)\right\}$ approaches $\gamma^{1}$ from the right. It is easy to visualize this with the help of Figure 4-8.

From Lemma 4-5, since all $U_{i}$ are continuous over $A_{i},\{\mathbf{X}(k)\}$ will generate a converging sequence of utility function profiles.

$$
\left\{U_{i}\left(\mathbf{P}(k), r_{i}(k)\right)\right\} \rightarrow U_{i}\left(\mathbf{P}_{x}, r_{i, x}\right), \forall i
$$

Then, from Equation (4-22), there must exist $K^{\prime}$, such that for $k \geq K^{\prime}$,

$$
r_{i}^{*}(k+1)=r_{i}^{*}(k)=r_{i}^{*}\left(K^{\prime}\right), \forall i
$$

In this particular instance, using Equation (4-22), we have $r_{y, 1}=r^{2}$, since, recalling our definition of $\{\mathbf{X}(k)\},\left\{\gamma_{1}(k)\right\} \rightarrow \gamma^{1}$, but $\gamma_{1}(k)>\gamma^{1}$. Let the convergence point for the ALP be denoted by $\mathbf{r}_{y}$.

If the utility function profiles and ALPs converge as shown in Equation (4-24) and Equation (4-25) respectively, and Assumption 4-1 holds, it follows that step 2 of $\mathbf{M}$ in Definition 4-2 will yield a converging sequence of powers $\left\{P_{i}^{*}(k)\right\} \rightarrow \underset{P_{i} \in P}{\arg \max } U_{i}\left(P_{i}, P_{-i, x}, r_{i, x}\right)$, whose accumulation point is denoted by $\mathbf{P}_{y}$. Therefore, the sequence $\{\mathbf{Y}(k)\}$ converges to a point $\mathbf{Y}=\left(\mathbf{P}_{y}, \mathbf{r}_{y}\right)$ such that $r_{y, 1}=r^{2}$, as demonstrated earlier.

According to Definition 4-4, to prove that $\mathbf{M}$ is closed it needs to be shown that $\mathbf{Y}^{*}=\mathbf{Y}$, where $\mathbf{Y}^{*}=\mathbf{M}(\mathbf{X})$. Since $\mathbf{P}_{x}$ maps to $\gamma_{x}$, we have $\gamma_{1}^{*}=\gamma_{x, 1}=\gamma^{1}$. From the careful application of Equation (4-22), it follows that $r_{1}^{*}=r^{1} \neq r_{y, 1}$. We have, therefore, found a point $\mathbf{X}$ at which the mapping $\mathbf{M}$ is not closed. Therefore, by Definition 4-4, $\mathbf{M}$ is not closed over the set $A$ and the proof is complete.

It is possible for an algorithm with a map that is not closed to converge to a point in its solution set. If it is possible to decompose the map of an algorithm into two maps, one of which is closed, and the other possibly not, then, provided certain other conditions are met, the algorithm may still converge. The convergence theorem for algorithms with composite maps from [23] is stated here in Theorem 4-1, in a slightly modified form. The condition in the theorem below, which requires that the sequence produced by the algorithm be contained in a compact set, is from Polak [74] and replaces a more stringent condition in [23]. However, the proof of the theorem remains unchanged after this substitution. 


\section{Theorem 4-1 Convergence of Algorithms with Composite Maps}

Let $X$ be a non-empty closed set in $E_{n}$, and let $\Omega \subseteq X$ be a non-empty solution set. Let $\beta: E_{n} \rightarrow E_{1}$ be a continuous function, and consider the point-to-set map $\mathbf{C}: X \rightarrow X$ satisfying the following property: Given $\mathbf{x} \in X$, then $\beta(\mathbf{y}) \leq \beta(\mathbf{x})$ for $\mathbf{y} \in \mathbf{C}(\mathbf{x})$. Let $\mathbf{B}: X \rightarrow X$ be a point-to-set map that is closed over the complement of $\Omega$, and satisfies $\beta(\mathbf{y})<\beta(\mathbf{x})$ for each $\mathbf{y} \in \mathbf{B}(\mathbf{x})$, if $\mathbf{x} \notin \Omega$. Now, consider the algorithm defined by the composite map $\mathbf{A}=\mathbf{C B}$. Given $\mathbf{x}_{1} \in X$, suppose that the sequence $\left\{\mathbf{x}_{k}\right\}$ is generated as follows:

$$
\text { If } \mathbf{x}_{k} \in \Omega \text {, stop; otherwise, let } \mathbf{x}_{k+1} \in A\left(\mathbf{x}_{k}\right) \text {, replace } k \text { by } k+1 \text {, and repeat. }
$$

Suppose the sequence $\left\{\mathbf{x}_{k}\right\}$ is contained in a compact subset $\Lambda$ of $X$. Then, either the algorithm stops in a finite number of steps with a point in $\Omega$, or all accumulation points of $\left\{\mathbf{x}_{k}\right\}$ belong to $\Omega$.

It is quite straightforward to view the algorithmic map $\mathbf{M}$ as being composed of two maps: $\mathbf{C}$, consisting of step 1 of $\mathbf{M}$, and $\mathbf{B}$, consisting of step 2 of $\mathbf{M}$ (see Definition 4-2). The maps $\mathbf{B}$ and $\mathbf{C}$ are defined in Definition 4-5 and Definition 4-6, respectively. The composite map can be expressed as $\mathbf{M}=\mathbf{C B}$. This composite mapping involves a mapping under $\mathbf{B}$, followed by a mapping under $\mathbf{C}$.

\section{Definition 4-5 Algorithmic Map B}

$\mathbf{B}: A \rightarrow A$

Let $(\mathbf{P}, \mathbf{r})$ be the input to $\mathbf{B}$ and $\left(\mathbf{P}^{\prime}, \mathbf{r}^{\prime}\right)$ be its output. Then, for each $i \in T$

$$
r_{i}^{\prime}=r_{i}
$$

$P_{i}^{\prime}=\underset{P_{i} \in P}{\arg \max } U_{i}\left(P_{i}, P_{-i}, r_{i}\right)$

From Proposition 4-1, $U_{i}$ has a single global maximum in its argument $P_{i}$ since $r_{i}$ is fixed. This ensures that $P_{i}^{\prime}$ is single-valued.

\section{Definition 4-6 Algorithmic Map C}

$\mathrm{C}: A \rightarrow A$

Let $(\mathbf{P}, \mathbf{r})$ be the input to $\mathbf{C}$ and $\left(\mathbf{P}^{\prime}, \mathbf{r}^{\prime}\right)$ be its output. Then, for each $i \in T$ $P_{i}^{\prime}=P_{i}$ 
$r_{i}^{\prime}=r^{n}, \quad \gamma^{n-1}<\gamma_{i} \leq \gamma^{n}$

where

(i) $\gamma^{0}=-\infty$

(ii) $\gamma^{|\mathrm{R}|}=\infty$

(iii) $\gamma^{n}=\left\{\gamma_{i}: L_{i}\left(\mathbf{P}, r^{n}\right)=L_{i}\left(\mathbf{P}, r^{n+1}\right)\right\}$

$\forall n \in\{1,2, \ldots,|R|\}$

$\gamma_{i}(\mathrm{~dB})$ is defined in Equation (4-6).

As an intermediate step in proving the convergence of Algorithm LAG, we show in Proposition 4-5 that the algorithmic map $\mathbf{B}$ is closed. The map $\mathbf{C}$ is probably not closed, but Theorem 4-1 does not necessitate that both maps be closed.

\section{Proposition 4-5}

The algorithmic map B is closed.

Proof:

Applying Definition 4-4 to investigate whether the map B is closed, we first define an arbitrary converging sequence $\{\mathbf{X}(k)=(\mathbf{P}(k), \mathbf{r}(k))\}$ in $A$. Let the limiting point of this sequence be denoted by the vector $\mathbf{X}=\left(\mathbf{P}_{x}, \mathbf{r}_{x}\right)$. Since the set of ALPs is discrete, a converging sequence of ALPs, implies that there exists $K$, such that, for all $k \geq K$,

$$
\mathbf{r}(k)=\mathbf{r}(K)=\mathbf{r}_{x}
$$

The image produced by mapping $\{\mathbf{X}(k)\}$ under $\mathbf{B}$ is the sequence $\left\{\mathbf{Y}(k)=\mathbf{B}(\mathbf{X}(k))=\left(\mathbf{P}^{*}(k), \mathbf{r}^{*}(k)\right)\right\}$. Does this sequence converge? The sequence $\{\mathbf{X}(k)=(\mathbf{P}(k), \mathbf{r}(k))\}$ produces a sequence of powers that converge to $\mathbf{P}_{x}$ and ALP vectors that converge to $\mathbf{r}_{x}$. By the definition of $\mathbf{B}$ in Definition 4-5, the ALPs remain unchanged. Hence, we must have $r_{i}^{*}(k)=r_{i}(k)$. Using Equation (4-26), clearly $\left\{\mathbf{r}^{*}(k)\right\} \rightarrow \mathbf{r}_{x}$. Let the convergence point for the ALP vectors, produced by mapping $\{\mathbf{X}(k)\}$ under $\mathbf{B}$, be denoted by $\mathbf{r}_{y}$. It follows that $\mathbf{r}_{y}=\mathbf{r}_{x}$.

From Lemma 4-5, since $U_{i}$ is continuous over $A_{i},\{\mathbf{X}(k)\}$ will generate a converging sequence of utility function profiles.

$$
\left\{U_{i}\left(\mathbf{P}(k), r_{i}(k)\right)\right\} \rightarrow U_{i}\left(\mathbf{P}_{x}, r_{i, x}\right), \forall i
$$

Thus, we must have, 


$$
\left\{\max _{P_{i} \in P} U_{i}\left(P_{i}, P_{-i}(k), r_{i}(k)\right)\right\} \rightarrow \max _{P_{i} \in P} U_{i}\left(P_{i}, P_{-i, x}, r_{i, x}\right)
$$

If Assumption 4-1 holds, we conclude that map B yields a converging sequence of powers $\left\{P_{i}^{*}(k)\right\} \rightarrow \underset{P_{i} \in P}{\arg \max } U_{i}\left(P_{i}, P_{-i, x}, r_{i, x}\right)$ with accumulation point denoted by $\mathbf{P}_{y}$.

Therefore, $\{\mathbf{Y}(k)\}$ converges to a point $\mathbf{Y}=\left(\mathbf{P}_{y}, \mathbf{r}_{x}\right)$, since $\mathbf{r}_{y}=\mathbf{r}_{x}$. Further, let $\mathbf{Y}^{\prime}=\mathbf{B}(\mathbf{X})$. Then, $P_{i}^{\prime}=\underset{P_{i} \in P}{\arg \max } U_{i}\left(P_{i}, P_{-i, x}, r_{i, x}\right)$ and $r_{i}^{\prime}=r_{i, x}$, by definition of map $\mathbf{B}$

(Definition 4-5). Hence, $\mathbf{Y}^{\prime}=\mathbf{Y}$. Thus, by Definition 4-4, the map $\mathbf{B}$ is closed and the proof is complete.

We are now equipped with the results we need to prove that Algorithm LAG (Definition 4-3) does indeed converge.

\section{Proposition 4-6}

The Algorithm LAG converges to a Nash Equilibrium, provided Assumption 4-1 holds.

Proof:

The proof follows from a systematic application of Theorem 4-1. Note that the definition $\mathbf{M}=\mathbf{C B}$ is in an order that permits direct application of this theorem. We first define the descent function $\beta$, at iteration $k$, as follows.

$$
\beta(\mathbf{x}, k)=-\sum_{i=1}^{N} U_{i}\left(x_{i}, I_{i}(k)\right), \quad \mathbf{x} \in A, \text { and } x_{i}=\left(P_{i}, r_{i}\right)
$$

Here, $I_{i}(k)=\sum_{j \neq i}^{N} G_{i, j} P_{j}(k)+n_{i}$ is the interference at $i$ at iteration $k$.

1. $\beta$ is the sum of $N$ continuous functions (using Lemma 4-5). Hence, $\beta$ is continuous.

2. We first show that if $\mathbf{y}=C(\mathbf{x}), \mathbf{x} \in A$, then $\beta(\mathbf{y}, k) \leq \beta(\mathbf{x}, k)$, at iteration $k$. Let $\mathbf{x}=\left(\mathbf{P}_{x}, \mathbf{r}_{x}\right)$ and $\mathbf{y}=\left(\mathbf{P}_{y}, \mathbf{r}_{y}\right)$. By definition of $\mathbf{C}$ (Definition 4-6), $\mathbf{P}_{y}=\mathbf{P}_{x}$. If $\mathbf{r}_{y}=\mathbf{r}_{x}$, as would occur when the ALPs do converge, then, from Equation (4-29), we must have $\beta(\mathbf{y}, k)=\beta(\mathbf{x}, k)$. It is important to realize that this does not necessarily mean that the algorithm terminates here, since the powers might not have converged. If the ALPs have not converged either, $\exists i$ such that $U_{i}\left(\mathbf{P}_{y}=\mathbf{P}_{x}, r_{i, y}\right)>U_{i}\left(\mathbf{P}_{x}, r_{i, x}\right)$. Thus, in this case, $\beta(\mathbf{y}, k)<\beta(\mathbf{x}, k)$. Note that it is not possible for $\beta(\mathbf{y}, k)>\beta(\mathbf{x}, k)$ since this would be inconsistent with the definition of the map $\mathbf{M}$ and becomes apparent by studying Equation (4-20).

3. The map B is closed, by Proposition 4-5.

4. We now show that, given $\mathbf{y}=\mathbf{B}(\mathbf{x}), \mathbf{x} \in A$, then $\beta(\mathbf{y}, k)<\beta(\mathbf{x}, k)$, if $\mathbf{x} \notin \Omega(k-1)$, where $\Omega(k-1)$ is the solution set at iteration $k$-1, as defined in Equation (4-23). 
By contradiction, suppose that at iteration $k, \beta(\mathbf{y}, k)=\beta(\mathbf{x}, k)$ and the algorithm has not converged, i.e. $\mathbf{y} \notin \Omega(k)$, where $\mathbf{x}=\left(\mathbf{P}_{x}, \mathbf{r}_{x}\right)$ and $\mathbf{y}=\mathbf{B}(\mathbf{x})=\left(\mathbf{P}_{y}, \mathbf{r}_{y}\right)$. Assumption 4-1 implies that $\mathbf{y}$ exists. Since by definition of the map B (Definition 4-5), $\mathbf{r}_{y}=\mathbf{r}_{x}$. Then, we must have $U_{i}\left(P_{i, y}, P_{-i, x}, r_{i, x}\right)=U_{i}\left(P_{i, x}, P_{-i, x}, r_{i, x}\right) \forall i$. Note that this is the only possibility, since Equation (4-21) precludes the occurrence of the only other possibility $U_{i}\left(P_{i, y}, P_{-i, x}, r_{i, x}\right)<U_{i}\left(P_{i, x}, P_{-i, x}, r_{i, x}\right)$, for any $i$. This implies that $P_{i, y}=P_{i, x}$.

Obviously, we must have $\left|P_{i, y}-P_{i, x}\right|<\varepsilon$, implying that the algorithm $\mathbf{M}$ has in fact converged, i.e. $\mathbf{y} \in \Omega(k)$. Note that, since $P_{i, x}=P_{i}(k)$ and $P_{i, y}=P_{i}(k+1)$, at the next iteration $k+1$, the set of $U_{i}$ will be identical to what it was at iteration $k$. Thus, when the powers have converged, the ALPs cannot change. The convergence implies that our original assumption, $\beta(\mathbf{y}, k)=\beta(\mathbf{x}, k)$, must be false. Hence, we must strictly have $\beta(\mathbf{y}, k)<\beta(\mathbf{x}, k)$. Note that it is not possible for $\beta(\mathbf{y}, k)>\beta(\mathbf{x}, k)$ since this would be inconsistent with the definition of the map $\mathbf{M}$ and is evident from Equation (4-21).

5. Any sequence produced by the mapping $\mathbf{M}$ is contained in the set $A$. The set $A$ is compact by Lemma 4-1, since $A_{i}$ is compact, $\forall i \in T$.

Since all the conditions required by Theorem 4-1 are satisfied, the algorithm described by the map $\mathbf{M}$ converges. Suppose that the accumulation point is denoted by $\overline{\mathbf{a}}=(\overline{\mathbf{P}}, \overline{\mathbf{r}})$. Then, at the point of convergence, the following property must be satisfied for all $i \in T$.

$$
U_{i}\left(\bar{a}_{i}, \bar{a}_{-i}\right) \geq U_{i}\left(a_{i}, \bar{a}_{-i}\right), \forall a_{i} \in A_{i}
$$

Comparing Equation (4-30) with Equation (3-4), we observe that Equation (4-30) is simply the definition of a NE. Thus, we have shown that the convergence point of algorithm LAG is a NE and the proof is complete.

\subsection{Results and Discussion}

Having established a model for the LAG based on the principles of strategic-form games in Section 4.2, and conceived an algorithm that specifies a pattern of play leading to a NE in Section 4.4, we present in this section, several interesting results based on the GPRS example in Section 4.2.5. These results are also published in [72]. For example, it is not obvious yet what allocation of power and rate will result from the execution of Algorithm LAG. While this algorithm has been shown in Section 4.4 to converge without making any assumptions on its initial conditions, the sensitivity of the NE to different sets of initial conditions is yet to be investigated. We have not been able to make any general analytical predictions on this subject and, therefore, the results can be expected to provide valuable insight on the same. We also investigate the use of different penalty function 
parameters and their impact on power consumption and system throughput. The equally interesting matter of comparing these results with those obtained using systems techniques, for the same network configuration, is postponed until the next chapter.

\subsubsection{System Configuration}

The basis for our results is the seven-cell star type network configuration illustrated in Figure 4-9. A cutaway consisting of seven co-channel cells is depicted. The intervening area is occupied by adjacent channels, which are not considered in the game. It is important to note that only the first-tier of co-channel interferers relative to the central cell is considered in the subsequent discussion. The interference from the second and third tiers is usually not negligible, but this simplification helps in limiting the computational scale of the problem. Consequently, the ensuing results will be optimistic.

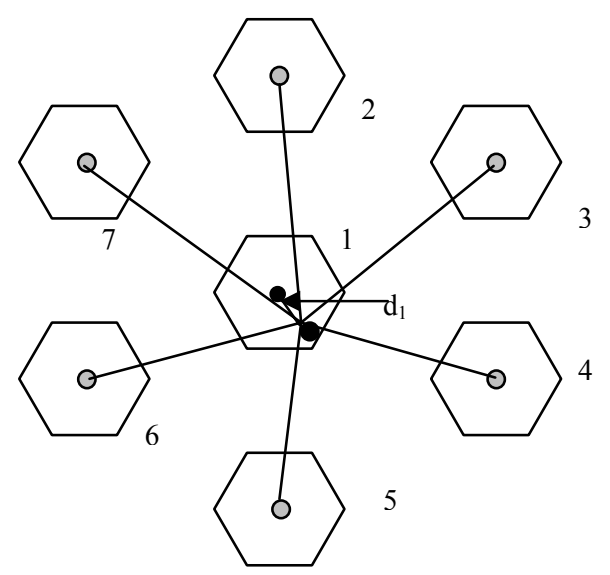

Figure 4-9 Seven-player game, re-use factor $=3$. A set of co-channel cells is shown.

The downlink case is considered in all the forthcoming examples. The base-stations of the cells numbered 1 to 7 are the co-channel interferers, and constitute the set $T$. In computing the path loss coefficients, the distance from each interferer to the receiver of interest is approximated as the distance between the centers of their cells. The frequency re-use factor is set to 3. This is the minimum possible value in a GSM system [5]. With this setting, the first-tier of interfering base stations lie on a circle of radius $3 \mathrm{R}$, where $\mathrm{R}$ is the cell radius. The path loss model is log-distance but variations due to log-normal shadowing have not been considered. This permits the establishment of a path loss matrix that remains identical between simulation trials. Noise is referenced to the maximum possible received power of the weakest user, as shown in Equation (4-31), and is identical for all $i \in T$.

$$
n_{i}=\frac{\min _{i \in T}\left(G_{i i}\right) P_{\max }}{\mathrm{SNR}} \quad \forall i \in T
$$

The maximum transmitter power per link $P_{\max }$ is $10 \mathrm{~mW}$, while minimum transmitter power $P_{\min }$ is $0 \mathrm{~mW}$. The positioning of the terminals in the system, which stays the same in all examples that follow, is shown in Table 4-3. 
Table 4-3 System Configuration

\begin{tabular}{|l|c|c|c|c|c|c|c|}
\hline Player $i$ & 1 & 2 & 3 & 4 & 5 & 6 & 7 \\
\hline$d_{i} / \mathrm{R}$ & 1 & 0.1 & 1 & 0.5 & 1 & 1 & 1 \\
\hline
\end{tabular}

In Table 4-3, the first row indexes the players in accordance with the numbering scheme given in Figure 4-9. The second row indicates the distance $d_{i}$ of a terminal $i \in T$ from its serving base station, normalized by the cell radius $R$. A value of 1 is the worst case situation, where the handset is located at the cell boundary. Thus, the handset in cell 2 is 10 times closer to its base station than that in cell 1 . Movement of terminals is inconsistent with our assumption that the link gain matrix does not change for the convergence period of Algorithm LAG (Section 4.4) and is therefore, not permitted.

\subsubsection{Figures of Merit}

To compare and evaluate our results in a consistent manner, we propose a set of three figures of merit (FOM). FOM1 is the ratio of the sum of link throughputs, referred to as the system throughput, to the sum of the fractions of peak power consumed by the links, each fraction being referred to as the power consumption coefficient (PCC) of its link. The mathematical expression for FOM1 is given in Equation (4-32).

$$
F O M 1=\frac{\sum_{i=1}^{N} L_{i}}{\sum_{i=1}^{N} P_{i} / P_{\max }}
$$

FOM2 is the difference of system throughput and scaled sum of the PCCs, where the scaling factor is the peak throughput. The mathematical expression for FOM2 is given in Equation (4-33).

$$
\text { FOM } 2=\sum_{i=1}^{N} L_{i}-\max _{r \in R}\{\alpha(r)\} \cdot \sum_{i=1}^{N} P_{i} / P_{\max }
$$

FOM3 is simply the system throughput, as shown in Equation (4-34).

$$
\text { FOM } 3=\sum_{i=1}^{N} L_{i}
$$

The proposed FOMs require that the sum of throughput be maximized. However, the first two add the condition that power consumption be minimized. In all three cases, a higher FOM is preferable.

\subsubsection{Simulation Results}

Since our primary interest lies in the interference-limited case, we set signal to noise ratio (SNR) to $100 \mathrm{~dB}$. We begin our presentation of results with an example based on the model described in Section 4.5.1, in which the penalty function parameters are set to $K$ $=1, q=0.7$, and all players initially choose CS-1. The simulation, as expected, discovers the NE and the results for this trial are shown in Table 4-4. The second row of this table expresses, as a percentage, the PCC of each link in NE; the third displays the final coding 
schemes that each link has converged to; and the next two rows display the SINR attained on each link and their corresponding equilibrium throughputs, respectively.

Table 4-4 Example simulation, with $K=1, q=0.7$

\begin{tabular}{|l|c|c|c|c|c|c|c|}
\hline \multicolumn{1}{|c|}{ Player } & 1 & 2 & 3 & 4 & 5 & 6 & 7 \\
\hline$\left(P / P_{\max }\right) \times 100$ & 49 & 7 & 80 & 40 & 72 & 73 & 69 \\
\hline CS & 1 & 4 & 3 & 3 & 2 & 2 & 2 \\
\hline SINR $(\mathrm{dB})$ & 5.8 & 28.9 & 11.9 & 15.7 & 10 & 9.7 & 10.5 \\
\hline$L_{i}(\mathrm{kbps})$ & 4.2 & 13.8 & 7.7 & 9.8 & 6.4 & 6.2 & 6.7 \\
\hline \multicolumn{8}{|c|}{ System Throughput $=54.8 \mathrm{kbps}$} \\
\hline
\end{tabular}

The results in Table 4-4 are intuitive. The following inferences can be drawn from these results.

- The equilibrium rates reflect the channel quality of the respective links. The links that receive favorable channel conditions select higher code rates (CS-3 or CS-4). There is a direct correspondence between the SINR operating point of each link and its NE CS selection. A practical implementation would use an estimate of SINR, which it maps to an appropriate coding scheme [75] based on the cross-over points of the throughput-SINR curves in Figure 1-2. Table 4-5 identifies the mapping for these curves.

Table 4-5 SINR to CS mapping in GPRS

\begin{tabular}{|c|c|}
\hline SINR Region $(\mathrm{dB})$ & Coding Scheme \\
\hline $0-7$ & 1 \\
\hline $7-11$ & 2 \\
\hline $11-21$ & 3 \\
\hline $21-$ & 4 \\
\hline
\end{tabular}

- Link 1, which receives maximum interference by virtue of being at the center of the star configuration, cannot select a rate higher than CS-1.

- It is interesting to note, that despite the fact that the MS of link 3 resides at its cell boundary, link 3 selects CS-3, while similarly disposed links 5, 6 and 7, end up selecting CS-2. This can be explained by the fact that the sources of interference for link 3 include links 2 and 4, which receive favorable channel conditions and, hence, can maintain high throughput while operating at relatively lower power. This results in less interference for link 3, compared to the interference experienced by links 5, 6 and 7 , thereby allowing link 3 to operate in the higher SINR region corresponding to CS-3.

One can immediately notice from Table 4-4 that the PCCs of some terminals are rather high, implying that they are operating inefficiently. One suspects that this might be on account of too lenient pricing. The present results also motivate one to consider the 
impact of different initial CS choices on the NE. We address these topics in the following subsections.

\subsubsection{Sensitivity to Initial Conditions}

In Figure 4-10, the histogram of the equilibrium FOMs, resulting from several plays of $\mathrm{LAG}$, each of which is initialized with one of the $4^{7}$ different starting rates, is plotted for $K=1, q=2$ and an SNR of $100 \mathrm{~dB}$. In Figure 4-10, the NE CS vector is printed beside the histogram bar corresponding to that NE. The key observations from this exercise are as follows.

- There are just four well-defined, though closely spaced, NE.

- One of these equilibria clearly occurs far more frequently than the rest. This is the CS combination [ $\left[\begin{array}{lllllll}1 & 4 & 2 & 3 & 2 & 2 & 2\end{array}\right]$. In over 80 per cent of all cases, Algorithm LAG converges to this equilibrium.

- Observing the third pane of Figure 4-10, the variation in system throughput obtained at these equilibria is insignificant, the maximum being approximately $200 \mathrm{bps}$.
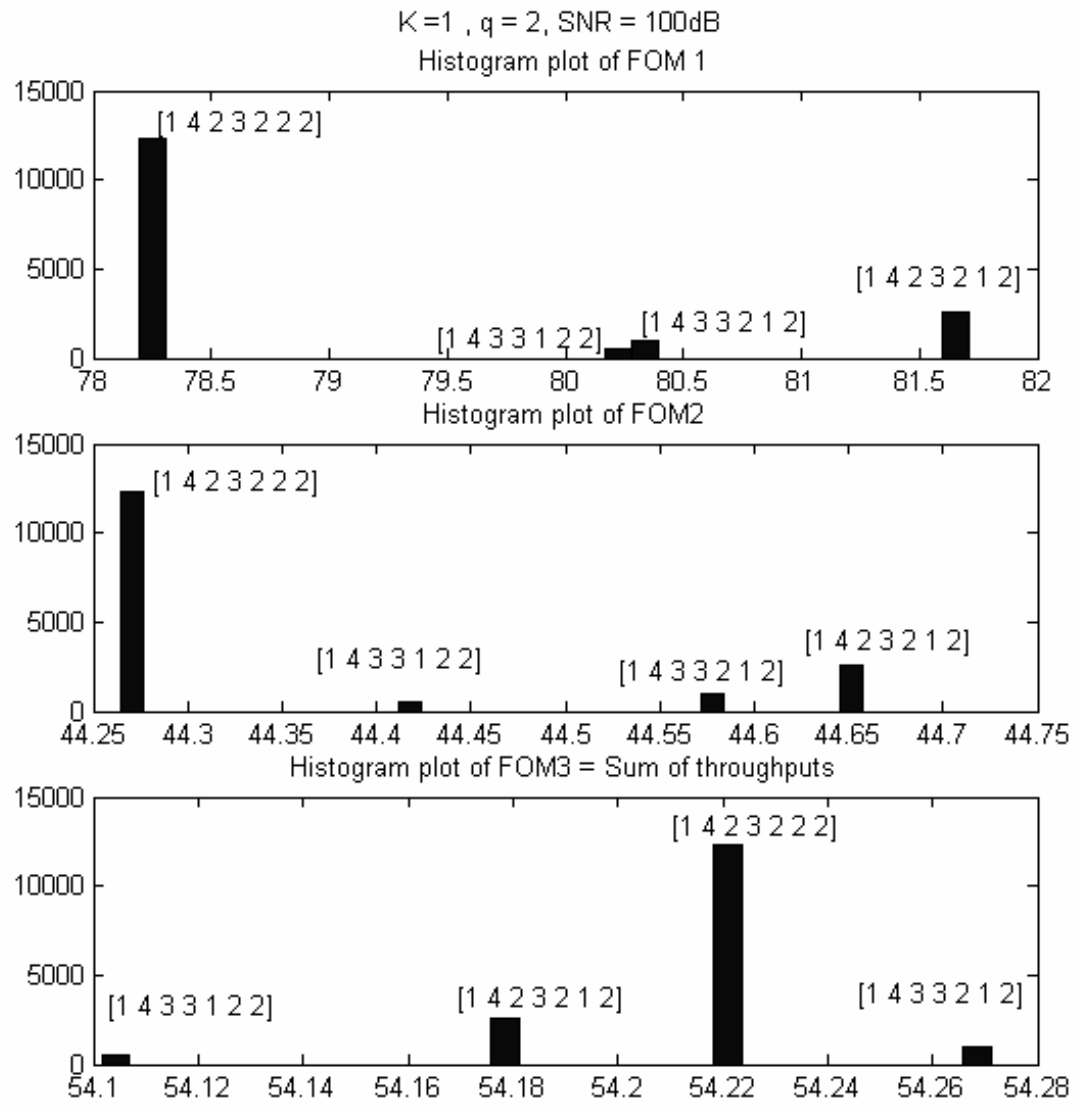

Figure 4-10 Histogram plot of FOMs in NE 


\subsubsection{Impact of Penalty Function Parameters ${ }^{7}$}

Recall that the penalty function parameters are $q$ and $K$. We maintain $K=1$ at all times and investigate the impact of $q$ on system performance. Figure 4-11 and Figure 4-13 illustrate the effect that $q$ has on the FOMs and power consumption, respectively. The former plots all 3 FOMs on the same axis versus $q$ whereas the latter plots the sum of the PCCs versus $q$. In Figure 4-12, a close-up of the variation in system throughput (FOM3), is illustrated. Due to scale, this was not clearly visible in Figure 4-11. We notice a clear peak in system throughput at $q \approx 0.7$. At this value, it can be observed from Figure 4-13, that the power consumption is nearly $60 \%$ of its maximum. There seems to be a convergence of system throughput, as $q$ increases, towards a value of approximately 53 kbps. However, FOM1 and FOM2 are small for lower values of $q$ due to their excessive penalizing of power consumption. In fact, Figure 4-13 suggests that selecting $q>1.5$ will be generally conservative of power. In Figure 4-11, FOM1 is observed to be maximized for $q=4.9$ and FOM2, for $q=3.6$. These FOMs, therefore, lead to a more powerconservative tuning of $q$. Such settings might be more germane when applying this model to an uplink situation where battery life is a critical design parameter.

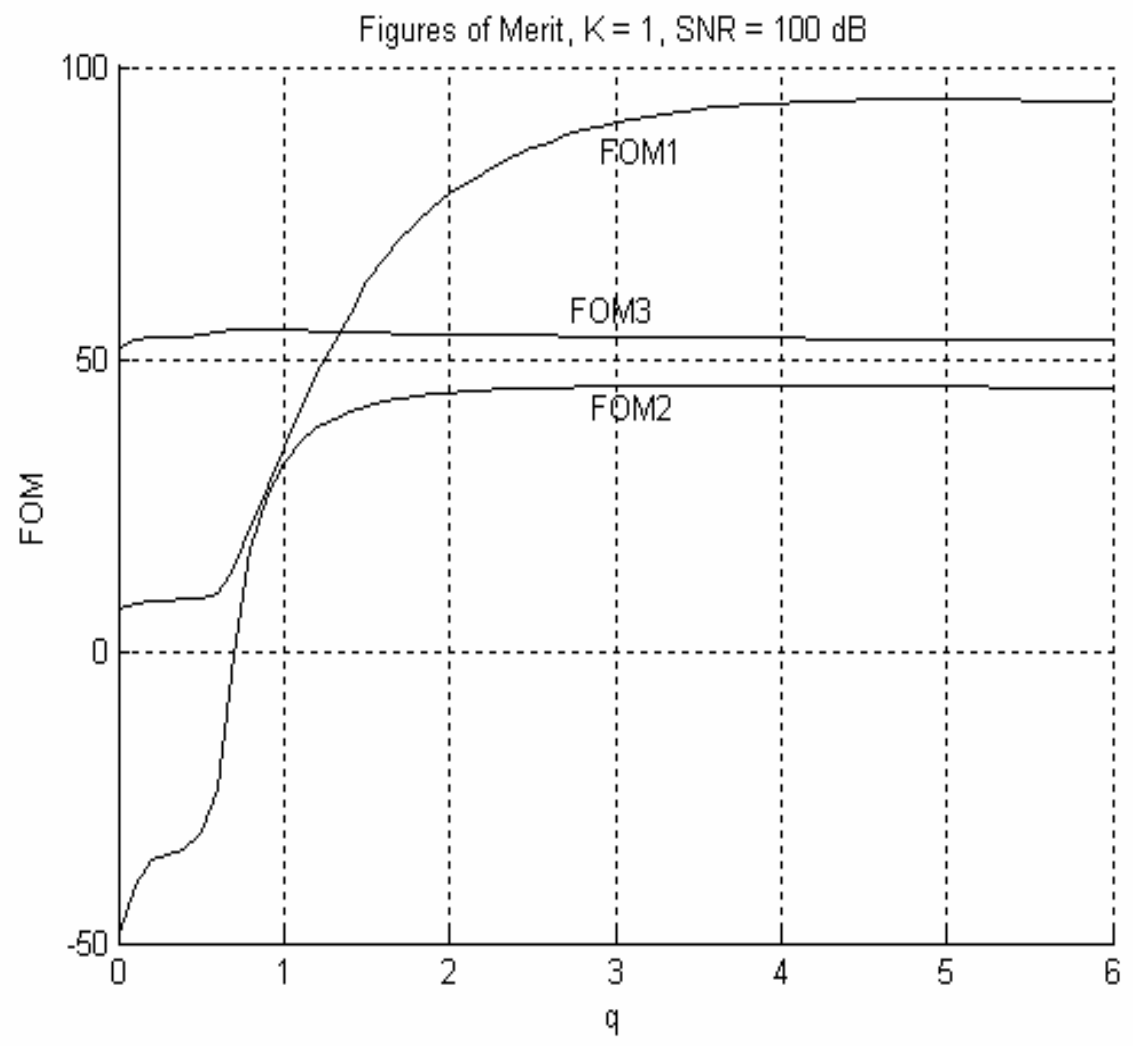

Figure 4-11 Effect of $q$ on FOMs, SNR $=100 \mathrm{~dB}$

\footnotetext{
${ }^{7}$ A lower power bound of $P_{\min }=0.1$ was used in these simulations.
} 


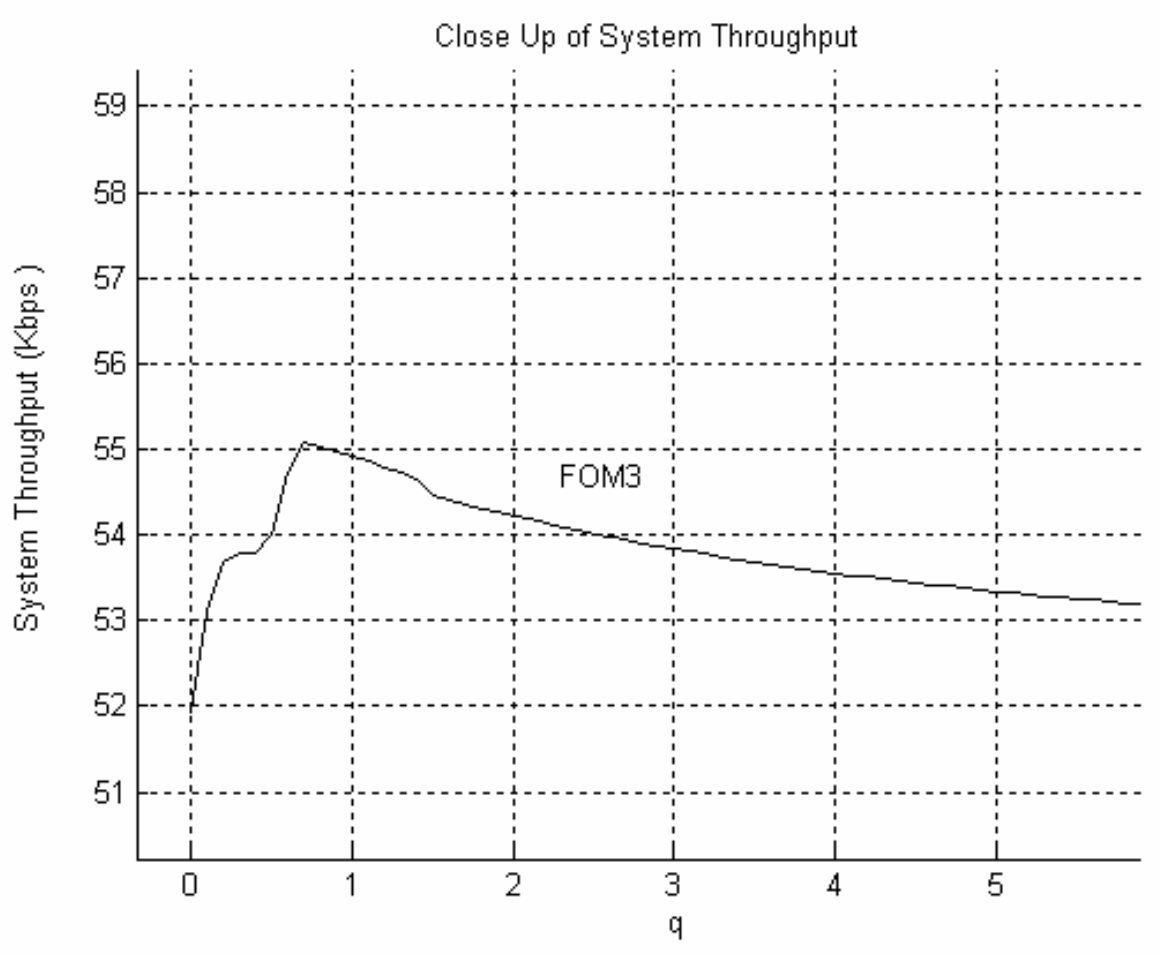

Figure 4-12 Close-up of system throughput of Figure 4-11

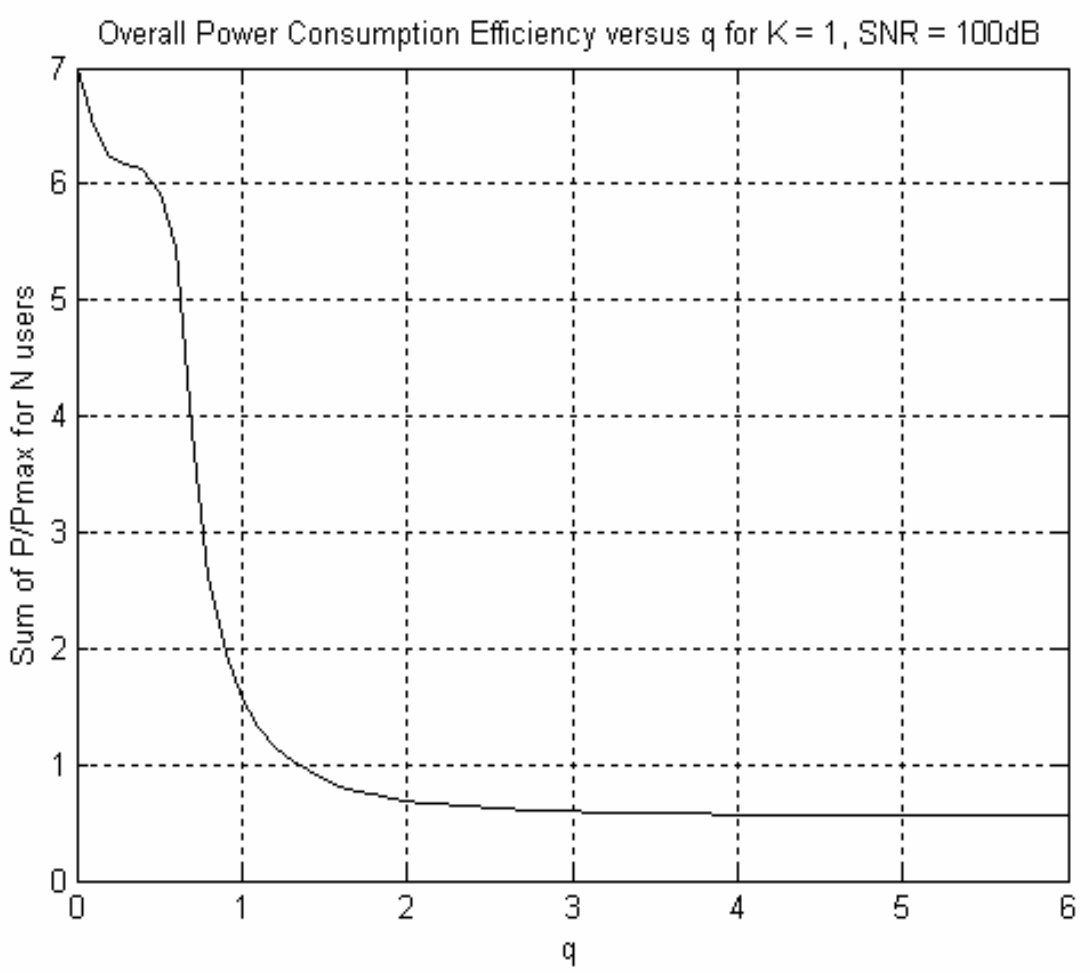

Figure 4-13 Effect of $q$ on power consumption, $\mathrm{SNR}=100 \mathrm{~dB}$ 
At low SNR, we have a noise-limited scenario. A rather extreme case of SNR $=5 \mathrm{~dB}$ is shown in Figure 4-14. The distinguishing factor between the interference and noiselimited cases is that it might not be possible in the noise-limited case, to support some of the higher rates, even at peak power, due to the establishment of a higher "noise floor." As a result, when higher power is penalized more heavily, as is the case for higher values of $q$, there is a significant drop in throughput. Thus, we observe a greater variation in system throughput for the noise-limited case. In Figure 4-14, FOM1 is observed to be maximized for $q=1.6$ and FOM2 for $q=1.3$. However, the drop in throughput is too great at these values. Figure 4-15 suggests that the use of $q \approx 0.6$ results in a small reduction in throughput from its maximum value. This throughput is observed to be achievable within $60 \%$ of the maximum power.

A feature of our model is that it does not deal with hard SINR targets. The softening of targets was mentioned in [63] and the same behavior is apparent from the present discussion. Here, we compare two different situations in which the SINR is adapted so that all users achieve some feasible SINR target, without entering a situation where they all are compelled to transmit at maximum power. As pointed in Section 3.6, this is a common feature of utility-based models.

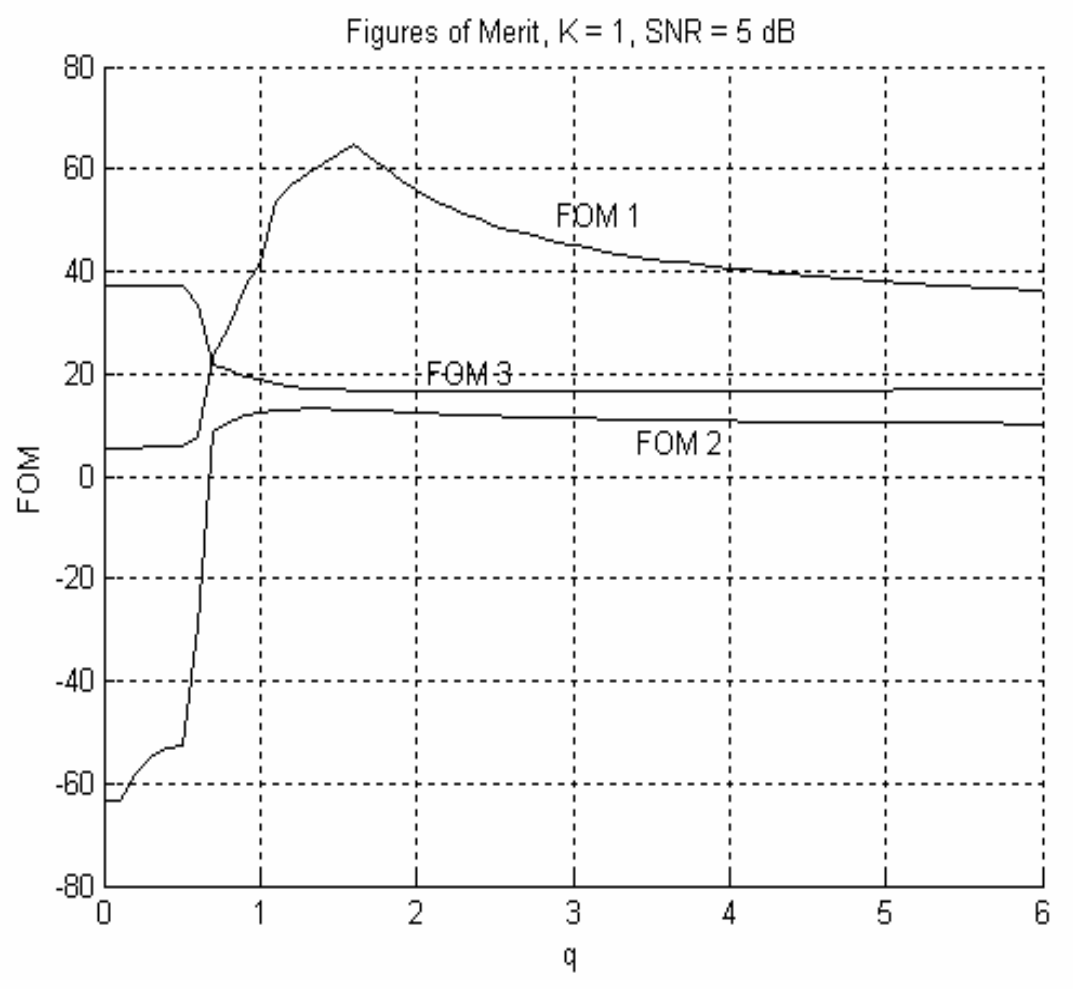

Figure 4-14 Effect of $q$ on FOMs, $\mathrm{SNR}=5 \mathrm{~dB}$ 


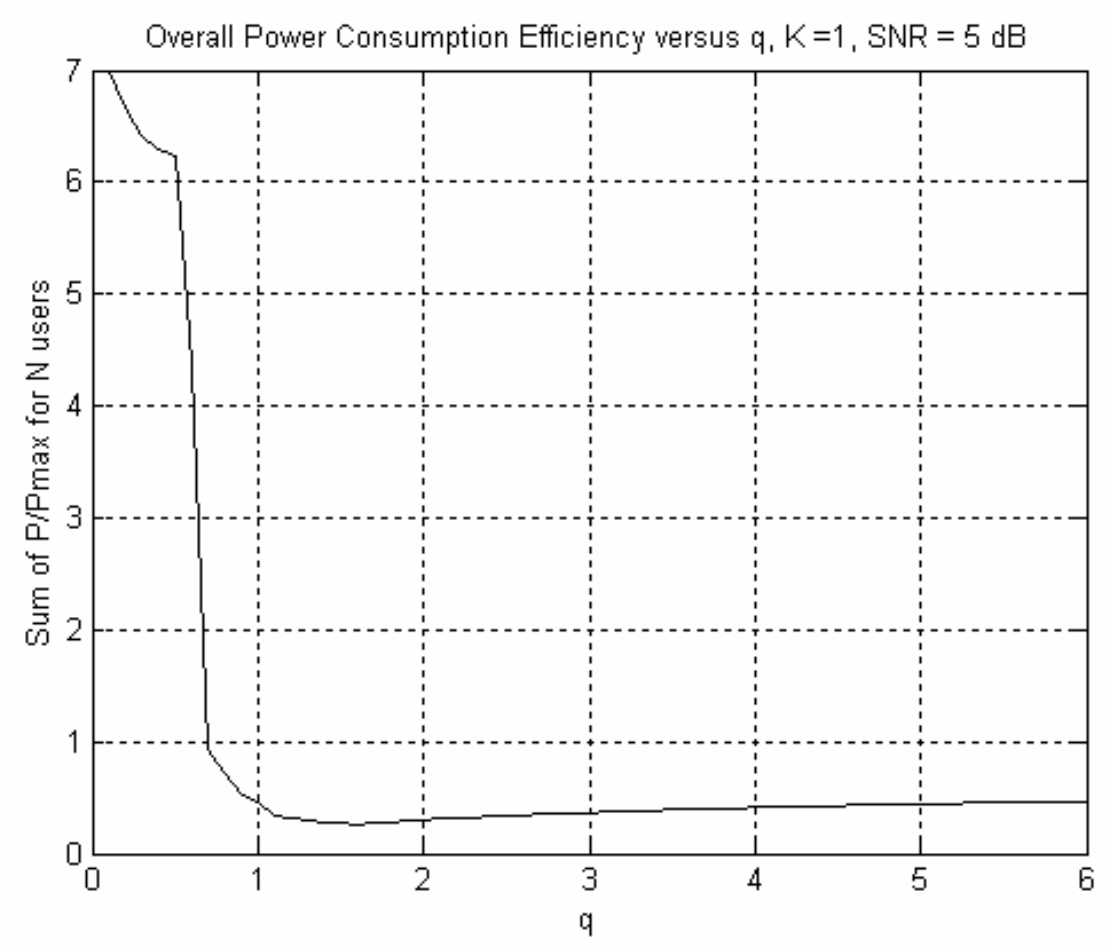

Figure 4-15 Effect of $q$ on power consumption, $\mathrm{SNR}=5 \mathrm{~dB}$

\subsection{Summary}

In this chapter, we presented a game-theoretic framework for analyzing the performance of a system that supports link adaptation. This framework generalized the approaches for solving power control problems in systems without link adaptation (for examples, see Section 3.6). The action set we chose was non-convex, the consequence of which was that we could not show, using standard techniques, that NE exist in the game LAG. This motivated us to develop a heuristic algorithm that iteratively computes each player's bestresponse action until the actions of all players converge. Due to this best-response dynamic, Algorithm LAG converges to a NE. We proved that Algorithm LAG always converges regardless of its initialization. Using a seven-cell system configuration modeled after the downlink of a GPRS system, we showed that Algorithm LAG results in intuitive performance for a given channel realization. Moreover, the NE is not unique. However, our results seemed to indicate that the non-unique equilibria are likely to be close in performance, which is desirable. We introduced a number of FOMs that were used to help tune the parameters of the penalty function, so as to obtain a desired spectral-energy efficiency tradeoff. These results will be consulted in the next chapter when we conduct a performance comparison between Algorithm LAG and several system-theoretic algorithms. 


\section{A Comparison of Game and System- theoretic Results}

There has been little research comparing game-theoretic approaches to solving power control problems with corresponding systems approaches. It is often tempting to trade-off accuracy in utility-based models, in favor of analytic tractability. As an example, consider the utility model [51] described by equations (3-6) - (3-8), in which the utility function was modified to equal zero when power went to zero. Inaccurate modeling may lead to sub-optimal results.

In this chapter, we seek to improve our understanding of the game-theoretic model in Chapter 4 by using a parallel suite of systems techniques to solve the network configuration described in Section 4.5. The development of systems techniques is highlighted in Chapter 2 of this thesis. In that chapter, the power control problem is shown to be related to the dominant eigenvalue of the channel gain matrix of a set of interferers [15]. The evolution of these techniques to embrace data networks is traced through Section 2.4, which discusses power control with multiple rates. The rate a user can achieve is a monotonically increasing function of its received signal to interference and noise ratio (SINR). Hence, it is obvious that selecting rates for the users in a network, or analogously selecting SINR targets corresponding to those rates, is closely tied to power control. The problem considered in Chapter 4 is indeed a power control problem, with multiple rates.

In the following sections, we first formulate the problem of calculating the set of achievable SINR targets that are optimum in the sense that they maximize the sum of targets. Our method differs from others existing in the literature (see Chapter 2) in that it exploits the relationship between the desired targets and the dominant eigenvalue of the channel gain matrix, and uses the subsequently defined "row-sum criterion" in the optimization formulation. A desirable feature of this formulation is that it can be framed as a linear programming problem (LPP), which is easier to solve than a non-linear program. This optimal formulation forms the basis of a systematic algorithm that achieves the same purpose. We also apply the GSPC and GRP algorithms to obtain solutions analogous to the game-theoretic NE.

\subsection{System Model and Problem Definition}

We use a system model and notation consistent with Section 2.1. Let $T$ be the set of $N$ cochannel interfering links. The link gain matrix $\mathbf{G}$ of the system is assumed to remain constant throughout the analysis, as in Chapter 4 . The channel can be assumed static for the duration of convergence of the algorithms that execute on each link $i \in T$. The SINR target of link $i$ is denoted by $\gamma_{i}^{t}$. The vector of targets is defined as $\boldsymbol{\Gamma}^{\mathbf{t}}=\left[\gamma_{1}^{t}, \gamma_{2}^{t}, \ldots, \gamma_{N}^{t}\right]$. 
SINR is defined in Equation (2-1). The vector of SINRs is defined as $\boldsymbol{\Gamma}=\left[\gamma_{1}, \gamma_{2}, \ldots, \gamma_{N}\right]$. Each link $i \in T$ wishes to achieve $\gamma_{i} \geq \gamma_{i}^{t}$. Using Equation (2-1), this condition is equivalent to

$$
P_{i} \geq \sum_{\substack{j=1 \\ j \neq i}}^{N}\left[\frac{\gamma_{i}^{t} G_{i j}}{G_{i i}}\right] P_{j}+\frac{\gamma_{i}^{t} n_{i}}{G_{i i}}
$$

Using the definition for $\mathbf{H}$ in Equation (2-2), and that of $\boldsymbol{\eta}$ in Equation (2-4), we can rewrite Equation (5-1) in matrix form as follows.

$$
(\mathbf{I}-\mathbf{H}) \mathbf{P} \geq \boldsymbol{\eta}
$$

Here, $\mathbf{I}$ is the $N \mathrm{x} N$ identity matrix and $\mathbf{P}$ is the power vector. $\mathbf{P}$ is defined in Section 4.2.1. For simplicity, we denote the set of feasible powers by $\bar{P}$.

From the system performance perspective, it is desirable to find an achievable vector $\Gamma^{t}$ that maximizes the system throughput. Thus, on the surface, it seems logical that system throughput be made the objective function. In our link adaptation problem, the throughput is a function of the discrete-valued Adaptable Link Parameter (ALP), which is defined in Section 4.2. Unfortunately, the optimal allocation of the ALP is unknown at the outset and can be any one of the $|R|^{N}$ ALP vector combinations, where $|R|$ is the cardinality of the ALP set $R$. Thus, it is unclear as to which throughput model we should use in the objective function. However, since throughput is a monotonically increasing function of $\gamma_{i}$, we can alternatively maximize the sum of targets $\gamma_{i}^{t}$. In other words, we define the objective function as follows.

$$
Z=\sum_{i=1}^{N} \gamma_{i}^{t}
$$

The next sub-section presents a method to solve Equation (5-3), subject to a set of derived feasibility constraints.

\subsection{Optimum Target Assignment (OTA)}

We first discuss some important properties of the matrix $\mathbf{H}$.

\section{Lemma 5-1}

1. $\mathbf{H}$ is a non-negative real matrix.

2. $\mathbf{H}$ is an irreducible [76] matrix.

Proof:

1. $\mathbf{H}$ is real and non-negative by its definition, since $\mathbf{G}$ is real and non-negative, and $\Gamma^{t}$ is real and non-negative.

2. It can be observed that no combination of row or column rearrangements can bring $\mathbf{H}$ to a form in which a zero matrix of order $u \times(N-u)$ can be formed, where 
$u$ is an integer such that $0<u<N$. On account of this observation, $\mathbf{H}$ must be an irreducible matrix [76].

Theorem 5-1 is an application of the famous Perron-Frobenius theorem to the matrix $\mathbf{H}$.

\section{Theorem 5-1 Perron-Frobenius Theorem}

The Perron-Frobenius Theorem [76] states that if $\mathbf{H}$ is a real, non-negative and irreducible matrix, then the following is true.

1. The dominant eigenvalue of $\mathbf{H}$, denoted by $\lambda_{1}$, is real and positive.

2. $\lambda_{1}$ is unique, i.e., it is a simple eigenvalue.

3. The eigenvector corresponding to $\lambda_{1}$ is positive, i.e., it does not change its sign.

4. H cannot have two linearly independent non-negative eigenvectors.

In Lemma 5-2, the feasibility condition of the system is derived in terms of the dominant eigenvalue.

\section{Lemma 5-2}

The system (I-H) $\mathbf{P} \geq \boldsymbol{\eta}$ (Equation (5-2)) is feasible when $\lambda_{1}<1$.

Proof:

This property is proved in [77]. For the system $(\mathbf{I}-\mathbf{H}) \mathbf{P} \geq \boldsymbol{\eta}$ to have a solution, the series on the right of Equation (5-4) must converge.

$$
(\mathbf{I}-\mathbf{H})^{-1}=\mathbf{I}+\mathbf{H}+\mathbf{H}^{2}+\ldots
$$

Let $\lambda$ be an eigenvalue of $\mathbf{H}$, with eigenvector $\mathbf{x}$. Equation (5-4) is equivalent to

$$
\left(\mathbf{I}+\mathbf{H}+\mathbf{H}^{2}+\ldots\right) \mathbf{x}=\left(1+\lambda+\lambda^{2}+\ldots\right) \mathbf{x}
$$

The series on the right converges if $\lambda<1$ and the same must hold for any eigenvalue of H. This implies that $\lambda_{1}<1$. This completes the proof.

We now present, without proof, the "row-sum" criterion which places further constraints on $\lambda_{1}$.

\section{Lemma 5-3}

$\lambda_{1}$ must satisfy the condition in Equation (5-6).

$$
\min _{i} R S_{i} \leq \lambda_{1} \leq \max _{i} R S_{i}
$$


Here, $R S_{i}$ is the row-sum of the $i^{\text {th }}$ row of matrix $\mathbf{H}$, given by the expression in Equation $(5-7)$.

$$
R S_{i}=\sum_{j=1}^{N} H_{i j}
$$

Equation (5-6) is known as the row-sum criterion. The equality sign on the right holds if and only if, the upper and lower bounds are equal.

Proof:

See Gantmacher [76].

We now derive a relationship between the row-sum criterion and the objective function in Equation (5-3).

\section{Proposition 5-1}

The objective function $Z$ in Equation (5-3) is maximized when the following condition is satisfied.

$$
R S_{i}=\lambda_{1} \quad \forall i \in T
$$

Proof:

Using the definition of $H_{i}$ in Equation (2-2), we can rewrite Equation (5-7) as shown in Equation (5-9).

$$
R S_{i}=\sum_{\substack{j=1 \\ j \neq i}}^{N} \frac{\gamma_{i}^{t} G_{i j}}{G_{i i}}=\gamma_{i}^{t} \sum_{\substack{j=1 \\ j \neq i}}^{N} \frac{G_{i j}}{G_{i i}}=\gamma_{i}^{t} R S_{i}^{\prime}
$$

Here $R S_{i}^{\prime}$, given in Equation (5-10), is constant for the purpose of the analysis, since $\mathbf{G}$ is assumed constant at the outset of Section 5.1.

$$
R S_{i}^{\prime}=\sum_{\substack{j=1 \\ j \neq i}}^{N} \frac{G_{i j}}{G_{i i}}
$$

From Equation (5-3) and Equation (5-9), we have an expression for the objective function $Z$ in terms of the row-sums.

$$
Z=\sum_{i=1}^{N} \frac{R S_{i}}{R S_{i}^{\prime}}
$$

Let $k=\arg \max _{i \in T} R S_{i}$ and $j=\arg \min _{i \in T} R S_{i}$. It follows from Lemma 5-3 that

$$
R S_{j} \leq \lambda_{1} \leq R S_{k}
$$

Let us denote the optimal value of the objective function by $Z^{\text {opt }}$. Let this value be achieved when $\lambda_{1}=\tilde{\lambda}_{1}$ and $\gamma_{i}^{t}=\tilde{\gamma}_{i}^{t}$. Denoting the corresponding row-sums by $R S_{i}^{o p t}$, we have 


$$
Z^{\text {opt }}=\sum_{i=1}^{N} \tilde{\gamma}_{i}^{t}=\sum_{i=1}^{N} \frac{R S_{i}^{o p t}}{R S_{i}^{\prime}}
$$

Equation (5-11) is maximized if and only if, $R S_{i}=R S_{k}$, for all $i$. Hence $Z=Z^{\text {opt }}$ if and only if, $R S_{i}^{\text {opt }}=R S_{k}^{\text {opt }}$, for all $i$. Then, $R S_{j}^{\text {opt }}=R S_{k}^{o p t}$, which implies, from Equation (5-12), that $R S_{i}^{o p t}=\tilde{\lambda}_{1}, \forall i \in T$. This completes the proof.

Lemma 5-2 states that the maximum value of $\lambda_{1}$ for the system to be feasible is 1 , but this may not necessarily be the value of $\lambda_{1}$ that achieves the targets that maximize $Z$.

Note that in Equation (2-4) $\eta_{i}=\left\{\frac{\gamma_{i}^{t} n_{i}}{G_{i i}}\right\}$. It is clear that the contribution of noise increases in direct proportion with SINR target. In the purely interference-limited case, the optimum system throughput would correspond to $\lambda_{1}=1$. However, in general, the dominant eigenvalue is constrained by noise, which leads us to propose the following.

\section{Proposition 5-2}

There exists $\mathbf{P} \in \bar{P}$ such that $\mathbf{P}$ is an eigenvector of $(\mathbf{I}-\mathbf{H})$, if and only if $\mathbf{P}_{\max } \geq \boldsymbol{\eta}$.

\section{Proof:}

Theorem 5-1 states that the eigenvector corresponding to $\lambda_{1}$ is positive (Theorem 5-1.3) and unique (Theorem 5-1.4). If there exists an eigenvector $\mathbf{P}_{1}$ corresponding to the eigenvalue $\left(1-\lambda_{1}\right)$ of $(\mathbf{I}-\mathbf{H})$, then, using Equation $(5-2)$, we have $\left(1-\lambda_{1}\right) \mathbf{P}_{1} \geq \boldsymbol{\eta}$. Rearranging, we get Equation (5-14).

$$
\mathbf{P}_{1} \geq \frac{\boldsymbol{\eta}}{\left(1-\lambda_{1}\right)}
$$

Lemma 5-2 and Theorem 5-1 (see 5-1.1) imply that $0 \leq \lambda_{1}<1$. It follows that

$$
\boldsymbol{\eta} \leq \frac{\boldsymbol{\eta}}{1-\lambda_{1}}<\infty
$$

For feasible $\mathbf{P}_{1}$, we must have $\mathbf{P}_{1} \leq \mathbf{P}_{\max }$. Combining this result with Equation (5-14) and Equation (5-15), we must have $\mathbf{P}_{\max } \geq \boldsymbol{\eta}$. This completes the proof.

Proposition 5-2 establishes the necessary condition for the dominant eigenvector of $(\mathbf{I}-\mathbf{H})$ to be a feasible solution to Equation (5-2). We now derive the constraints of our optimization problem. From Proposition 5-2, we can develop the following constraint on $\lambda_{1}$. Using Equation (5-14) and Equation (2-4), we have 


$$
\lambda_{1} \leq 1-\frac{\gamma_{i}^{t} n_{i}}{G_{i i} P_{\max }}, \forall i \in T
$$

Proposition 5-1 suggests that $\lambda_{1}$ must be maximized. However, from Lemma 5-3, $\lambda_{1}$ must always be bounded by the maximum row sum but is permitted to be greater than all the other row sums. By this reasoning, we have our second constraint, as expressed in Equation (5-17).

$$
\lambda_{1} \geq \gamma_{i}^{t} \sum_{\substack{j=1 \\ j \neq i}}^{N} \frac{G_{i j}}{G_{i i}}, \forall i \in T
$$

Proposition 5-1 shows that, at the solution, Equation (5-17) will become an equality. Otherwise, at all times, we will have $\lambda_{1}=\max _{i \in T} R S_{i}$. In Definition 5-1, we formally define the optimization problem using the objective in Equation (5-3), subject to the constraints in Equation (5-16) and Equation (5-17).

\section{Definition 5-1 Max-Sum of SINR Targets}

$$
\max _{\Gamma^{t}, \lambda_{1}}\left(Z=\sum_{i=1}^{N} \gamma_{i}^{t}\right)
$$

subject to
a. $\quad \lambda_{1} \leq 1-\frac{\gamma_{i}^{t} n_{i}}{G_{i i} P_{\max }}, \forall i \in T$.
b. $\quad \lambda_{1} \geq \gamma_{i}^{t} \sum_{\substack{j=1 \\ j \neq i}}^{N} \frac{G_{i j}}{G_{i i}}, \forall i \in T$.
c. $0 \leq \lambda_{1}<1, \quad \mathbf{0} \leq \boldsymbol{\Gamma}^{t}$.

Our problem formulation is an LPP in the independent variables $\Gamma^{t}$ and $\lambda_{1}$. It can be solved using well known linear programming techniques [78].

\subsection{Stepwise Rate Removals Algorithm}

Based on the work in Section 5.2, we propose a heuristic algorithm that commences with a potentially infeasible system and uses the row-sum criterion to bring it to feasibility. This algorithm, called Stepwise Rate Removals (SRR), is centralized, and begins by assigning maximum possible SINR targets to all users. Generally, this assignment will be infeasible. SRR iteratively calculates the eigenvalue of the system, and reduces the rates of those users demanding unreasonably high rates, until feasibility is achieved. The feasibility bound on the eigenvalue is derived using Equation (5-16). The row-sum criterion of Equation (5-17) is used to identify the user most likely to make the system infeasible. 


\section{Definition 5-2 Stepwise Rate Removals Algorithm}

The SRR algorithm is now presented and uses the notation developed in the preceding sections. We define a discrete-valued set of targets $\bar{\Gamma}=\left\{\bar{\gamma}^{1}, \bar{\gamma}^{2}, \ldots, \bar{\gamma}^{\bar{\Gamma} \mid}\right\}$ such that $\gamma_{i}^{t} \in \bar{\Gamma}, \forall i \in T$. Furthermore, we require that the targets be in a geometric progression, i.e., $\frac{\bar{\gamma}^{1}}{\bar{\gamma}^{2}}=\frac{\bar{\gamma}^{2}}{\bar{\gamma}^{3}}=\ldots=\frac{\bar{\gamma}^{|\bar{\Gamma}|-1}}{\bar{\gamma}^{|\bar{\Gamma}|}}=f$. The variable $f$ is the rate removal factor and must be strictly less than one.

1. Start.

2. Initialization

a. Iteration $k=0$.

b. Initialize targets to maximum: $\gamma_{i}^{t}(0)=\max \{\bar{\Gamma}\}=\bar{\gamma}^{|\bar{\Gamma}|}, \forall i \in T$.

3. While $\lambda_{1}>1-\max _{i \in T}\left\{\frac{\eta_{i}}{P_{\max }}\right\}$

a. Find row with maximum row-sum.

$$
j=\arg \max _{i \in T} R S_{i}
$$

b. Scale down the target of that row. In other words, remove a rate.

$$
\gamma_{j}^{t}(k+1)=f \gamma_{j}^{t}(k)
$$

c. Recalculate row $j$ of $\mathbf{H}$ and $\eta_{j}$ from Equation (2-2) and Equation (2-4) respectively.

d. $k=k+1$.

4. Stop.

We now show that the SRR algorithm is guaranteed to converge.

\section{Proposition 5-3}

The Stepwise Rate Removals algorithm always converges.

Proof:

At iteration $k$, let $q_{i}(k)$ be the scalar that counts the number of times each user $i$ has demanded an infeasible rate; in other words, $q_{i}(k)$ counts the instances when $\lambda_{1}>1-\max _{l \in T}\left\{\frac{\eta_{l}}{P_{\max }}\right\}$ and $i=\arg \max _{l \in T} R S_{l}$. At iteration $k$ of SRR, we must have $\gamma_{i}^{t}(k)=f^{q_{i}(k)} \gamma_{i}^{t}(0), \forall i \in T$. As $k \rightarrow \infty, \quad q_{i}(k) \rightarrow \infty$. Hence, $\gamma_{i}^{t}(k)=0, \forall i$. Thus, as $k \rightarrow \infty, \mathbf{H}=\mathbf{0}_{N \times N}$, and $\boldsymbol{\eta}=\mathbf{0}_{N \times 1}$, where $\mathbf{0}$ is the null matrix. Then, $\lambda_{1}=0$, which being less than 1, will result in termination of the SRR algorithm at step 3. 
It is not straightforward to calculate $\lambda_{1}$, the dominant eigenvalue of $\mathbf{H}$, in a distributed manner. This implies that the SRR algorithm is centralized and not convenient for implementation. However, it serves as a good substitute for the LPP formulated in Section 5.2. The results presented in Section 5.4 substantiate this observation. Distributed or semi-distributed techniques already exist for finding the feasible achievable SINRs in a network. A good example is Generalized Selective Power Control (GSPC) [21].

\subsection{Simulation Results and Comparison}

To facilitate comparison between the results in Chapter 4 and this section, we use the system description for a GPRS network presented in Section 4.5.1. In the present section, we compare the performance achieved by the following techniques for assigning rates.

1. The optimum target assignment (OTA) calculated by the optimization formulation in Section 5.2.

2. The Stepwise Rate Removals (SRR) algorithm of Section 5.3.

3. Generalized Selective Power Control with Gradual Rate Removals (GSPC-GRR), which is proposed in [21]. Our own implementation of this algorithm is described in Section 2.4.1.

4. The Greedy Rate Packing (GRP) algorithm, that also appears in [21]. Our own implementation of this algorithm is described in Section 2.4.1.

5. The game-theoretic Algorithm LAG, described in Section 4.4.

We consider the interference-limited and noise-limited scenarios, the results for which are presented via a series of tables, labeled Table 5-1 to Table 5-10. Each table corresponds to results obtained by using one of the techniques mentioned in the foregoing paragraph, and is labeled accordingly. The tables state the achievable SINR targets, GPRS coding schemes (CS) allocated, the system throughput and transmitter power for each link. CS selection in all methods, except Algorithm LAG, is accomplished by mapping SINR to CS according to the rules described in Table 4-5. These rules ensure that the maximum possible throughput is achieved for the given SINR. SRR and GSPC are implemented using a logarithmically increasing sequence of discrete SINRs, belonging to the set $\{0,1,2, \ldots, 50\} \mathrm{dB}$. The maximum value of $50 \mathrm{~dB}$ is arbitrary. In GRP, we limit the SINR to occupy the interval $[0,30] \mathrm{dB}$. The penalty-function parameters of Algorithm LAG are selected using values recommended by the tuning exercise carried out in Section 4.5.3.2 (see Figure 4-11 - Figure 4-15). A value of $q=2$ is chosen for the interference-limited scenario (Section 5.4.1) and $q=0.6$ for the noise-limited scenario (Section 5.4.2). The results are then discussed in Section 5.4.3. 


\subsubsection{Interference-limited Scenario}

For the interference-limited scenario, the signal to noise ratio (SNR) is set to $100 \mathrm{~dB}$.

Table 5-1 Optimum Target Assignment $(\mathrm{SNR}=100 \mathrm{~dB})$

\begin{tabular}{|l|c|c|c|c|c|c|c|}
\hline User & 1 & 2 & 3 & 4 & 5 & 6 & 7 \\
\hline SINR $(\mathrm{dB})$ & 6.53 & 38.77 & 8.77 & 17.80 & 8.77 & 8.77 & 8.77 \\
\hline CS & 1 & 4 & 2 & 3 & 2 & 2 & 2 \\
\hline Power(mW) & 0.504 & 0.504 & 0.504 & 0.504 & 0.504 & 0.504 & 0.504 \\
\hline
\end{tabular}

Table 5-2 Stepwise Rate Removal $(\mathrm{SNR}=100 \mathrm{~dB})$

\begin{tabular}{|l|c|c|c|c|c|c|c|}
\hline User & 1 & 2 & 3 & 4 & 5 & 6 & 7 \\
\hline SINR (dB) & 6 & 38 & 9 & 18 & 9 & 9 & 9 \\
\hline CS & 1 & 4 & 2 & 3 & 2 & 2 & 2 \\
\hline Power(mW) & $0.43 \mathrm{e}-6$ & $0.40 \mathrm{e}-6$ & $0.49 \mathrm{e}-6$ & $0.50 \mathrm{e}-6$ & $0.51 \mathrm{e}-6$ & $0.51 \mathrm{e}-6$ & $0.49 \mathrm{e}-6$ \\
\hline
\end{tabular}

Table 5-3 Generalized Selective Power Control $(\mathrm{SNR}=100 \mathrm{~dB})$

\begin{tabular}{|l|c|c|c|c|c|c|c|}
\hline User & 1 & 2 & 3 & 4 & 5 & 6 & 7 \\
\hline SINR (dB) & 4.59 & 49.02 & 3.13 & 11.23 & 3.24 & 3.34 & 3.00 \\
\hline CS & 1 & 4 & 1 & 3 & 1 & 1 & 1 \\
\hline Power(mW) & $2.43 \mathrm{e}-6$ & $1.775 \mathrm{e}-5$ & $1.62 \mathrm{e}-6$ & $0.52 \mathrm{e}-6$ & $0.6 \mathrm{e}-6$ & $0.7 \mathrm{e}-5$ & $1.58 \mathrm{e}-6$ \\
\hline
\end{tabular}

Table 5-4 Greedy Rate Packing $(\mathrm{SNR}=100 \mathrm{~dB})$

\begin{tabular}{|c|c|c|c|c|c|c|c|}
\hline User & 1 & 2 & 3 & 4 & 5 & 6 & 7 \\
\hline SINR (dB) & 3.44 & 30.00 & 2.63 & 29.78 & -1.91 & 4.15 & 5.56 \\
\hline $\mathrm{CS}$ & 1 & 4 & 1 & 4 & 1 & 1 & 1 \\
\hline Power(mW) & $7.72 \mathrm{e}-7$ & $1.25 \mathrm{e}-7$ & $6.03 e-7$ & $7.79 e-6$ & $2.17 \mathrm{e}-7$ & $2.92 \mathrm{e}-7$ & $3.83 e-7$ \\
\hline
\end{tabular}

Table 5-5 Game Theory: Algorithm LAG (SNR $=100 \mathrm{~dB}, q=2, K=1)$

\begin{tabular}{|l|c|c|c|c|c|c|c|}
\hline User & 1 & 2 & 3 & 4 & 5 & 6 & 7 \\
\hline SINR (dB) & 6.63 & 33.34 & 10.38 & 16.85 & 9.53 & 9.27 & 10.04 \\
\hline CS & 1 & 4 & 2 & 3 & 2 & 2 & 2 \\
\hline Power(mW) & 1.01 & $3.18 \mathrm{e}-1$ & 1.16 & $8.71 \mathrm{e}-1$ & 1.19 & 1.2 & 1.18 \\
\hline \multicolumn{7}{l}{ System Throughput (kbps): 54.22 } \\
\hline
\end{tabular}




\subsubsection{Noise-limited Scenario}

For the noise-limited scenario, the SNR is set to $5 \mathrm{~dB}$.

Table 5-6 Optimum Target Assignment $(\mathrm{SNR}=5 \mathrm{~dB})$

\begin{tabular}{|l|c|c|c|c|c|c|c|}
\hline User & 1 & 2 & 3 & 4 & 5 & 6 & 7 \\
\hline SINR (dB) & 1.23 & 33.48 & 3.48 & 12.51 & 3.48 & 3.48 & 3.48 \\
\hline CS & 1 & 4 & 1 & 3 & 1 & 1 & 1 \\
\hline Power(mW) & 7.064 & 9.692 & 9.692 & 9.692 & 9.692 & 9.692 & 9.692 \\
\hline
\end{tabular}

Table 5-7 Stepwise Rate Removal $(\mathrm{SNR}=5 \mathrm{~dB})$

\begin{tabular}{|l|c|c|c|c|c|c|c|}
\hline User & 1 & 2 & 3 & 4 & 5 & 6 & 7 \\
\hline SINR (dB) & 1 & 33 & 3 & 12 & 3 & 3 & 3 \\
\hline CS & 1 & 4 & 1 & 3 & 1 & 1 & 1 \\
\hline Power(mW) & 6.32 & 8.37 & 8.366 & 8.311 & 8.366 & 8.37 & 8.37 \\
\hline
\end{tabular}

Table 5-8 Generalized Selective Power Control $(\mathrm{SNR}=5 \mathrm{~dB})$

\begin{tabular}{|l|c|c|c|c|c|c|c|}
\hline User & 1 & 2 & 3 & 4 & 5 & 6 & 7 \\
\hline SINR (dB) & 2 & 33 & 3 & 12 & 3 & 3 & 3 \\
\hline CS & 1 & 4 & 1 & 3 & 1 & 1 & 1 \\
\hline Power(mW) & 8.01 & 8.524 & 8.521 & 8.464 & 8.52 & 8.524 & 8.524 \\
\hline
\end{tabular}

Table 5-9 Greedy Rate Packing $(\mathrm{SNR}=5 \mathrm{~dB})$

\begin{tabular}{|l|c|c|c|c|c|c|c|}
\hline User & 1 & 2 & 3 & 4 & 5 & 6 & 7 \\
\hline SINR (dB) & 2.66 & 30.00 & 3.70 & 12.57 & 3.53 & 3.53 & 3.70 \\
\hline CS & 1 & 4 & 1 & 3 & 1 & 1 & 1 \\
\hline Power(mW) & 9.56 & 4.47 & 10.0 & 10.0 & 10.0 & 10.0 & 10.0 \\
\hline
\end{tabular}

Table 5-10 Game Theory: Algorithm LAG $(\mathrm{SNR}=5 \mathrm{~dB}, q=0.6, K=1)$

\begin{tabular}{|c|c|c|c|c|c|c|c|}
\hline User & 1 & 2 & 3 & 4 & 5 & 6 & 7 \\
\hline SINR (dB) & $-\infty$ & 26.64 & 3.27 & 13.15 & 2.91 & 3.03 & 3.39 \\
\hline $\mathrm{CS}$ & 1 & 4 & 1 & 3 & 1 & 1 & 1 \\
\hline Power(mW) & 0 & 1.8 & 8.0 & 10 & 7.7 & 7.8 & 8.1 \\
\hline
\end{tabular}




\subsubsection{Discussion}

We make some general observations from the results presented in Table 5-1 to Table 5-10. To aid this discussion, we calculate the corresponding FOM values (Section 4.5.2) and present them in Table 5-11. FOM 3 is the system throughput which, by virtue of already being included in the earlier results, is not restated here.

Table 5-11 Comparison of results based on FOM 1 and FOM 2 values

\begin{tabular}{|l|c|c|c|c|}
\hline \multirow{2}{*}{ Technique } & \multicolumn{2}{|c|}{ SNR $=100 \mathrm{~dB}$} & \multicolumn{2}{c|}{ SNR $=5 \mathrm{~dB}$} \\
\cline { 2 - 5 } & FOM 1 & FOM 2 & FOM 1 & FOM 2 \\
\hline OTA & 147.19 & 46.86 & 5.60 & -57.12 \\
\hline SRR & $1.5709 \mathrm{e} 8$ & 52.31 & 6.22 & -45.95 \\
\hline GSPC & $1.1686 \mathrm{e} 7$ & 36.81 & 6.02 & -49.29 \\
\hline GRP & $4.1249 \mathrm{e} 7$ & 42.00 & 5.81 & -54.74 \\
\hline Algorithm LAG & 78.25 & 44.27 & 7.73 & -28.77 \\
\hline
\end{tabular}

Table 5-11 seems to suggest that, on the surface, SRR performs best in the interferencelimited scenario and Algorithm LAG does likewise in the noise-limited scenario. However, this does not present the entire picture and a more thorough examination of results is required. By studying system throughput, we note that although OTA is designed to maximize the sum of SINRs, it need not be guaranteed to maximize system throughput. The reason, which is evident from performance curves such as Figure 1-2, is that throughput saturates at high values of SINR. Beyond a certain point, the power increment required to achieve even small increases in throughput is large, causing more interference. GRP and GSPC tend to favor users that experience good channel conditions to a greater extent than any of other schemes studied. This is not the fairest way of allocating resources, since users in poor or even moderate conditions might tend to suffer from throughput starvation. Specifically, as many as 5 out of 7 users are assigned CS-1 in both Table 5-3 (GSPC) and Table 5-4 (GRP). The FOM calculations are unable to reflect this fact. By punishing excessive power consumption, and at the same time attempting to maximize throughput, Algorithm LAG generates results that are fair. Like OTA and SRR, Algorithm LAG results in only a single user being assigned CS-1.

Comparing the power consumption figures, Algorithm LAG and OTA result in higher power consumption than necessary to achieve the observed system throughput. As the targets increase, the excess power required to achieve them grows faster than the gain in throughput that is achieved. However, at SNR $=100 \mathrm{~dB}$, it is obvious that by scaling down all the powers by some common factor, similar system throughput can still be attained. The SRR scheme, which we recall is derived from OTA, effectively demonstrates this point (compare Table 5-1 to Table 5-2). Note, however, that if it were possible to introduce more spectral efficiency by switching to a higher modulation scheme when conditions permitted, these throughput saturation effects could be avoided. Yet, in the SNR $=100 \mathrm{~dB}$ scenario, the maximum PCC (Section 4.5.2) is still approximately less than $10 \%$ for all schemes. Since the top priority for the downlink is to maximize throughput, the observed power consumption might still be reasonable. Despite its apparent inefficiency, Algorithm LAG results in the highest system throughput for the 
$\mathrm{SNR}=100 \mathrm{~dB}$ scenario. It is yet possible to ameliorate its slightly disappointing power consumption by increasing the penalty function parameter $K$. The dramatic improvement in the power consumption of Algorithm LAG is reflected in Table 5-12. This table charts the descent of the maximum PCC (expressed as a percentage) of the system. Significantly, this improvement is not accompanied by any perceptible decrease in the system throughput.

Table 5-12 Impact of varying $K$ on PCC

\begin{tabular}{|c|c|c|}
\hline$K$ & Max PCC $<$ & System Throughput \\
\hline 1 & $10 \%$ & 54.22 \\
\hline 10 & $4 \%$ & 54.22 \\
\hline 1000 & $0.4 \%$ & 54.18 \\
\hline 100000 & $0.04 \%$ & 54.24 \\
\hline
\end{tabular}

In general, Algorithm LAG, OTA and SRR achieve good tradeoffs between throughput and power consumption. In the interference-limited scenario, SRR performs the best overall. The FOM calculations in Table 5-11 support this observation. GRP tends to minimize the power consumption in the interference-limited scenario. However, it achieves the highest throughput amongst the schemes considered for the noise-limited scenario, but consumes the maximum power in doing so. It is worth noting that Algorithm LAG is the most power efficient in the noise-limited scenario. At the same time, it does not excessively compromise throughput. This is due to the flexibility in tuning the parameters of Algorithm LAG to achieve the best throughput-power consumption tradeoff. The FOM calculations in Table 5-11, once again substantiate this observation. Amongst the techniques being compared here, this feature is unique to Algorithm LAG and is an advantage of employing a game-theoretic approach.

From this discussion, we suggest a simple solution for introducing fairness in GSPC. We propose restricting the maximum value of the set of targets to a lower value than before. For the example we are considering, $30 \mathrm{~dB}$ might be an appropriate value, based on the knowledge that the same worked well for the GRP simulations. The impact of this target limitation is reflected in the decisive improvement of approximately $9 \mathrm{kbps}$ in the resulting system throughput, seen in Table 5-13, when compared to Table 5-3. In addition, there are only 3 users, as compared to the earlier 5 , that are assigned the minimum rate by GSPC. The performance of the users that benefited most in Table 5-3 is not compromised by target limitation, even though it has improved the situation for at least three users, viz. users 1,3 and 4 . Thus, target limitation has made GSPC clearly fairer. 
Table 5-13 GSPC with Target Limitation $(\mathrm{SNR}=100 \mathrm{~dB}$, Max. target $=30 \mathrm{~dB})$

\begin{tabular}{|l|c|c|c|c|c|c|c|}
\hline User & 1 & 2 & 3 & 4 & 5 & 6 & 7 \\
\hline SINR (dB) & 8.51 & 32.08 & 7.32 & 23.56 & 3.58 & 3.71 & 3.00 \\
\hline CS & 2 & 4 & 2 & 4 & 1 & 1 & 1 \\
\hline Power(mW) & $6.84 \mathrm{e}-7$ & $9.83 \mathrm{e}-8$ & $4.89 \mathrm{e}-7$ & $1.55 \mathrm{e}-6$ & $2.13 \mathrm{e}-7$ & $1.25 \mathrm{e}-7$ & $1.01 \mathrm{e}-7$ \\
\hline
\end{tabular}

To obtain an unbiased performance comparison between all the techniques considered here, we run several simulations for each of them with random mobile locations within a seven-cell configuration. In addition, the path gain on each link also includes a lognormal shadowing component with an $8 \mathrm{~dB}$ standard deviation. These are the only changes to the model proposed in Section 4.5.1. We compare the downlink system throughput obtained in an interference-limited scenario (SNR = $100 \mathrm{~dB}$ ). In Figure 5-1, we plot the empirical cumulative distribution function (CDF) of system throughput for GSPC-GRR, GRP, SRR, OTA and Algorithm LAG.

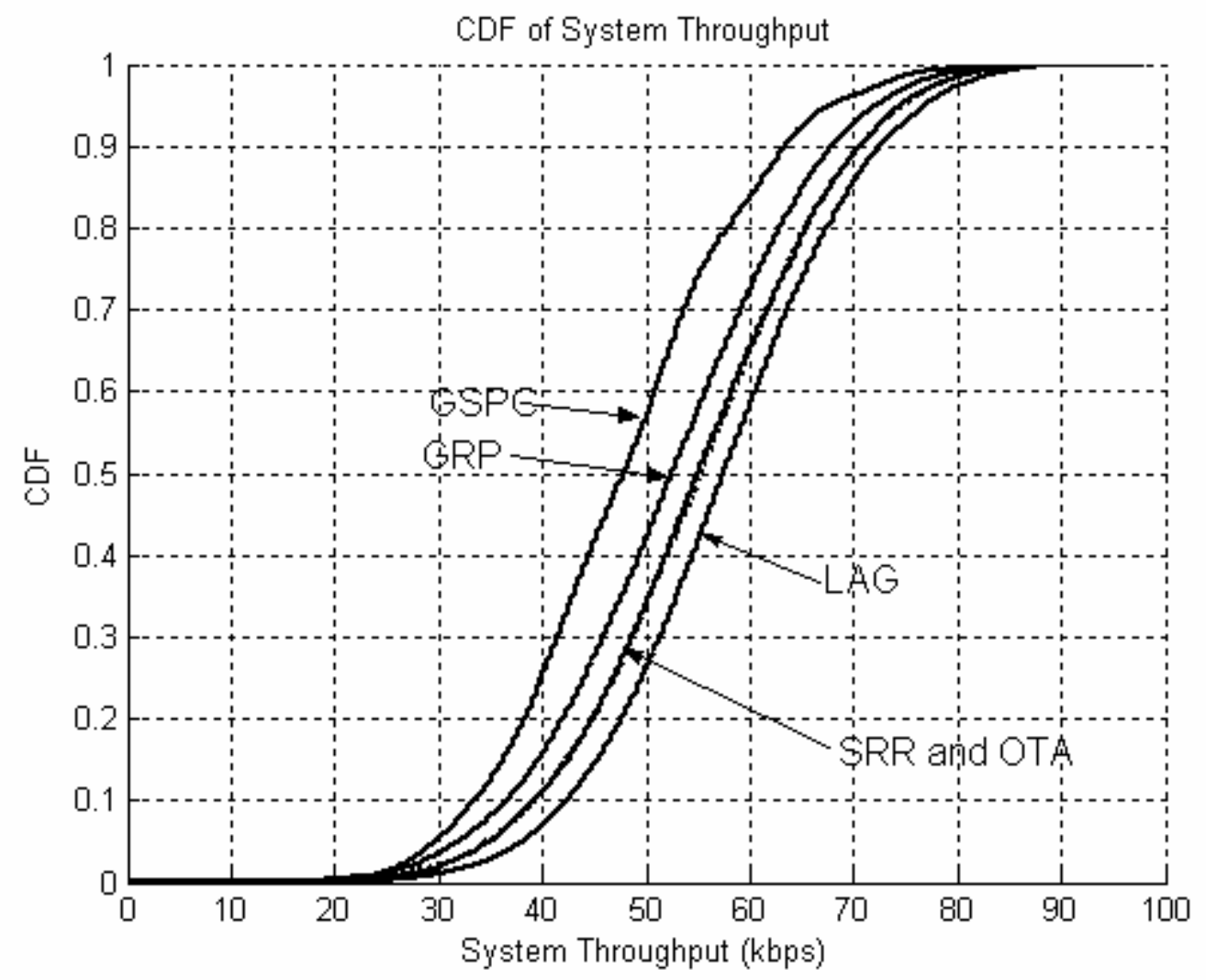

Figure 5-1 Performance comparison of GSPC-GRR, GRP, SRR, OTA and Algorithm LAG using CDF of system throughput (kbps) for the interference-limited scenario (SNR $=100 \mathrm{~dB}$ ). Note that the performances of SRR and OTA are nearly identical.

The improvement in system throughput - a measure of system capacity improvement which Algorithm LAG achieves over the other techniques we have considered is clearly evident in Figure 5-1. For example, if we consider the fraction of the time system throughput exceeds $60 \mathrm{kbps}$, we notice that this works out to $41 \%$ for Algorithm LAG, 
which is clearly greater than the $35 \%$ for OTA and SRR, $27 \%$ for GRP and $16 \%$ for GSPC-GRR. The mean percentage system capacity improvement that Algorithm LAG achieves over its competitors is calculated in Table 5-14, using average system throughput as a measure of capacity. The nearest competitors are OTA and SRR. Algorithm LAG obtains over 4\% improvement over these methods. However, note that Algorithm LAG is completely distributed, while OTA and SRR are centralized. This observation favors Algorithm LAG. Furthermore, the improvement over GRP, which is centralized, is nearly 10\%, while over GSPC-GRR, which is distributed, it approaches $20 \%$.

Table 5-14 Percentage System Capacity Improvement obtained by Algorithm LAG

\begin{tabular}{|c|c|}
\hline $\begin{array}{c}\text { Compared } \\
\text { to }\end{array}$ & $\begin{array}{c}\text { \% Capacity } \\
\text { Improvement }\end{array}$ \\
\hline GSPC-GRR & 18.35 \\
\hline GRP & 9.75 \\
\hline OTA & 4.36 \\
\hline SRR & 4.36 \\
\hline
\end{tabular}

\subsection{Summary}

This chapter drew revealing performance comparisons between game and systemtheoretic techniques. An optimization approach using systems techniques (OTA and SRR), which attempts to maximize the sum of SINR targets of the system, was first presented. Next, the performance of this approach was compared with GSPC-GRR, GRP and Algorithm LAG. The comparison was based on system throughput on the downlink, power consumption, fairness and flexibility. The main conclusion was that Algorithm LAG achieves a capacity gain over all competing techniques. The performances of OTA and SRR were within $5 \%$ of Algorithm LAG. However, neither of these is distributed. In terms of power consumption, we initially observed that Algorithm LAG consumes more power than necessary, for an interference-limited scenario. The increase of the penalty function weighting factor $K$ was found to decrease power consumption by a few orders of magnitude, without compromising throughput. In terms of fairness, Algorithm LAG was shown to achieve a fairness that is matched by OTA and SRR, but not by GRP and GSPC-GRR. The latter two are greedy in that they assign maximum resources to the few users in the most favorable conditions, to the extent that several users in less favorable conditions are starved. We finally mention that the comparison exercise carried out here is not conducted in other game-theoretic work. 


\section{Conclusions}

Game theory is an exciting new approach for solving RRM problems. While systems theory, which we vaguely define in Chapter 1, has been hitherto favored for this task, the complex nature of $3 \mathrm{G}$ systems has prompted researchers to take a fresh look at available solution methodologies ${ }^{8}$. To assess the significance and usefulness of game theory in wireless communications, it helps to understand the characteristics of systems approaches that have rendered them so attractive to the wireless engineer. Foremost amongst these considerations is the formidable collection of mathematical concepts from fields such as linear algebra, optimization, queuing theory, statistics and calculus that lend themselves to the analysis of almost any RRM problem. The versatility of these techniques is clearly demonstrated in [4]. Equally important is the readiness with which implementationfriendly distributed algorithms emerge from system-level analysis. In power control literature, excellent examples of implementations arising from optimal problem formulations can be found in [18], [20], and [21]. In our opinion, these qualities make systems techniques almost indispensable.

Analogously, game theory has proven itself to be just as important to economists. This has resulted in the establishment of an equally vast body of literature in game theory. The realization that several problems in wireless networks can be viewed as non-cooperative games has spawned numerous attempts at game-theoretic system analysis. The rationality assumptions (Section 3.1) of game theory are conveniently valid when applied to computing machines. In no other area of wireless communications is the popularity of game-theoretic analysis more evident than in power control [48] [51]. This thesis is simply an extension of these approaches.

Our main contribution is the generalization of power control games to incorporate link adaptation, as discussed in Chapter 4. This resulted in a modification of the typical action set of a power control game to include a discrete-valued Adaptable Link Parameter (ALP), in addition to power. The impact of this change is that it becomes impossible to guarantee the existence of a Nash Equilibrium (NE) in the network using well-known NE existence results. Resorting to heuristic approaches, we construct an iterative algorithm that discovers a NE and show that it - the algorithm - always converges. The simulation results arising from this intuitive approach are themselves satisfyingly intuitive. More significantly, the performance of this algorithm is shown to be favorable compared to analogous system-theoretic schemes. As indicated in Section 5.4.3, the game-theoretic approach stands out in terms of fairness and flexibility. In an interference-limited scenario, it also achieves a downlink system throughput improvement of $4-20 \%$ over competing schemes, with reasonable power consumption. We notice that the important task of performance validation and evaluation is missing in most game-theoretic analyses. However, we stress that the importance of this exercise in benchmarking the efficacy of

\footnotetext{
${ }^{8}$ Chapter 3 provides a comprehensive summary of game-theoretic applications in power control
} 
game theory must not be underestimated. The ultimate success of game-theoretic analysis will rely on such comparisons.

Moving beyond analysis to the practicalities of implementation, it is not clear how our scheme in Chapter 4, though distributed, can find its way into an actual implementation. An immediate impediment is estimating the parameters of the utility model. It might help to simplify the utility function, but this might be accompanied by a loss of accuracy. The incorporation of the dynamic aspects of fading is lacking in our model and the subsequent analysis. While this is consistent with the "one-shot event" philosophy that is pervasive in power control literature [15], we believe that the nature of a dynamic fading channel will have a significant impact on algorithm convergence and model parameter estimation. These intriguing problems are natural extensions of the research in this thesis. As a more immediate extension, it is possible to vary penalty function parameters as a function of some promised QoS class for each link. While seemingly simple to implement, the results would nevertheless be interesting.

A final thought - we believe that the last word in game-theoretic applications in wireless communications has not been said. At the wireless frontier there awaits a vast and growing collection of problems that have not been conclusively solved. We identify two fascinating areas - ad-hoc networks [79] and software radios [80] - that will ensure the vitality of wireless system engineering in the future. Ad-hoc networks do not have a fixed infrastructure, might have a constantly evolving network graph that is induced by node mobility, and may consist of dissimilar nodes with limited power and conflicting QoS objectives. In this sense, these networks are completely distributed and tasks such as network-formation, power control, and routing must be accomplished by each node in accordance with its objectives. Software radios are fully programmable radios that support several modes (frequencies, standards, etc.) of operation. The multi-mode 3G phones that are entering markets today are, in fact, rudimentary software radios. Undoubtedly, further developments will endow these devices with a mind-boggling complement of technologies and features. Software radios are likely to be characterized by their dynamic, real-time reconfigurability and the software implementation of a majority of their features. The RRM of networks of software radios will be complicated by the degree of heterogeneity introduced by having several such radios in a network. It might not be too far-fetched to suggest that software radios might be involved in the formation of ad-hoc networks. Established techniques for studying the comparatively bland fixed infrastructure systems, such as cellular networks, will not work without modification. At the same time, this is an excellent reason to consider game-theoretic techniques for this purpose. Several promising game-theoretic tools already exist. For example, the concept of a potential game [81] was unutilized in this thesis. Finite potential games are known to have NE in their pure strategies. These games have, to date, received little attention in the wireless literature [82]. Finding the right tools for the problems at hand is a genuine challenge confronting researchers adopting the gametheoretic approach. We speculate that the challenge of the future will be to unlock the potential of game-theoretic techniques to arrive at meaningful solutions to wireless engineering problems. 


\section{References}

[1] Ovum, "Next Generation Networks: Regional Variations," Ovum Marketing Research Report, 2003.

[2] iGilliot Research, "3G Report: An Overview of Implementation Status - A Market Brief," April 2003.

[3] Parry, R., "Overlooking 3G," IEEE Potentials, Oct./Nov. 2002, pp. 6-9.

[4] Zander, J., and Kim, S.L., Radio Resource Management for Wireless Networks, Artech House Publishers, Norwood, MA, 2001.

[5] Rappaport, T.S., Wireless Communications: Principles and Practice, $2^{\text {nd }}$ ed., Prentice-Hall, Upper Saddle River, NJ, 2002.

[6] Halonen, T., et al. (Eds.), GSM, GPRS and EDGE Performance: Evolution towards $3 G / U M T S, 2^{\text {nd }}$ ed., John Wiley and Sons, Chichester, 2003.

[7] Holma, H., Toskala, A., (Eds.), WCDMA for UMTS: Radio Access for Third Generation Mobile Communications, $2^{\text {nd }}$ ed., John Wiley and Sons, Hoboken, NJ, 2002.

[8] Axelby, G., Osborne, E., "Transmitter power control in two-way communications system," IEEE Transactions on Communications, Volume: 8, Issue: 1, Mar. 1960, pp. 48-56.

[9] Monk, A.M., Milstein, L.B., "Open-loop power control error on a land mobile satellite link," Third Annual International Conference on Universal Personal Communications, Sept.-Oct. 1994, pp. 205212.

[10] Lee, W.C.Y., "Power control in CDMA," Proceedings of the $41^{\text {st }}$ IEEE Vehicular Technology Conference, May 1991, pp. 77-80.

[11] Peterson, R.L., Ziemer, R.E., Borth, D.E., Introduction to Spread-Spectrum Communications, PrenticeHall, Englewood Cliffs, NJ, 1995.

[12] Sampath, A., Sarath Kumar, P., Holtzman, J.M., "On setting reverse link target SIR in a CDMA system," Proceedings of the $47^{\text {th }}$ IEEE Vehicular Technology Conference, May 1997, pp. 929-933.

[13]Blom, J., "Power Control in Cellular Radio Systems," Licentiate Thesis, Linkopings University, Sweden, June 1998. Available: http://www.control.isy.liu.se.

[14]Fujii, T., Sakamoto, M., "Reduction of co-channel interference in cellular systems by intrazone channel re-assignment and adaptive transmitter power control," Proceedings of the $38^{\text {th }}$ IEEE Vehicular Technology Conference, Jun. 1988, pp. 668-672.

[15]Zander, J., "Performance of optimum transmitter power control in cellular radio systems," IEEE Transactions on Vehicular Technology, Volume: 41, Issue: 1, Feb. 1992, pp. 57-62.

[16] Kreyszig, E., Advanced Engineering Mathematics, $8^{\text {th }}$ ed., John Wiley and Sons, New York, 2001.

[17]Zander, J., "Distributed co-channel interference control in cellular radio systems," IEEE Transactions on Vehicular Technology, Volume: 41, Issue: 3, Aug. 1992, pp. 305-311.

[18] Foschini, G.J. and Miljanic, Z., "A simple distributed autonomous power control algorithm and its convergence," IEEE Transactions on Vehicular Technology, Volume: 42, Issue: 4, Nov. 1993, pp.641646.

[19] Mitra D., "An asynchronous distributed algorithm for power control in cellular radio systems," Proceedings of $4^{\text {th }}$ WINLAB Workshop on $3 G$ Wireless Information Networks, Oct. 1993, pp. 249-257.

[20] Grandhi, S.A. et al., "Constrained power control," Wireless Personal Communications, Volume: 1, Issue: 4, 1995, pp. 257-270. 
[21] Berggren, F., "Power Control, Transmission Rate Control and Scheduling in Cellular Radio Systems," Licentiate of Technology dissertation, Royal Institute of Technology (KTH), Sweden, May 2001.

[22] Qiu, X., and Chawla, K., "On the performance of adaptive modulation in cellular systems," IEEE Transactions on Communications, Volume: 47, Issue: 6, Jun. 1999, pp. 884-895.

[23] Bazaraa, M.S., et al., Non-Linear Programming - Theory and Algorithms, $2^{\text {nd }}$ ed., John Wiley and Sons, New York, 1993.

[24] Wu, Q., "Optimum transmitter power control in cellular systems with heterogeneous SIR thresholds," IEEE Transactions on Vehicular Technology, Volume: 49, Issue: 4, Jul. 2000, pp. 1424-1429.

[25] Kim, S.L. et al., "Combined power control and transmission rate selection in cellular networks," Proceedings of the 50th IEEE Vehicular Technology Conference, Sept. 1999, pp. 1653-1657.

[26] Spiegel, M., Wrede, R., Schaum's Outline of Theory and Problems of Advanced Calculus, $2^{\text {nd }}$ ed., McGraw-Hill, New York, 2002.

[27] Soleimanipour, M., et al., "Optimal resource management in wireless multimedia wideband CDMA systems," IEEE Transactions on Mobile Computing, Volume: 1, Issue: 2, Apr.-Jun. 2002, pp. 143-160.

[28] Oh, S.J., et al, "Distributed power control and spreading gain allocation in CDMA data networks," Proceedings of IEEE Infocom, Mar. 2000, pp. 379-385.

[29] Leung, K.K., et al., "Integrated link adaptation and power control to improve error and throughput performance in broadband wireless packet networks," IEEE Transactions on Wireless Communications, Volume: 1, Issue: 4, Oct. 2002, pp. 619-629.

[30] Sampath, A., et al., "Power control and resource management for a multimedia CDMA wireless system," Proceedings of IEEE PIMRC, Sept. 1995, pp. 21-25.

[31] Osborne, M., Rubinstein, A., A Course in Game Theory, MIT Press, Cambridge, MA, 1994.

[32] Luce, R.D., Raiffa, H., Games and Decisions, John Wiley and Sons, New York, 1957.

[33] Dutta, P.K., Strategies and Games, MIT Press, Cambridge, MA, 1999.

[34] Von Neumann, J., and Morgenstern, O., Theory of Games and Economic Behavior, Princeton Univ. Press, Princeton, NJ, 1944.

[35] Nash, J.F. "Equilibrium points in $n$-person games," Proceedings of the National Academy of Science, Volume: 36, 1950, pp. 48-49.

[36] Harsanyi, J., "Games with incomplete information played by 'Bayesian' players: Parts I, II and III," Management Science, Volume: 14, 1967-68, pp. 159-182, 320-334, and 486-502.

[37] Selten, R., "Spieltheoretische Behandlung eines Oligopolmodells mit Nachfrageträgheit," Zeitschrift für die gesamte Staatswissenschaft, Volume: 121, 1965, pp. 301-324 and 667-689.

[38] Kakutani, S., "A generalization of Brouwer's fixed point theorem," Duke Mathematical Journal, Volume: 8, 1941, pp. 457-459.

[39] Debreu, G., "A social equilibrium existence theorem," Proceedings of the National Academy of Science, Volume: 38, 1952, pp. 886-893.

[40]Fan, K., "Fixed point and minima theorems in locally convex topological linear spaces," National Academy of Science, Volume: 38, 1952, pp. 121-126.

[41] Glicksberg, I.L., "A further generalization of the Kakutani fixed point theorem with application to Nash equilibrium points," Proceedings of the American Mathematical Society, Volume: 3, 1952, pp. 170-174.

[42] MacKenzie, A.B., Wicker S.B., "Game theory in communications: motivation, explanation, and application to power control," IEEE GlobeCom 2001, Nov. 2001, pp. 821-826. 
[43] LaValle, S.M., Hutchinson, S., "Game theory as a unifying structure for a variety of robot tasks," Proceedings of the 1993 IEEE International Symposium on Intelligent Control, Aug. 1993, pp. 429434.

[44]La, R.J., Anantharam, V., "Optimal routing control: game theoretic approach," Proceedings of the 36th IEEE Conference on Decision and Control, Dec. 1997, pp. 2910-2915.

[45] Bartal, Y., Rosen, A., "The distributed k-server problem - a competitive distributed translator for kserver algorithms," Proceedings of the 33rd Annual Symposium on Foundations of Computer Science, Oct. 1992, pp. 344-353.

[46] Maheswaran, R.T., Basar, T., "Multi-user flow control as a Nash game: performance of various algorithms," Proceedings of the 37th IEEE Conference on Decision and Control, Dec. 1998, pp. 10901095.

[47]Dziong, Z., Mason, L.G., "Fair-efficient call admission control policies for broadband networks-a game theoretic framework," IEEE/ACM Transactions on Networking, Volume: 4, Issue: 1, Feb. 1996, pp. 123-136.

[48] MacKenzie, A.B., Wicker, S.B., "Selfish users in Aloha: a game-theoretic approach," Proceedings of the 54th IEEE Vehicular Technology Conference, Oct. 2001, pp. 1354-1357.

[49] Shah, V., Mandayam, N.B., and Goodman, D.J., "Power control for wireless data based on utility and pricing," Proceedings of IEEE PIMRC, Sept. 1998, pp.1427-1432.

[50] Goodman, D.J, and Mandayam, N.B., "Power control for wireless data," IEEE Personal Communications Magazine, Volume: 7, Apr. 2000, pp. 48-54.

[51] Saraydar, C.U., Mandayam, N.B., Goodman, D.J., "Efficient power control via pricing in wireless data networks," IEEE Transactions on Communications, Volume: 50, Issue: 2, Feb. 2002, pp. 291-303.

[52] Yates, R.D., "A framework for uplink power control in cellular radio systems," IEEE Journal on Selected Areas in Communications, Volume: 13, Issue: 7, Sept. 1995, pp. 1341-1347.

[53] Saraydar, C.U., Mandayam, N.B., Goodman, D.J., "Pareto efficiency of pricing-based power control in wireless data networks," IEEE Wireless Communications and Networking Conference, Sept. 1999, pp. 231-235.

[54] Cocchi, R., Estrin, D., "Pricing in computer networks: motivation, formulation and example," IEEE/ ACM Transactions on Networking, Volume: 1, Issue: 6, Dec. 1993, pp. 614-627.

[55] Topkis, D. M., Supermodularity and Complementarity, Princeton Univ. Press, Princeton, NJ, 1998.

[56]Liu, X., Zhou, S., Xibin, X., “Optimal linear pricing in power control game," IEEE International Conference on Communications, Circuits and Systems and West Sino Expositions, Jun.-Jul. 2002, pp. 386-390.

[57] Saraydar, C.U., Mandayam, N.B., Goodman, D.J., "Pricing and power control in a multi-cell wireless data network," IEEE Journal on Selected Areas Communications, Volume: 19, Issue: 10, Oct. 2001, pp. 1883-1892.

[58] Sung, C.W., Wong, W.S., "A non-cooperative power control game for multirate CDMA data networks," IEEE Transactions on Wireless Communications, Volume: 2, Issue: 1, Jan. 2003, pp. 186193.

[59] Cover, T.M., Thomas, J., Elements of Information Theory, John Wiley and Sons, New York, 1991.

[60] Alpcan, T., Basar, T., Srikant, R., Altman, E., "CDMA uplink power control as a non-cooperative game," Proceedings of the 40th IEEE Conference on Decision and Control, Dec. 2001, pp. 197-202.

[61] Yin, Z., Xie, J., Lv, P., "An economic model for power control in wireless CDMA data networks," Proceedings of International Conference on Communication Technology, Apr. 2003, pp. 835-838.

[62] Rodriguez, V., "Robust modeling and analysis for wireless data resource management," IEEE Wireless Communications and Networking Conference, Mar. 2003, pp. 717-722. 
[63] Xiao, M., Shroff, N.B., Chong, E.K.P., "A utility-based power-control scheme in wireless cellular systems," IEEE/ACM Transactions on Networking, Volume: 11, Issue: 2, Apr. 2003, pp. 210-221.

[64] Yu, W., Ginis, G., Cioffi, J.M., "Distributed multiuser power control for digital subscriber lines," IEEE Journal on Selected Areas in Communications, Volume: 20, Issue: 5, Jun. 2002, pp. 1105-1115.

[65] Feng, N., Mandayam, N. B., and Goodman, D. J. , "Joint power and rate optimization for wireless data services based on utility functions," Proceedings of CISS, Mar. 1999, pp. 109-113.

[66] Hayes, J. F., "Adaptive feedback communications," IEEE Transactions on Communications Technology, Volume: COM-16, Feb. 1968, pp. 29-34.

[67] Cavers, J.K., "Variable-rate transmission for Rayleigh fading channels," IEEE Transactions on Communications, Volume: COM-20, Feb. 1972, pp. 15-22.

[68] Alamouti, S.M., and Kallel, S., "Adaptive trellis-coded multiple-phase-shift keying for Rayleigh fading channels," IEEE Transactions on Communications, Volume: 42, Issue: 6, Jun. 1994, pp. 2305-2314.

[69] Webb, W.T., and Steele, R., "Variable rate QAM for mobile radio," IEEE Transactions on Communications, Volume: 43, Issue: 7, Jul. 1995, pp. 2223-2230.

[70] Goldsmith, A., and Chua, S.-G., "Adaptive coded modulation for fading channels," IEEE Transactions on Communications, Volume: 46, Issue: 5, May 1998, pp. 595-602.

[71] Balachandran, K., Kadaba, S.R., Nanda, S., "Channel quality estimation and rate adaptation for cellular mobile radio," IEEE Journal on Selected Areas in Communications, Volume: 17, Issue: 7, July 1999, pp. 1244-1256.

[72] Ginde, S., Neel, J., Buehrer, R.M., "A game-theoretic analysis of joint link adaptation and distributed power control in GPRS," Proceedings of the 58th IEEE Vehicular Technology Conference, Oct. 2003, pp. $732-736$.

[73] Nader, G., "Radio Link Performance of Third Generation (3G) Technologies for Wireless Networks," Master of Science Thesis, Virginia Polytechnic Institute and State University, 2002.

[74] Polak, E., Computational Methods in Optimization - A Unified Approach, Academic Press, 1971.

[75] Molkdar, D. et al. "An overview of EGPRS: the packet component of EDGE," Electronics and Communications Engineering Journal, Volume: 14, Issue: 1, Feb. 2002, pp. 21-38.

[76] Gantmacher F.R., The Theory of Matrices v2., Chelsea, New York, 1959.

[77] Varga, R.S., Matrix Iterative Analysis, Prentice-Hall, Englewood Cliffs, NJ, 1962.

[78] Bazaraa M.S., Jarvis J.J., and Sherali H.D., Linear Programming and Network Flows, $2^{\text {nd }}$ ed., Wiley, New York, 1990.

[79] Perkins, C.E. (Ed.), Ad hoc Networking, Addison Wesley, Boston, 2001.

[80] Reed, J. H., Software Radio - A Modern Approach to Radio Engineering, Prentice-Hall, Upper Saddle River, NJ, 2002.

[81] Voorneveld, M., "Potential Games and Interactive Decisions with Multiple Criteria," Ph.D. dissertation, Tilburg University, The Netherlands, 1999.

[82] Neel, J., Reed, J.H., Gilles, R.P., "The role of game theory in the analysis of software radio networks," SDR Forum Technical Conference, November 2002. [Online]. Available: http://www.mprg.org/people/gametheory/files/neel_sdr_forum.pdf. 


\section{Vita}

Samir V. Ginde received his Master of Science degree in Electrical Engineering (EE) from Virginia Tech in May 2004. From May 2002 to May 2004 Samir worked as a Graduate Research Assistant to Dr. R. Michael Buehrer of the Mobile and Portable Radio Research Group (MPRG). His thesis research involved applying game-theoretic techniques to link adaptation. His research interests lie in the areas of radio resource management for wireless networks, CDMA and application of game theory to wireless systems. In the summer of 2004, Samir began a new life in industry; he has accepted a job offer to work at Qualcomm Inc. in San Diego, CA as a CDMA systems engineer. Samir grew up in Mumbai (formerly Bombay), India, and earned his Bachelor of Engineering in Electronics Engineering (with distinction) from Mumbai University in 2001. Outside his profession, Samir is passionate about music, and sport - excelling at soccer. He is a member of the Institute of Electrical and Electronics Engineers (IEEE).

\section{Publications}

Ginde, S., Neel, J., Buehrer, R.M., "A game-theoretic analysis of joint link adaptation and distributed power control in GPRS," Proceedings of the 58th IEEE Vehicular Technology Conference, Oct. 2003, pp. 732-736. 